
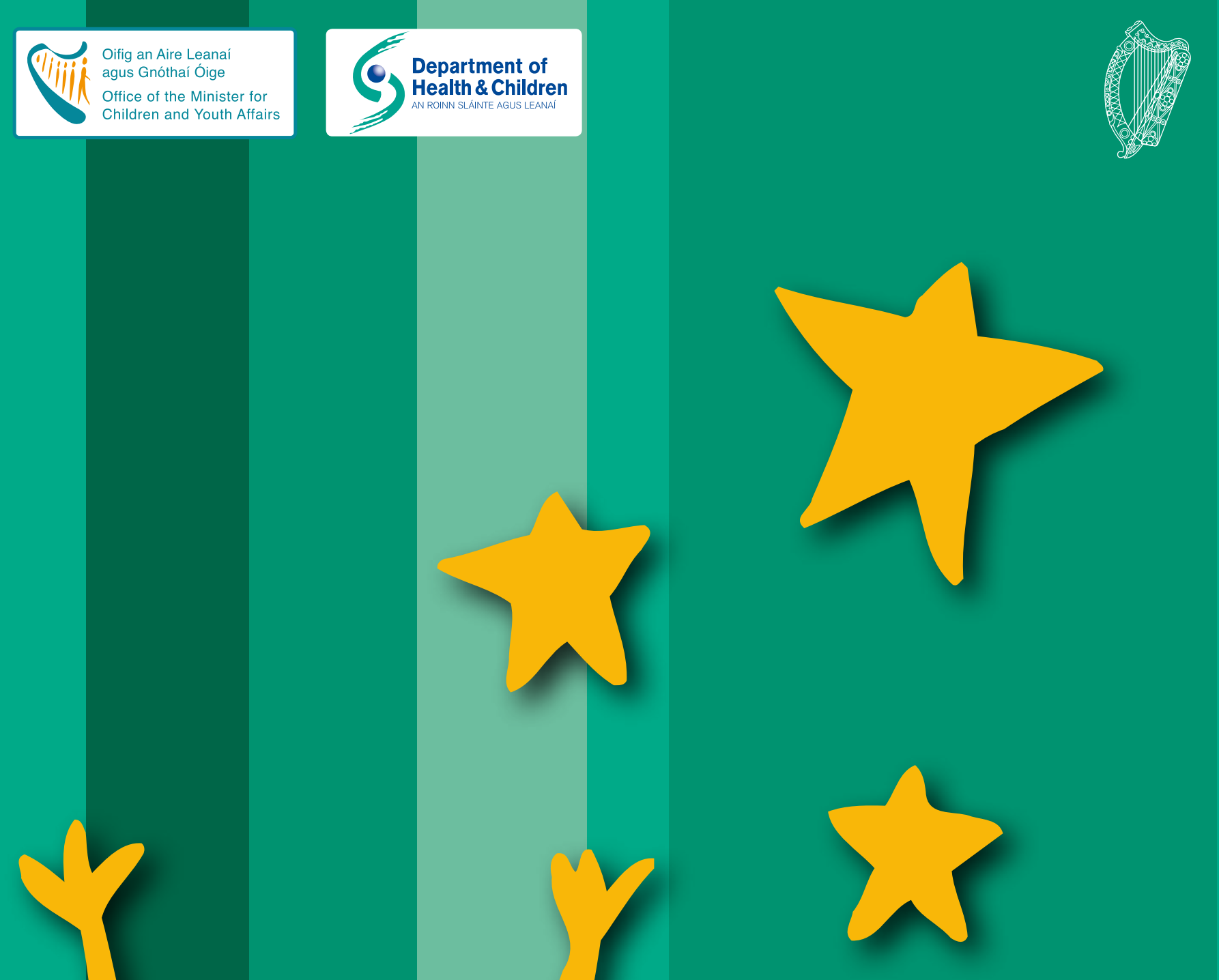

\title{
Fthical Review
} and Children's Research in Ireland

The National Children's Strategy Research Series 



\section{Ethical Review and Children's Research in Ireland}


The authors of this report are:

Heike Felzmann, Jane Sixsmith, Siobhan O'Higgins, Sorcha Ni Chonnachtaigh and Saoirse Nic Gabhainn,

Centre of Bioethical Research and Analysis

(www.nuigalway.ie/cobra) and Health Promotion

Research Centre, National University of Ireland, Galway

(www.nuigalway.ie/hprc).

Copyright $\odot$ Minister for Health and Children, 2010

Office of the Minister for Children and Youth Affairs

Department of Health and Children

Hawkins House

Hawkins Street

Dublin 2

Tel: $+353(0) 16354000$

Fax: $+353(0) 16743223$

E-mail: omc@health.gov.ie

Web: www.omc.gov.ie

Published by The Stationery Office, Dublin

ISBN 978-1-4064-2495-9

The views expressed in this report are those of the authors and not necessarily those of the Office of the Minister for Children and Youth Affairs.

All rights reserved. No part of this publication may be reproduced, stored in a retrieval system, or transmitted, in any form or by any means, electronic, mechanical, photocopying, recording or otherwise, without the prior permission in writing of the copyright holder.

For rights of translation or reproduction, applications should be made to the Head of Communications, Office of the Minister for Children and Youth Affairs, Hawkins House, Hawkins Street, Dublin 2, Ireland. 


\section{Contents}

\section{Acknowledgements}

Acronyms used

1. Introduction 1

Research aims and objectives $\quad \begin{aligned} & 1 \\ & \text { Structure }\end{aligned}$

Structure of report

2. Methodology

Overall design

Literature/documentary review

Stakeholders' perspectives

Research Ethics Committees

Children's Researchers

Children

Parents

Health Service Executive (HSE) Consultation

Data presentation

Ethical approval

3. Research Ethics Governance: The international context

A general introduction to Research Ethics Committees

International models

Canada

USA

United Kingdom

Denmark

Australia

New Zealand

Issues for current models

Between diversity and standardisation

Governing different research sectors and research fields

The REC bureaucracy: Costs and burdens

Main concerns

\section{Research Ethics Committees: Structures, functions}

\section{and processes}

Irish Research Ethics Committees - The landscape

Healthcare RECs and Clinical Trials RECs

Academic RECs

Other committees

Composition of international RECs

Composition of Irish RECs

Stakeholders' perspectives on Irish RECs

Specific REC membership

Remit, workload and functions of international RECs

Remit and workload of Irish RECs 
Ethics Review Process

Standard operating procedures and application materials

Stakeholders' perspectives on standard operating procedures and application procedures

Review process and results

Stakeholders' perspectives on review processes and results

Stakeholders' perspectives on record-keeping and monitoring

\section{Ethical issues specific to research methodology} and research settings

Ethics, methodology and Social Science and Humanities research

Ethical issues in relation to methodology and settings for children's research

Stakeholders' perspectives on ethical issues specific to children's research methodology and settings

Main concerns

\section{Ethical issues in children's research}

Informed consent in research ethics

General concerns

Navigating informed consent and assent in children's research

Stakeholders' perspectives on informed consent

Confidentiality in research

Confidentiality in children's research

Stakeholders' perspectives on confidentiality

Risk and benefit

Generic concerns regarding risk, burden and benefit

Risk and benefit in research with children

Stakeholders' perspectives on risk and benefit in children's research Main concerns

\section{The Ethics of Children's Research in Ireland}

The tensions

Stakeholders' perspectives for the future

Conclusion

Recommendations

1. Creation of central research ethics resource

2. Comprehensive governance system and national standardisation

3. Creation of suitable structures for the review of children's research

4. Meeting specific information needs

5. Involving children in the research process

\section{References}

\section{Appendices}

Appendix 1: Survey Questionnaire Instrument

Appendix 2: List of Irish Research Ethics Committees for Human Subject Research Appendix 3: Semi-structured Interview Schedule for Chairperson/Discussion topics for Administrators 


\section{Acknowledgements}

The Research Team would like to acknowledge the assistance and support of all those who participated in this research, including the children, parents, researchers, Research Ethics Committee (REC) members, chairpersons and administrators who took time out of their busy schedules to participate in the research. In addition, we would like to thank the Office of the Minister for Children and Youth Affairs, the NUI Galway librarians, and the directors, administrators and coordinators of those research units that assisted us in conducting the focus groups with researchers.

We also fully acknowledge the collaboration with the HSE research team and the staff who worked with us to avoid unnecessary overlap and duplication in data collection, specifically in the case of questionnaire data from RECs.

The Principal Investigator for this research is Dr. Heike Schmidt-Felzmann of the Department of Philosophy, NUI Galway, who worked directly with post-graduate researchers Sorcha Ni Chonnachtaigh and Marion Ward. Staff of the Health Promotion Research Centre were also involved in proposal development, research management, data collection and analysis, report writing and editing, specifically Dr. Jane Sixsmith, Ms. Siobhan O'Higgins, Ms. Aine O'Connell and Dr. Saoirse Nic Gabhainn. Ms. Anne McMahon also worked on the data analysis of the interviews with REC chairpersons and administrators.

Heike Felzmann, Jane Sixsmith, Siobhan 0'Higgins, Sorcha Ni Chonnachtaigh and Saoirse Nic Gabhainn Centre of Bioethical Research and Analysis, Health Promotion Research Centre, National University of Ireland, Galway 


\section{Acronyms used}

ACSTI

AHEC

Advisory Council for Science, Technology and Innovation (Ireland)

AREC

Australian Health Ethics Committee

CIHR

Association of Research Ethics Committees (UK)

COREC

Canadian Institutes of Health Research

$\mathrm{DoH}$

Central Office for Research Ethics Committees (UK)

DoHC

Department of Health (UK)

ESRC

FWA

HHS

HRC

HREC

ICB

ICRIN

IRAS

IRB

NEAC

NHMRC

Department of Health and Children (Ireland)

Economic and Social Research Council (UK)

Federal Wide Assurance (USA)

US Department of Health and Human Services (USA)

Health Research Council (New Zealand)

Human Research Ethics Committee

Irish Council for Bioethics

Irish Clinical Research Infrastructure Network

Integrated Research Application System (UK)

Institutional Review Board (USA), equivalent to REC

National Ethics Advisory Committee (New Zealand)

NACHDSSE

NPSA

NRES

NSERC

NZHDEC

OHRP

PRE

REB

REC

SSHRC

SSHWC

TCPS

UNCRC

National Health and Medical Research Council (Australia)

National Advisory Committee on Health and Disability Support Services Ethics

(New Zealand)

National Patient Safety Authority (UK)

National Research Ethics Service (UK)

Natural Sciences and Engineering Research Council (Canada)

New Zealand Health and Disability Ethics Committees

Office for Human Research Protections (USA)

Interagency Panel on Research Ethics (Canada)

Research Ethics Board

Research Ethics Committee

Social Sciences and Humanities Research Council (Canada)

Social Sciences and Humanities Research Ethics Special Working Committee (Canada)

Tri-Council Policy Statement (Canada)

United Nations Convention on the Rights of the Child

\section{Research stakeholders}

$\mathrm{AC}$

$\mathrm{HC}$

A

CM

HSE

CR

$\mathrm{P}$

C
Chair of Academic REC

Chair of Hospital REC

REC Administrator

Member of REC

HSE Consultation Day

Children's researcher

Parent

Child 
1 INTRODUCTION 
A substantial increase in research into the lives of children has been experienced over the last 20 years in many countries, including Ireland. This increase is not only in the amount of research undertaken, but also in the multidisciplinary range of such research. Research with children involves working with potentially vulnerable participants, who not only require specific protection but also have the capacity for independent agency that necessitates respect from researchers.

With the increase in research, there has been a related increase in awareness by the research community of the need to ensure that all research with children is carried out to the highest ethical standards. Until recently, ethical review of research was left to the discretion of the researchers, who were generally considered sufficiently trustworthy to safeguard their participants' rights and well-being by virtue of their education and professional standing. Over the last two decades, however, it has become more common to convene Research Ethics Committees to review research proposals with the goal of ensuring that ethical requirements are fulfilled and to provide ethical oversight to researchers. This has resulted in an increase in the number of Research Ethics Committees operating worldwide, including in Ireland.

In relation to children's research, ethical review attempts to ensure that research projects have provisions in place that will enable researchers to do justice to the duties of both protection and respect of child participants. However, a variety of problematic issues have been highlighted. Research with children into the ethics of children's research has identified tensions between balancing risk and benefit, on the one hand, and autonomy, competence and parental authority, on the other. The reliability and validity of review by Research Ethics Committee has also been brought into question: not only are requirements vastly different between countries (Hearnshaw, 2004), but there is ample evidence to show that decision-making by different Research Ethics Committees in the same country can differ significantly (Garfield, 1995), even when they are governed by the same authority (Angell et al, 2006). In order to counter this variability, it has been argued that increased regulation needs to be put in place and this has happened in some countries.

Research ethics review in Ireland is a more recent development and is less regulated than in other countries. It is mostly influenced by the guidelines on Research Ethics Committees of the Irish Council for Bioethics (ICB, 2004). Overall, few national studies on the functioning and remit of Research Ethics Committees have been conducted and large-scale studies of review of research on children have been particularly rare (Shah et al, 2004). Thus, the evolution of Irish research ethics review in a relatively unregulated environment, coupled with the increase in multidisciplinary research on children's lives, warrants investigation.

\section{Research aims and objectives}

The main aim of this research is to provide an overview of the current mechanisms for applying for and achieving ethics approval for studies being undertaken with children in Ireland.

To meet this aim, the objectives of the research are:

- to undertake a comprehensive literature review on the structures for seeking and achieving ethics approval internationally;

- to examine the extent to which formal approval for research being undertaken with children in Ireland is sought and achieved;

- to identify the number, composition and remit of Research Ethics Committees operating in Ireland which provide a mechanism for ethics approval for research with children;

- to provide information on the number of meetings held annually by these Research Ethics Committees and the waiting times for ethics approval for each committee;

- to identify key issues arising in respect of ethics approval for children's research according to different stakeholders;

- to make recommendations about the structures for seeking and achieving ethics approval for children's research in Ireland. 


\section{Structure of report}

This research represents two distinctly separate pieces of work carried out: a literature review and an exploration with stakeholders on the ethical review process for children's research. The report is structured as follows:

- Chapter 2 presents the research methods used to provide the context for the subsequent chapters, which integrate the research literature with empirical data gathered.

- Chapter 3 presents a review of research ethics governance in various countries, with examples from a range of national governance structures and an exploration of the issues and tensions in each.

- Chapter 4 describes the structures and processes used in Ireland for ethical review by Research Ethics Committees.

- Chapter 5 reviews the ethical issues specific to research methodologies and research settings.

- Chapter 6 identifies specific issues of concern in children's research, such as consent, confidentiality, and risk/burden and benefit.

- Chapter 7 examines the current tensions in children's research in Ireland and considers the possible future from the perspectives of the stakeholders participating in this study. Finally, a number of recommendations are made to negotiate developments in the future. 


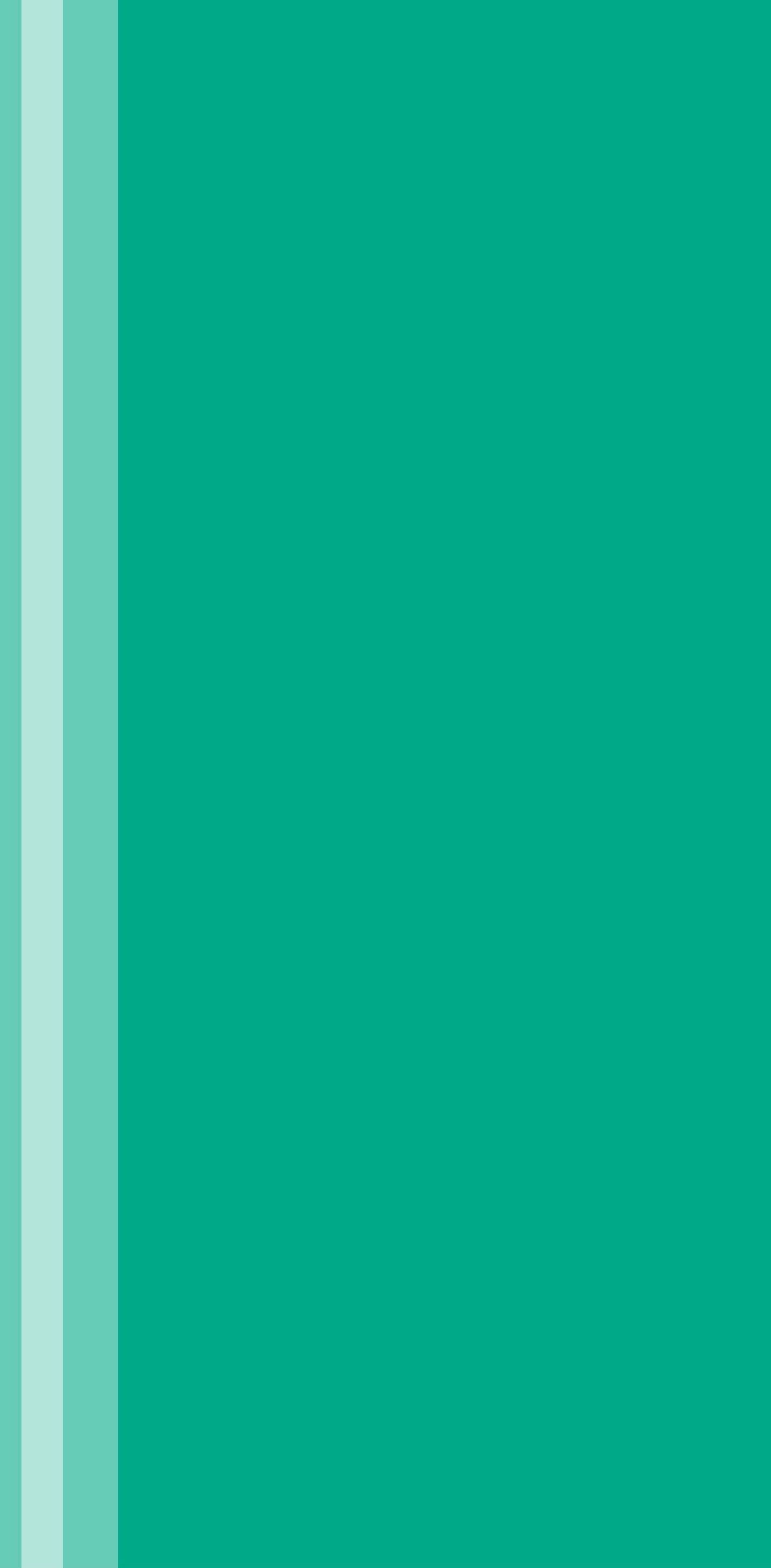


METHODOLOGY 


\section{Overall design}

The range of requirements as stipulated by the aims and objectives of this research (see Chapter 1) dictates a multi-method approach to research design (Robson, 2002). The majority of the data collected for this study was qualitative in nature, with some quantitative data also gathered and collated. For clarity, the methods employed are presented for the two main areas - the literature/ documentary review and the stakeholders' perspectives.

\section{Literature/documentary review}

The literature review draws on studies in the fields of philosophy, medicine, social science and the humanities in order to identify ethical issues in research with children, international good practice regarding review processes, institutional structures and analyses of challenges encountered in establishing ethics review procedures. An area of particular focus is the Irish situation regarding ethics review and the conduct of children's research.

The search strategy employed to identify relevant material comprised a number of approaches. For the literature review, a search of specific databases was undertaken, including Medline, Pubmed, Social Science Citation Index, Science Direct and Philosophers' Index. Searches were restricted to the English language using the primary keywords 'research ethics', 'research ethics committee', 'IRB', 'REB' and 'REC', in combination with 'children', 'paediatric', 'adolescents', including relevant variations of these terms. Additional search terms included core research ethics concepts like 'informed consent', 'assent', 'autonomy', 'competence', 'risk', 'benefit', 'harm' and 'confidentiality'.

Once relevant journals with a significant proportion of research ethics articles had been identified, an issue-by-issue search of their contents was conducted. In most cases, the search went back to the year 1995. However, in the case of the first specialised research ethics journal from the USA, IRB: Ethics and Research, all back issues to 1978 were considered in order to identify which topics were prominent in the debate at an early stage of Research Ethics Committee development and to consider them in relation to current debates.

Web-based searches, using Google and All the Web, were also conducted with three main purposes:

- identification of research ethics bodies internationally and their guidelines, documentation and supporting materials;

- identification of Irish Research Ethics Committees and research ethics guidelines;

- identification of international bodies and guidelines dedicated to children's research.

\section{Stakeholders' perspectives}

Stakeholders' perspectives were ascertained through both quantitative and qualitative approaches to data collection. A quantitative postal survey of identified Research Ethics Committees was undertaken, the aim of which was to gather information on their remit, procedures, specific practices and extent of applications relating to research with children (see Appendix 1). A variety of qualitative data collection techniques, including semi-structured interviews and focus groups, were also used to gather information from specific stakeholders (see Appendices 3-6). These comprised chairpersons, administrators and members of Research Ethics Committees, both academic and medically orientated (see Appendix 2), as well as children's researchers, parents and children.

\section{Research Ethics Committees}

In the process of undertaking this research, it became apparent that a review to identify Research Ethics Committees (RECS) and their processes of gaining research ethics approval was being undertaken by the Health Service Executive (HSE Research Ethics Committee Review Group, 2008). It was agreed that a limited collaborative approach to specific aspects of data collection in relation to members of RECs would be employed. This collaboration consisted of regular meetings and telephone conferences between the research teams of the HSE and the National University of 
Ireland (NUI), Galway; collaborative development and evaluation of the survey instrument; pooling of relevant contact details; input by the NUI Galway team into the planning and realisation of the HSE Consultation Day; and input into the writing and revision of the final HSE report - all under the lead of the HSE team. The stakeholder interviews and focus groups with chairpersons, administrators, researchers, parents and children were planned, conducted and analysed by the NUI Galway team alone, with the limited exception of the development of the chairpersons' interview guidelines which received feedback from the HSE team. (For conclusions of the HSE report, see Appendix 8.)

\section{Sample survey}

The survey sample frame was developed from a listing of operational Research Ethics Committees (RECs) provided by the Irish Council of Bioethics and was expanded to include additional committees identified through the literature/documentary review process. A total of 50 RECs were identified during the first stage of the research: 10 based in academic institutions and 27 hospital/medical-based (of which 13 assessed clinical trials), 7 in voluntary bodies and 6 in other organisations (see Appendix 2). A census sample frame of all RECs identified was used.

\section{Survey data collection}

A postal questionnaire was developed in conjunction with the HSE research team and reviewed by its steering committee; it was pilot-tested and minor changes were subsequently made (see Appendix 1). The administration, distribution and collation of the questionnaire data was undertaken by the HSE research team. Responses were received from 32 RECs in total, which are included in the analysis. No replies were received from the remaining 18 , resulting in a response rate of $64 \%$. The response rate was comparable for all types of committee. For this report, the data were analysed at the Health Promotion Research Centre in NUI Galway, using the computer package SPSS for Mac, Version 11.0.

During the qualitative data collection following the survey, 22 further RECs were identified, most of them sub-committees in academic institutions (see Appendix 2). Sub-committees had been initially excluded due to the expectation that each institution should only be represented by the REC that had the primary review function in the institution. However, it transpired that many of these subcommittees were functioning independently from each other and had considerable review loads. The remaining additional RECs included 2 previously not identified primary academic committees and 2 committees in the health and social care sector. One committee from the original Irish Council of Bioethics' list turned out to be inoperational due to falling under the remit of another committee.

\section{Semi-structured interviews}

In addition to the questionnaire survey, semi-structured telephone interviews with 26 chairpersons of RECs (14 of whom chaired academic committees and 12 chaired hospital/medical committees) and 8 administrators were undertaken. The semi-structured interview format was pilot-tested prior to field use and minor changes made (see Appendix 3). In the process of organising the semi-structured interviews, it was established that while the 18 RECs that did not respond to the survey were formally constituted, some lacked a named chairperson and/or administrator. In recruiting respondents for the semi-structured interviews, care was taken to include a balance between healthcare and academic committees, and to include those committees that had substantial experience with the review of children's research. All interviews undertaken were digitally recorded and transcribed verbatim. Analysis was undertaken through a template approach as described by Robson (2002).

Two focus groups were also undertaken with members of RECs and 4 individual interviews with REC members, two of whom were also children's researchers. The topic guide format was similar to that used in the semi-structured interviews with REC chairpersons and administrators (see above), although tailored to the perspective and responsibilities of REC members (see Appendix 4). As with the analysis process for the semi-structured interview data, the focus groups were digitally recorded and transcribed verbatim, with analysis being undertaken using Robson's (2002) template approach. 


\section{Children's Researchers}

\section{Sample}

Researchers of children in Ireland were identified through the literature/documentary review process described above, with web-based searches of organisations and institutions expressing an interest in research with children or on children's issues. In addition, the database 'Expertise Ireland' was used to identify academic researchers with an interest in children's research. Individual researchers and clusters of children's research were identified through this process.

\section{Data collection}

Focus groups were carried out with children's researchers based in three institutions (two academic and one medical) in Dublin, Cork and Galway. A total of 6 focus groups were undertaken. One other institution was approached, but declined to participate; two other institutions were willing to participate in a focus group, but scheduling turned out not to be feasible. A moderator and assistant moderator facilitated the focus groups, the topic guide for which was pilot-tested, with minor changes made (see Appendix 5). Data were digitally recorded and transcribed verbatim. Contemporaneous written notes were made by the assistant moderator at each focus group. Data were analysed using Robson's (2002) template approach.

In addition to the 6 focus groups, 8 individual interviews with children's researchers from 5 institutions were conducted, two of whom were also involved in a Research Ethics Committee.

\section{Children}

\section{Sample}

Children and young people were accessed through schools. The Principals of primary schools who had participated in a children's survey were contacted. Four schools agreed to participate. Active consent forms were sent to all the parents of students who had participated in a research study within those schools. In all the schools, the parents actively agreed for their children to participate in the research process. Students were asked by the researcher to provide separate assent for participation in the study (see Appendix 7). None of the students chose not to participate, but one student did not participate due to not returning the parental consent form. In total, 47 students aged 11-13 participated in the study.

\section{Data collection}

A participative workshop protocol was initially designed by the research team. The areas focused on were those consistently highlighted in the literature as important in relation to ethical issues for children's research - i.e. consent, confidentiality and risk/burden. Two full pilot workshops were organised in a post-primary and primary school where the students had participated in research during the previous year. The secondary school students were conceptualised as being an expert group to comment and inform the workshop protocol for the main study.

The resultant participative workshop protocol was divided into three parts. The first and last sections of the workshop involved the students filling in a table of responses to issues about confidentiality and risk/burden. They were then asked to move to specific areas of the room that represented 'Yes/No/Unsure' responses. A discussion was facilitated during which those in either the 'Yes' or the ' $\mathrm{No}$ ' areas tried to persuade those in the 'Unsure' area to move to join them. In so doing, the reasons for their responses were illuminated and discussed within the group. The middle section of the workshop was a facilitated discussion on the idea of consent.

The workshops were all recorded and notes taken by a co-facilitator. The data were subjected to content analysis. Simple counting techniques were also used, as advocated by Silverman (1993), to give a visual overview of the young people's responses to the issues raised. 


\section{Parents}

\section{Sample}

A list of 113 schools known to have participated in research was identified from the Department of Education and Science data file of schools and 20 primary schools were randomly selected from the list compiled. Fifteen of the 20 Principals from each of the 20 schools identified recruited a parent, 13 of whom participated in the study. Of those who participated, 8 were mothers and 5 were fathers of children that had taken part in previous research projects.

A semi-structured telephone interview was undertaken with each parent. The interview schedule was pilot-tested prior to field use (see Appendix 6). Each interview was digitally recorded and analysed using a template approach (Robson, 2002) consistent with that developed in previous aspects of data collection for this study.

\section{Health Service Executive (HSE) Consultation}

The HSE initiative Review of Research Ethics Committees and Processes in Republic of Ireland (2008) also included a Consultation Day, with invited members of Research Ethics Committees, researchers, patients' representatives, experts and policy-makers. The NUI Galway team was involved in the preparation and realisation of this Consultation Day and some dialogue recorded from this meeting is included in this report. (For the full HSE report, see www.hse.ie/eng/services/Publications/corporate/ etr/Review_of_Research_Ethics_Committees_and_Processes_in_Republic_of_Ireland.html.)

\section{Data presentation}

The data collected through the literature/documentary review and collation, along with that generated by the semi-structured interviews and focus groups, were integrated in the final stage of analysis and are presented together in this report. Quotes from various stakeholders are given throughout the text to illustrate specific aspects of the data; quotes are coded according to the stakeholder group to which the participant belonged, e.g. 'AC' for Chair of Academic REC (see Table 1). Quotes have been subjected to minimal editing in order to retain the tenor of the comments made.

Table 1: Legend for stakeholder groups

\begin{tabular}{|l|l|}
\hline Stakeholder group & Code \\
\hline Chair of Academic REC & AC \\
\hline Chair of Hospital REC & HC \\
\hline Administrator & A \\
\hline Member of REC & CM \\
\hline HSE Consultation Day & HSE \\
\hline Children's Researcher & CR \\
\hline Parent & P \\
\hline Child & C \\
\hline
\end{tabular}

\section{Ethical approval}

Approval for conducting this research was sought and granted from the Research Ethics Committee of the National University of Ireland, Galway. 


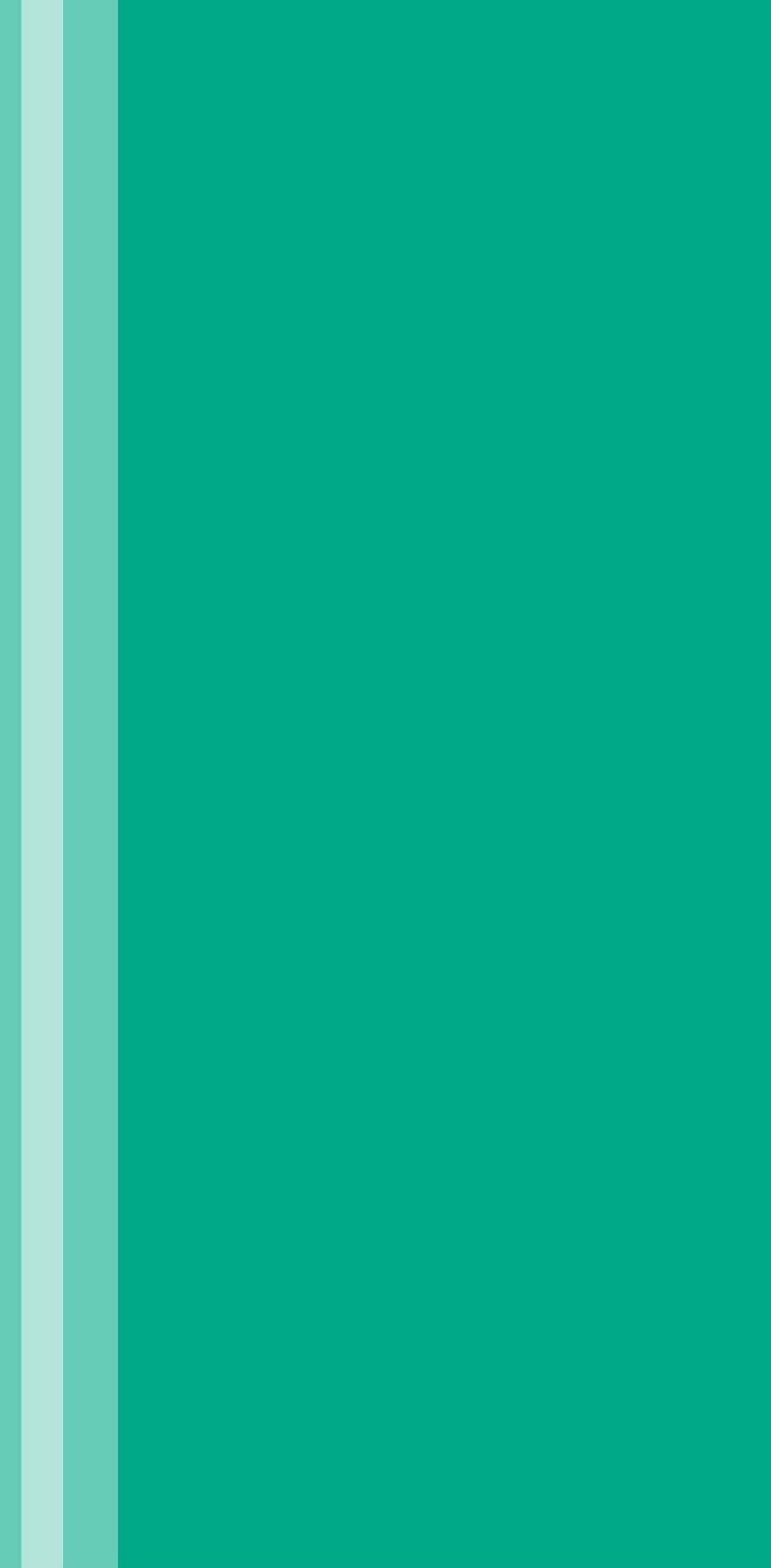




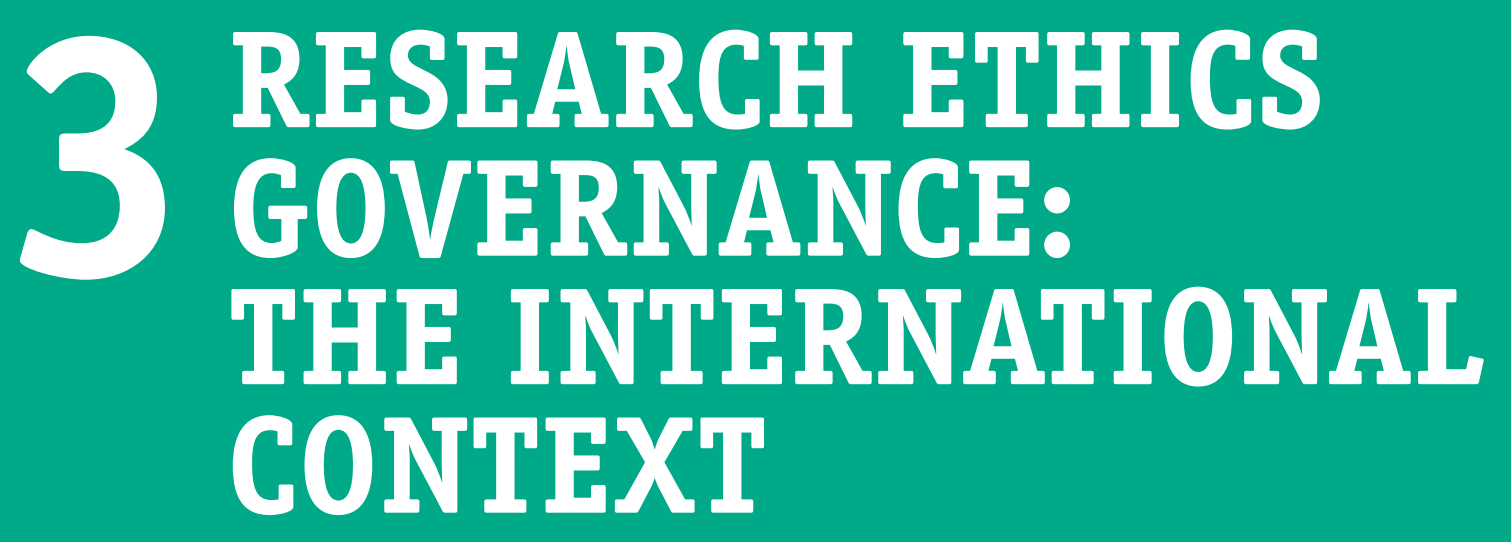




\section{A general introduction to Research Ethics Committees}

Research Ethics Committees are bodies whose primary function is to review the ethical quality of research proposals, rather than their scientific quality. The core ethical concern of such committees is the protection of research subjects from becoming enrolled in unethical research protocols. As the World Health Organization states in its Operational Guidelines for Ethics Committees that review Biomedical Research, the role of a Research Ethics Committee is 'to contribute to safeguarding the dignity, rights, safety and well-being of all actual or potential research participants' (WH0, 2000, p. 1).

Research Ethics Committees (RECS) are composed of a group of reviewers, as opposed to review by individuals. Since the early 1980s, they have become an increasingly prominent fixture of the research scene and are currently one of the core providers of ethical review of research with human and animal subjects. In different countries, alternative descriptions are used for these bodies, including 'Institutional Review Board' (IRB), especially for committees in the USA, or 'Research Ethics Board' (REB) for committees in Canada.

RECs are created at different levels and by different types of bodies or organisations. Accordingly, their remit can differ significantly, from research that takes place within a particular region or country, to research performed in individual organisations, like hospitals, or even in sub-units of organisations, like academic departments. Different systems internationally have found quite different solutions to the question of the organisation of their research ethics governance system. There seems to be currently no international consensus regarding the optimal structure of the governance system. In some jurisdictions, research ethics governance structures are very well developed. In many cases, modifications to existing governance systems were preceded by a process of extensive review and consultation, for example, in the UK by the Central Office for Research Ethics Committees (2004 and 2006) and the Department of Health (2005); and in New Zealand by the National Advisory Committee on Health and Disability Support Services Ethics (2003).

The main issues in the debate seem to be how to assess the advantages and disadvantages of centralised review systems, on the one hand, and institution-based systems of review, on the other, and also how to delineate the specific tasks that a governance structure needs to fulfil.

\section{International models}

Internationally, institution-based systems are still the norm; fully centralised systems are comparatively rare. Historically, most of the institution-based systems are the result of an initially uncoordinated development of RECs in those institutions that perceive a need for REC structures. Once these RECs have been set up, further developments in research ethics governance with a larger scope frequently follow. These include the introduction of binding norms or regulations for RECs within a jurisdiction or within a certain organisational framework. Centralised systems tend to arise as a result of further reform of an institution-based system, as one way to address concerns about accountability, common standards and quality assurance in REC review. As an introduction to approaches to research ethics governance, the following discussion presents a range of international models of research ethics governance.

\section{Canada}

The 'Tri-Council Policy Statement: Ethical Conduct for Research involving Humans' describes standards and procedures for governing research involving human subjects throughout Canada (CIHR et al, 1998/2005) and is currently in the process of being revised; the revised statement is expected to be published in December 2009 (see http://pre.ethics.gc.ca/eng/index). Canada has created and funded three agencies to promote, support and undertake research - the Canadian Institutes of Health Research (CIHR), the Natural Sciences and Engineering Research Council (NSERC) and the Social Sciences and Humanities Research Council (SSHRC). These agencies 
adopted the Tri-Council Policy (TCP) in August 1998 as a common research ethics policy for those conducting, participating in or reviewing human research in institutions funded by the CIHR, NSERC and SSHRC. These agencies will consider funding (or continued funding) only to individuals and institutions that certify that they comply with this research policy.

In addition, several Canadian Federal Government organisations, such as the National Research Council, the Canadian Space Agency, Health Canada and National Defence, have adopted the TCP. Some professional bodies and Provincial Government departments also use it. The Interagency Advisory Panel on Research Ethics (PRE) is a body of external experts, established in November 2001 by the CIHR, NSERC and SSHRC, to support the ongoing evolution and development of the TCP (see http://pre.ethics.gc.ca/eng/panel-group/about-apropos/reference).

The TCP provides a standardised ethical research policy, including detailed regulations regarding:

Ethics Review Boards (Article 1)

Informed Consent (Article 2)

Privacy and Confidentiality (Article 3)

Conflict of Interest (Article 4)

Inclusion (Article 5)

\author{
Aboriginal Peoples (Article 6) \\ Clinical Trials (Article 7) \\ Genetic Research (Article 8) \\ Gametes/Embryos/Foetuses (Article 9) \\ Human Tissue (Article 10)
}

The TCP does not include any separate section on children's research and the comments on children's research remain generally unchanged in the draft revision of the TCP. It does, however, mention issues relevant to children's research in different places throughout, including:

- informed consent and the role of trust and dependency for voluntariness in participation in research;

- involvement of additional parties in the informed consent process for participants who are not competent to consent;

- confidentiality and its limitation due to mandatory reporting laws;

- the danger of discrimination and the importance of research involving populations that are not fully competent to give consent (for example, children), but that could as a group benefit from research.

\section{USA}

The Office for Human Research Protections (OHRP) in the USA provides leadership to the system for protecting volunteers in research that is conducted or supported by the US Department of Health and Human Services (HHS). OHRP provides clarification and guidance to research institutions, develops educational programmes and materials, and promotes innovative approaches to enhancing human subject protections. Nearly 10,000 universities, hospitals and other research institutions in the USA and abroad have formal agreements with OHRP to comply with the regulations relating to the protection of human subjects. These are codified in the HHS's 2005 Code of Federal Regulations (CFR) in Titles 45 (Public Welfare) and 46 (Protection of Human Subjects), Subparts A-D (see www.hhs.gov/ohrp/humansubjects/guidance/45cfr46.htm).

In 1991, 14 other Federal departments and agencies joined the HHS in adopting a uniform set of rules for the protection of human subjects, called the Federal Policy for the Protection of Human Subjects (known as the 'Common Rule'). The HHS employs a system of Institutional Review Boards (IRB registration) or Independent Ethics Committees (IEC) and assurances. HHS regulations require institutions to commit to compliance with Regulation 45 CFR Part 46 before initiating participation in HHS-conducted or supported research involving human subjects. In essence, OHRP depends on institutional officials, committees, researchers and other agents of OHRP to comply with its assurance and the Federal Regulations.

All institutions engaged in human research (not exempt from the Federal Regulations), and conducted by any HHS agency, must be covered by an OHRP-approved assurance of compliance. The Federal Wide Assurance (FWA) is the only type of assurance accepted and approved by OHRP. Any Federal 
department or agencies that have signed on to the Common Rule (Regulation 45 CFR Part 46) and have accepted an OHRP-approved FWA must designate on the FWA the IRB/IEC(s) that will perform initial and continuing review of the research. Obtaining an approved assurance from OHRP is a two-step process: first, the IRB(s) designated under the assurance must be registered with OHRP and, second, an assurance application (FWA) must be completed.

Ethical requirements in children's research are stated in Regulation 45 CFR Part 46, Subpart D: 'Additional Protections for Children involved as Subjects in Research'. Subpart D provides additional protection for children participating in research beyond those that apply to fully competent adults, as follows (see www.hhs.gov/ohrp/humansubjects/guidance/45cfr46.htm\#subpartd and www.hhs.gov/ohrp/researchfaq.html):

1. The scope of exemption from IRB review is reduced for children's research, i.e. some research activities are exempt for adults, but need to be reviewed if children are participants (e.g. educational interviews and surveys).

2. A dual process of parental permission and child assent is required instead of obtaining informed consent only from the participant. Child assent involves active affirmative agreement, not just the absence of dissent. However, depending on the type of research and the maturity of the child participant, waivers of parental consent or child consent are possible under some exceptional circumstances.

3. The guidelines identify three types of children's research that can be approved, based especially on the risk and benefit profile of the research. These categories are more narrowly defined than those that can be approved for research with competent adults.

4. Under special circumstances, research can be approved even if it does not fit these categories, provided it is likely to lead to a significantly improved understanding, prevention or alleviation of an important problem for children's health or welfare.

5. Some additional conditions exist for research activities with children who are wards of the State or other agencies.

\section{United Kingdom}

National Health Service (NHS) Research Ethics Committees (RECs) have been established throughout the UK with the purpose of safeguarding the rights, dignity and welfare of people participating in research in the NHS. Potential research participants at NHS organisations in the UK will come under the protection of a REC. There are currently 162 NHS RECs in the UK (as of 25 January 2009). There are two main types of REC, recognised and authorised - the National Research Ethics Service (NRES) and the Integrated Research Application System (IRAS).

In 2007, the National Research Ethics Service (NRES) was launched in the UK. NRES comprises NHS RECs and the former Central Office for Research Ethics Committees (COREC). The NRES has the following remit (see www.nres.npsa.nhs.uk):

- provides ethical guidance and management support to RECs;

- delivers a quality assurance framework for the National Research Ethics Service;

- works with colleagues in the UK to maintain a UK-wide framework;

- works with colleagues in the wider regulatory environment to streamline the processes.

The Integrated Research Application System (IRAS):

- is a single system for applying for permission and approval of health and social care/ community care research in the UK (a standard application form used by all NHS RECs);

- enables a person to enter information about their project once, instead of duplicating information in separate application forms;

- uses filters to ensure that the data collected and collated are appropriate to the type of study, and consequently the permission and approval required;

- helps the applicant to meet regulatory and governance requirements;

- retains familiar aspects of the application system. 
Within the NHS RECs, some committees are flagged as competent to review children's research. The NRES Standard Operating Procedures for RECs do not include much information specifically dedicated to children's research, with the exception of including a requirement to obtain advice by somebody with expertise in paediatric care in the case of CTIMP applications involving minors (2.49ff) (see www.nres.npsa.nhs.uk/news-and-publications/publications/standard-operatingprocedures). However, NRES provides a separate guidance document for researchers and REC members (see www.nres.npsa.nhs.uk/applicants/guidance). Its Section 1.2: 'Children' and Section 3: 'Guidance for Design of Information Sheets for Children/Young People' contain brief general considerations for children's research, highly detailed information on aspects of the informed consent process and supporting documentation for children from different age groups. Appendix $\mathrm{H}$ of the same document provides a summary and extracts of various relevant UK documents on children's research. The focus of this NRES guidance document is health research, in keeping with NRES's link to the NHS. What characterises the information on informed consent in children's research is:

- the importance given to a detailed step-by-step guide through the documentation;

- the distinction between three age bands with exact age boundaries;

- the importance given to ensuring age-appropriate delivery of information by detailed guidance;

- the possibility of exemption from parental consent for minors who fulfil the Gillick competence criteria for non-clinical trials research (however, cautioning that the application of Gillick competence for research participation, as opposed to healthcare treatment, is currently legally untested).

In contrast to the highly regulated NHS review system, the implementation of RECs in the academic sector has been largely left to each individual academic institution; accordingly, a large proportion of children's research is not covered by the strictly regulated NHS system, even though existing guidelines for Social Science Research (e.g. the ESRC Research Ethics Framework, 2006) aim to provide some measure of consistency (see www.esrc.ac.uk/ESRCInfoCentre/Images/ESRC_Re_ Ethics_Frame_tcm6-11291.pdf).

\section{Denmark}

Denmark has a system of Research Ethics Committees (RECs) with 9 regional committees and a national committee (the Danish National Committee on Biomedical Research Ethics). The Danish REC system can be characterised by four main features (see www.cvk.sum.dk/English/ actonabiomedicalresearch.aspx):

- RECs are regional and not institutional;

- there is a central national REC above the regional RECs;

- RECs are relatively small and have a large proportion of lay members;

- approval of multi-centre trials is streamlined.

There is no REC system for research outside the health sector. In 1990, it was concluded that RECs were not necessary in the areas of social sciences or humanities since several kinds of social science are important for policy formation and should therefore not be too constrained by ethical demands. It was also stated that the potential for harming research participants in the social sciences is much lower than in the health sciences.

According to Danish law, all biomedical research projects in Denmark involving humans, or any kind of human tissue, need permission from a regional ethics committee. The investigator of the research project must apply for permission from the regional REC. The application should conform with the Guidelines about notification of a biomedical research project to the committee system on biomedical research ethics. An electronic application form (see www.drvk.dk/anmeldelse) may be sent, in addition to sending a hard copy. The review of the application by the regional REC takes place when a complete and valid application has been submitted. A valid application must include (1) application form; (2) the clinical trial protocol; (3) subject information and the informed consent procedure; and (4) a short résumé in lay terms. Applicants whose project is rejected by the regional REC can appeal the decision to the Danish National Committee on Biomedical Research Ethics. 
The 2003 Act governing the Danish REC system does not have a comprehensive separate section to deal with issues arising in children's research. However, it addresses children's research issues specifically in Paragraph 17, which discusses research with non-competent populations, and in Paragraph 19, which deals with mature minors. In both Paragraphs, the issue of informed consent is the primary concern. The issues addressed include:

- the importance of surrogate consent that is made purely in the interest of a child;

- the importance of involving the minor in the informed consent process through a qualified person who has experience with communicating information in an age-appropriate way; information given needs to include the possibility of withdrawing at any time;

- the importance of research projects with minors being reviewed by somebody with paediatric expertise;

- exemption from parental consent for mature minors over 15 years of age, depending on the nature, risk and harmfulness of the research.

\section{Australia}

There are more than 200 Human Research Ethics Committee (HRECs) in Australia, whose role is the ethical oversight of research involving humans. HRECs all function in accordance with the guidelines outlined in the National Statement on Ethical Conduct in Human Research (2007), issued by the National Health and Medical Research Council (NHMRC). The National Statement sets out the requirements for the composition of a HREC and relevant ethical principles by which research should be designed and conducted. HRECs are usually established by organisations that conduct research involving humans. Universities and hospitals are the most common of these. Before conducting research that involves humans, approval from the specific HREC must be granted. HRECS review proposals for research to ensure that they are ethically acceptable and in accordance with relevant standards and guidelines, monitor the conduct of research and deal with complaints that may arise. The Australian Health Ethics Committee (AHEC) is a principal committee for the NHMRC and provides support to HRECs. The AHEC advises the NHMRC on ethical issues relating to health and develops guidelines for the conduct of research involving humans. AHEC monitors and advises on international developments in health ethics issues through liaison with relevant international organisations and individuals, including the World Health Organization.

The National Statement includes Section 4.2 dedicated to research with children and young people, and Section 4.3 dedicated to research with people in dependent and unequal relationships. The importance of the realisation of children's research is highlighted, especially in the areas of health and education. The specific provisions include (see www.nhmrc.gov.au/publications/ethics/2007_ humans/contents.htm):

- distinction between different levels of vulnerability and maturity;

- importance of a consent process that involves both parents and children, but provision for exemption from parental consent for mature minors under certain circumstances;

- importance of research that is beneficial to children generally;

- possibility of parents giving 'standing consent' for research conducted in schools;

- importance of involving school communities in deliberations about participation in school research;

- the challenge of the effects of power in dependent relationships;

- danger of over-researching a population because it is easy to access;

- danger of negative consequences for research participants due to results or non-cooperation in research.

\section{New Zealand}

The New Zealand Health and Disability Ethics Committees (NZHDECs) issued guidelines called Operational Standard for Ethics Committees in 2006, designed to promote flexibility and consistency in ethical review throughout New Zealand. The Operational Standard derives its public authority from the terms of reference of ethics committees established by the Minister of Health under 
Section 11 of the New Zealand Public Health and Disability Act 2000. Those terms of reference have precedence over the Operational Standard on any point of conflict. The Operational Standard forms the basis for monitoring the operation of ethics committees, which review research and innovative practice, and provide advice on ethical issues relating to clinical decisions about the treatment of specific individuals. All ethics committees reviewing health and disability research in New Zealand should be approved to the requirements of their terms of reference and the Operational Standard by the Director-General of Health or the Health Research Council (HRC) to ensure the attainment of appropriate standards and best practice.

A national application form with minimum requirements regarding written consent and consumer information has been developed (see www.ethicscommittees.health.govt.nz or www.hrc.govt.nz). All proposals must be submitted to ethics committees on this form. Research proposals that are to be carried out in a single ethics committee region should be sent to the administrator responsible for that ethics committee. Research proposals for research that is to be carried out in more than one region or nationally should be sent to the administrator responsible for the multi-region ethics committee.

In relation to clinical trials, separate applications must be submitted for each of Phase I, Phase II, Phase III or Phase IV trials. Each application is subject to review by an ethics committee. Registered health professionals may need to complete one of the following forms:

- Form A: Declaration of Eligibility of a Clinical Trial for Accident Rehabilitation and Insurance Corporation Coverage.

- Form B: Declaration of Provision of Compensation for Injury for Participants in a Research Study for a Pharmaceutical Company.

Appendix 1 of the Operational Standard includes guidelines for health research with children. It acknowledges the importance of conducting research with children, while ensuring their protection and legal consent from the child, parent or guardian. It states that all research involving children needs to be submitted to an ethics committee. The guidelines are based on six principles, taken from the Guidelines of the Royal College of Paediatrics and Child Health 1999 and the European Convention for the Protection of Human Rights and Dignity of the Human Being with Regard to the Application of Biology and Medicine 1996.

\section{Issues for current models}

The international examples of national research ethics governance discussed above demonstrate the range of models that have developed. While in many countries there have been voices in favour of a centralisation of the ethics review system, more thoroughly centralised review systems seem to have been mostly implemented in small countries so far. In most cases, the implementation of general review requirements or of a centralised system of review have only had partial scope, i.e. have been applied to only certain research sectors and organisations (e.g. healthcare providers) or types of research (e.g. clinical trials research, research in institutions that are supported by certain funding bodies). Whether this limited implementation of centralised review is mainly due to merely practical obstacles or whether there are more fundamental reasons remains open. The following discussion will review some of the issues arising in relation to current models of research ethics governance.

\section{Between diversity and standardisation}

Standardisation is not restricted to the case of thoroughly centralised review systems. Any governance approach to research ethics has to address the problem of balancing diversity and locality issues, on the one hand, and the issue of standardisation and quality assurance, on the other. Even in an institution-based review system, the achievement of an effective governance system that ensures an appropriate level of standardisation across RECs is a core concern. 
At the early stages of the development of REC systems, it is usually the responsibility of each individual REC to develop its terms and procedures, and to come to REC-specific agreements on ethical standards of review. At later stages, additional standardising elements might need to be taken into account that have an impact on the functioning of RECs, even if no thorough restructuring takes place and the same committees continue working. In many cases, national funding bodies or some other national entity with responsibility for research develop binding guidelines for ethical review. Additional potential tasks of a governance system that might provide some standardisation include the development of a central administrative body, generally binding standing operating procedures, a unified application form, standards for REC member training, monitoring structures and procedures, and in some cases a centralised appointment process for REC members. Some combination of these elements is frequently mentioned in proposals for reform of research ethics governance systems, as, for example, expressed at the 2007 EC-EMEA Clinical Trials Conference (see www.emea.europa.eu/pdfs/conferenceflyers/clinicaltrials/report.pdf) and the 2005 'Facing the Future Together' Conference on Research Ethics Committees in Europe (see http://ec.europa.eu/research/conferences/2005/recs/pdf/report_en.pdf). Particular emphasis is often given to the implementation of an effective administrative body (Downie, 2006; Fitzgerald and Phillips, 2006).

In some places, standardisation of the review process has led to the implementation of a central body with important responsibilities for governing ethics review in a jurisdiction. Different models of centralisation have been proposed in the debate, including the implementation of national RECs, regional RECs, subject area or research-type specific RECs, or other types of centralised structures (Christian et al, 2002; Downie, 2006; Emanuel et al, 2004; Gandhi, 2005; McWilliams et al, 2006). Alternatively, governance systems might focus primarily on the implementation of a central governing body without necessarily having drastic effects on the number of committees that are in operation (Fitzgerald and Phillips, 2006). However, in general, recommendations for a reduction of ethics committees is quite common in the debate (ACSTI, 2006; Department of Health, 2005); in the Irish context, this was most recently emphasised in the ICRIN report of 2008.

Thorough centralisation has been implemented, for example, in New Zealand and Denmark, where national guidelines govern a structure that includes a very limited number of regional committees which are appointed following central appointment processes. In the UK, all research conducted in the NHS has been under the authority of a single body for several years (previously, the Central Office for Research Ethics Committees (COREC) and now the National Research Ethics Service (NRES), which presently governs a number of additional bodies and envisages these to increase further in number in the future). Frequently, a major driver of reforms of the REC governance system are the limitations of the institution-based system in reviewing multi-centre studies. Arrangements to streamline multi-centre study review frequently include mechanisms of allocating primary review authority to specific committees, or to outline arrangements between committees that will cut down on the overall review burden. In Europe, the Clinical Trials Directive has had an impact on the research ethics governance of multi-centre clinical trials, requiring a single review of such trials with only limited input from the individual trial sites (EU, 2001). However, this Directive has been implemented in different ways in different European countries and may in some cases require assessment by a full local REC (NRES, 2007).

Despite the increasing drive internationally towards standardisation, institutional diversity still plays a significant role. Institution-based RECs that are created and staffed by the institution are still the norm, not the exception, internationally. Frequently, standard operating procedures, application forms and training are developed and organised by RECs themselves, in line with general guidelines, but with some leeway to incorporate elements that do justice to specific legislative situations and other local conditions, often specific to individual institutions. Local conditions for institutions might include the ethos of the institution (frequently considered especially important for faithbased institutions), likely characteristics of researchers and area of research in the institution (e.g. disability or children's research). Even where a non-institution-based REC is responsible for review of research proposals for this institution, some form of 'locality assessment' is frequently 
considered essential as an element of the review process in addition to the original ethical review, to ensure the adequacy of REC review to the particular characteristics and conditions of the institution. This might just include the mere assessment of feasibility of research projects in the institution at a particular point in time, but it might also go further and include the assessment of compatibility with values and ethos of the institution and might even involve an additional committee process (Edwards et al, 2007). The value of diversity and adaptation to individual institutions has also been supported by those who see institutional RECs as one way of representing the institution's values and facilitating institutional buy-in and ownership of the ethics review process. However, if the institutional assessment is not carried out in the context of a governance model that ensures a sufficient degree of standardisation, quality control and streamlining across RECs, there is a significant danger that research might be impeded by procedural obstacles (as especially in the case of multi-centre studies) or not be reviewed appropriately.

\section{Governing different research sectors and research fields}

As already indicated above, in many countries research ethics governance systems do not treat all research equally. While in most countries the area of clinical trials research is governed quite strictly - which in the EU falls under the European Clinical Trials Directive (EU, 2001) and the earlier Oviedo Convention (Council of Europe, 1997) - other forms of research are frequently under less ethical scrutiny and relevant structures may not be put in place for some areas of research. This is especially pertinent to the area of children's research. Despite the existence of numerous documents and initiatives on children's rights - from the UN Convention on the Rights of the Child to the EC Communication 'Toward a European Strategy on the Rights of the Child' (2006) and the expected EU Strategy on Children's Rights, all of which emphasise the right of children to social participation - the specific area of children's research has not attracted much attention. There is a concern that as a consequence not all research participants, including especially children, receive the level of protection that they should be entitled to (Evans, 2004).

One distinction that can be made in this context is the distinction between the health and the academic sectors, which are constituted by institutions that differ in the services that they provide, the professionals that engage in research and the purposes, areas and methods of research. However, there is a significant overlap of both sectors regarding the large field of health research, which frequently causes problems in relation to governance. A significant proportion of health research is conducted with patient populations that are often considered to fall primarily under the jurisdiction of the healthcare institutions, while academic institutions might consider themselves to also have a responsibility for review of such research. Problems with clarifying the remit of RECs in both sectors, especially for research that might involve members from both sectors, are not uncommon and can lead to multiple review by committees in both sectors, especially regarding research outside the clinical trials system where the research ethics review system has been more streamlined than for other types of research.

In systems with a national healthcare system, the healthcare sector may be governed by a single governing body and accordingly it might be somewhat easier to implement uniform research ethics requirements for the entire sector. This is currently the case in the UK regarding all research involving the NHS. In Ireland or Canada, such uniformity has not been created so far. Regional committees, as in New Zealand or Denmark, might be a solution to the potential conflict between RECs in different sectors; however, in these countries the regional RECs only govern health research, i.e. academic non-health research does not currently appear to fall under their remit.

In contrast, standardisation in the governance of academic institutions has been somewhat less pronounced, perhaps due to the particularly strong emphasis on academic freedom and institutional self-governance in this sector. In the UK, there is little standardisation of academic RECs so far, even though an independent Association of Research Ethics Committees (see www.arec.org.uk) is interested in improving communication and development of standards of good practice in this field. 
Where standardisation in this sector has been achieved, it has been due to the influence of research ethics funding bodies, as for example in the USA and Canada where research ethics requirements for institutions are linked to the receipt of funding from certain national research funding bodies. When institutions receive funding from these funding bodies, one of the conditions for funding is that all research, not just the funded research, within that institution undergoes research ethics review that fulfils certain requirements.

Such general review requirements for academic institutions that are linked to the receipt of funding from certain agencies have been criticised, especially because in these cases funding often only applies to certain types of research, but all research within the institution might have to conform to standards that might not have been developed with these types of research in mind. One particular concern in this context has been the adequacy of ethical standards applied to research in the social sciences and humanities, especially regarding a broad range of qualitative methodologies. Submitting such research to research ethics requirements that have been developed following the model of ethical issues encountered in clinical trials has been particularly strongly and widely criticised (Dingwall, 2007; Howitt, 2005; Ramcharan and Cutcliffe, 2001; Richardson and McMullan, 2007; Van den Hoonaard, 2006b). These criticisms have been taken up to a certain extent in some jurisdictions, where special reports have been published on ethical characteristics of such research (ESRC, 2006; PRE, 2004; SSHWC, 2006 and 2007b). The process of inclusion of social science and humanities research seems to have been particularly extensive in Canada, where relevant concerns are now reflected to some extent in recent versions of the Canadian TCP (see http://pre.ethics.gc.ca/eng/policy-politique/initiatives/reports-rapports).

Finally, especially in REC systems that do not comprehensively cover all research, other bodies outside the health and academic sectors might step up to provide research ethics review for research in areas that either do not fall under the remit of other committees or that might be considered as requiring specialised attention. While the more frequent problem is the multiple review of the same proposal, in some cases researchers might have the opposite problem - of not being able to access REC review for research that would require research ethics review (0'Beirne et al, 2007). This can be the case for research by companies that neither involves academics nor members or patients of healthcare institutions, and therefore does not offer easy access to either of these branches of the research ethics infrastructure. In some cases, this problem is solved by using independent or commercial RECs. Alternative ethics review bodies might also be created by professional associations and patient organisations. The implementation of such additional bodies can be motivated by the same problem of being involved in research that falls outside the usual remit, but might also have to do with the self-understanding of such bodies that they have a particular responsibility to the groups they represent. They might perceive the implementation of independent RECs as a possibility to tailor review requirements especially to the needs of their stakeholders.

\section{The REC bureaucracy: Costs and burdens}

One of the most frequently voiced concerns regarding existing research ethics governance systems is related to the danger that on balance the implementation of a research ethics bureaucracy has more costs than benefits. Much of the research ethics literature that has been produced from the perspective of researchers focuses on the extent to which REC review requirements represent an undue burden that impedes rather than facilitates the conduct of valuable research and does not fulfil its stated primary goal of protecting research participants (Burris and Moss, 2006; Fleischman, 2005; Fost and Levine, 2007; Wald, 2004). Researchers argue for the need of looking beyond the mere compliance model towards increased efforts of constructive cooperation with researchers (Connolly and Reid, 2007). Some claim that the current REC system has led to the existence of an inflexible normative discourse that potentially marginalises essential ethical concerns and distracts researchers from paying attention to significant ethical considerations in their research (Cannella and Lincoln, 2007; Haggerty, 2004; Halse and Honey, 2007). 
Researchers have pointed out the costs involved in REC review in terms of a number of variables:

- time spent in preparing research ethics application;

- time spent waiting for feedback and approval;

- number of required clarifications, amendments and resubmissions;

- costs in terms of researcher time;

- costs in terms of application materials and fees.

Costs have been identified as particularly high for multi-centre studies, especially in those systems where several RECs review the same proposals and where researchers are required to meet a range of different and frequently inconsistent demands for amendments and different timelines (Burman et al, 2001; Emanuel et al, 2004; Estlin and Ablett, 2001; Larson et al, 2004; McWilliams et al, 2003; Roberts et al, 2004; Seiler et al, 2007). One of the most frequently highlighted issues, and one that has been addressed by many governance systems in recent years, is the need for a more streamlined process of review of such studies, both nationally and - more problematic internationally (Christian et al, 2002; Hearnshaw, 2004; Hirtle et al, 2000; Pentz and Khayat, 2004; Pinkerton et al, 2002).

This need for a more systematic organisation of the REC system is acknowledged by many guidelines. For example, the WHO (2000, p. 2) Operational Guidelines state, 'Procedures need to be established for relating various levels of review in order to ensure consistency and facilitate cooperation. Mechanisms for cooperation and communication need to be developed between national committees and institutional and local committees. These mechanisms should ensure clear and efficient communication'. In some cases, this is primarily envisaged to be realised through better communication structures and information flow between committees; in other cases, a more thoroughly streamlined system is seen as a solution. However, even where national guidelines mandate the acceptance of review decisions from one committee by other committees, there is evidence that this does not always translate into reality (Roberts et al, 2004).

One frequently mentioned concern is the variability and unpredictability of REC review. Researchers frequently report cases of capricious decision-making by RECs. Some of the problems that researchers encounter in this context might be due to their unfamiliarity with the review processes and the expectations and narratives that guide REC members' decision-making processes (Fitzgerald and Phillips, 2006). However, there has also been a considerable amount of research conducted into different parameters of ethics review by different RECs that proves that the impression of unpredictability is real. Probably the most frequently replicated finding has been of considerable inconsistency regarding a broad range of parameters (Edwards et al, 2007; McWilliams et al, 2003). The discrepancies have been particularly pronounced in research ethics governance systems where few binding regulations were in place. However, even in systems where efforts had been made to streamline review and ensure greater consistency between different committees, significant differences still remain (Evans, 2004). While the issue of multiple applications and timelines can be addressed relatively easily through reforming the review process, the possibility of improving agreement on issues of ethical decision-making itself is more doubtful. Some propose improved training and quality assurance through peer review as a potential solution to preventing such diversity (Recommendation 8 in Department of Health, 2005). However, as some authors have pointed out, ethical decision-making is characterised by an irreducible component of individual moral judgement that might be reduced to a certain extent by ethical guidelines, but will never be fully eliminated. The joint deliberation process within the committee context might magnify this effect, while at the same time constituting an essential feature of sound ethical decision-making (Edwards et al, 2007).

Significant efforts have been made internationally in recent years to improve and streamline the REC process. Some systems have undergone prolonged review and revision processes. These attempts have mostly focused on the issue of standardisation of review processes. They frequently include: 
- creation of a central body with responsibility for research ethics governance (however, with widely varying status, remit and authority);

- development of binding standard operating procedures and ethical review guidelines;

- standardisation of application forms;

- improved accessibility of relevant documentation for RECS and researchers through online facilities.

Many of these modifications to the review system still need to be evaluated. While some burdens of the review process are likely to be improved by these measures, others are likely to remain. It has been pointed out that some of the concerns in relation to the burdens of REC review might be inevitable side-effects of implementing a bureaucracy with primary focus on the establishment of a hierarchical structure with oversight powers (Bosk, 2007; Dixon-Woods et al, 2007). Accordingly, efforts to improve the effectiveness of such a bureaucratic structure are not likely to eliminate its inherent problems (Zywicki, 2007).

The Irish REC system is currently characterised by the predominance of local RECs that mostly represent individual institutions, such as hospitals or universities. These RECs have mostly been developed from the ground up, i.e. in response to a perceived need of each individual institution to implement REC review structures within the institution, rather than as a consequence of a top-down process demanding such implementation. With the exception of the Clinical Trials Committees, there is currently no governance structure in place that regulates the work of RECs in Ireland.

\section{Main concerns}

- Wide range of governance models in existence internationally, but no international consensus on best practice for REC governance systems.

- Tendency towards increased standardisation and centralisation, even within institutionbased systems.

- Limited scope of most governance systems, frequently excluding academic sector and non-health research, especially social science research.

- Inconsistency across RECs and resulting obstacles, especially in review of multi-centre studies, as important driving force for governance reforms. 


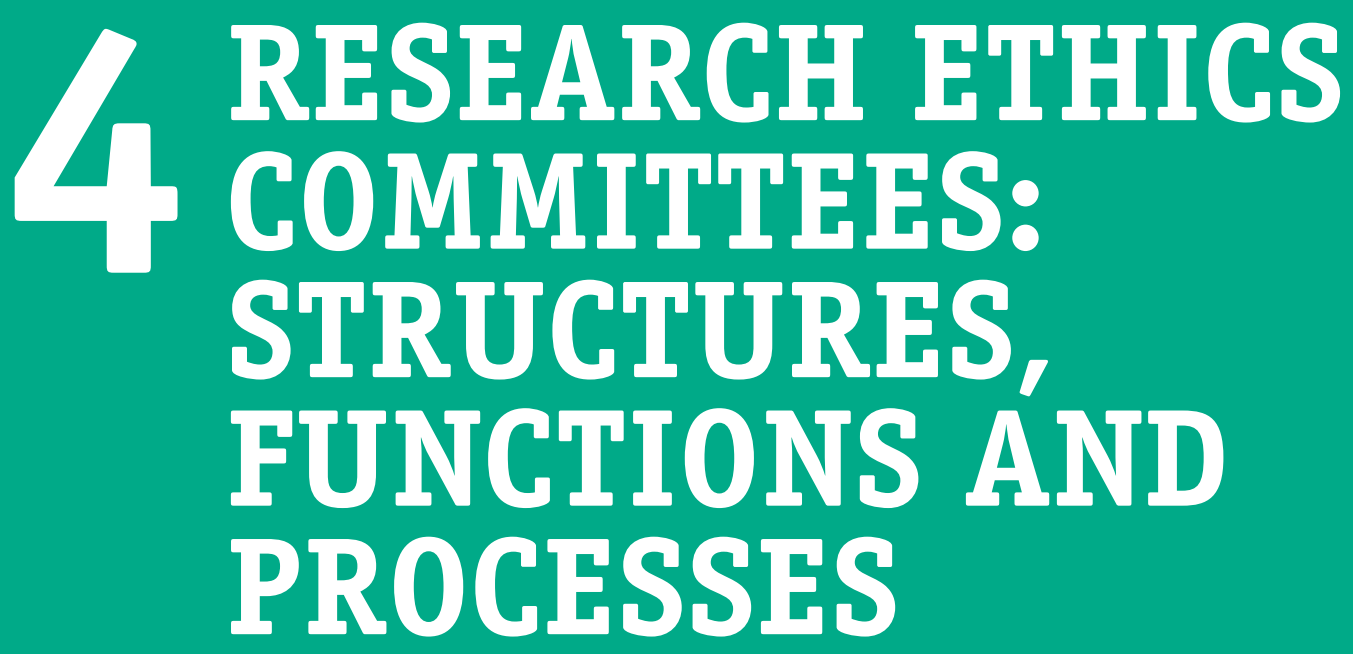




\section{Irish Research Ethics Committees - The landscape}

Research Ethics Committees (RECS) have been a much less prominent part of the research landscape in Ireland than in other countries, especially in English-speaking countries. While a number of hospitals have long had ethics committees in place to review medical research and clinical trials, the growth and development of RECs was particularly pronounced during the last decade and received broader attention when the Irish Council for Bioethics published its Operational Guidelines for Research Ethics Committees: Guidance 2004 (ICB, 2004). During that time, the Irish Council for Bioethics created a list of RECs in Ireland, which it updates when committees report relevant changes (see www.bioethics.ie/index.php/research-ethics-committees-ireland). However, as the Irish Council for Bioethics is not an official governing body of Irish RECs, the accuracy of this resource depends on self-reporting by committees. In several sectors, especially the academic sector, significant changes have been taking place over the last few years that have not yet been captured by (or reported to) the Irish Council for Bioethics.

Generally, RECs in Ireland can be classified into several different categories:

\section{- Health and social care committees}

HSE committees, either hospital-based or regional;

Committees in the voluntary and private sector;

Clinical Trials committees.

\section{- Academic committees}

Central committees for academic organisations;

Committees for sub-units within larger academic bodies.

\section{- Other types of committees}

Committees linked to relevant professional associations;

Committees linked to other bodies.

\section{Healthcare RECs and Clinical Trials RECs}

Among the healthcare-related committees, hospital-based RECs are mostly based in Dublin, whereas outside Dublin they take on, either officially or unofficially, the function of regional committees. Based on the list of RECs compiled as part of this study (see Appendix 2), there are 29 HSE committees, 18 of which are located in Dublin and 11 in the different regions of the HSE. (An anomaly is one of the RECs that reviews proposals relating to HSE institutions in the region, but is subsumed under the governance structure of one of the academic institutions.) There are also 11 health and social care RECs run by voluntary organisations and 6 RECS run by other bodies.

Among these committees, one important further distinction is between those RECs that have been approved to review clinical trials according to the European Clinical Trials Directive and those that do not have this authority. The majority of the Clinical Trials RECs are also HSE RECs. Currently, there are 13 RECs that are entitled to review clinical trials, which involve studies that use medical substances and interventions with either clinical populations or healthy subjects. These RECs are part of a governance structure that exists separately from the other RECs and is based on the requirements stated by the European Clinical Trials Directive. These committees need to fulfil certain requirements regarding the composition of the committee, quorum, turnaround times and documentation to be reviewed. In the case of these Clinical Trials RECs, applicants can submit their application to any of the eligible committees and the approval they receive will, in principle, be valid for any institution in which they intend to realise their study. This structure was designed to reduce the organisational burden in the realisation of clinical multi-centre studies, a characteristic format of clinical trials. The Department of Health and Children is currently the responsible body for the Clinical Trials RECs and receives their annual reports. However, their remit does not include the central distribution of applications, so that it remains up to the applicants to choose the committee that they will apply to. However, in most cases these applications will have 
to go through a further process of assessing the suitability of the research in each of the chosen individual trial sites. Commonly, this does not require full REC approval on these sites; instead, the documentation would be submitted to management or some other channel for approval.

\section{Academic RECs}

Among the academic RECs, the situation is extremely varied. Currently, there is no explicit requirement on academic institutions to create RECs to review the research of their staff and students. Currently, there are 27 academic RECs on record (RECs on different sites of affiliated academic institutions with distinct academic profiles and programmes are counted separately). The majority of the larger and particularly research-active institutions of higher education are among them, but a number of organisations remain without a REC.

The following descriptions draw on information gathered in the present study through the qualitative data collected from participants who are stakeholders in academic RECs. The models of governance used in these academic institutions vary significantly.

\section{Single central REC}

The majority of academic RECs are central committees, especially in those institutions with smaller student and staff numbers. Among those RECs that contributed to this review, 5 of the committees follow this model. One of the challenges that institutions encounter that follow this model is the increasing number of research proposals coming through the committee. In particular, they face the question of whether student research should be reviewed at all and if so, according to which criteria research should be selected for review.

\section{Hierarchical models}

One alternative approach to research ethics governance is the implementation of a hierarchical review structure, where a single higher level REC works in conjunction with REC sub-committees that are located at a lower level of the institutional hierarchy. These might be faculties, schools, departments or groupings that are not strictly aligned to academic units which review certain types of research. While only 3 of the interviewed committees followed this model at the time of the review, these had mostly been adopted comparatively recently and there were indications that further movement towards hierarchical models may be underway in other institutions that are currently still operating under a centralised system.

A. With ethical review at all levels: According to this model, all RECs conduct review of research proposals. While the sub-committees focus either on student research or on low-risk research, the central committee focuses on staff research, higher risk research or research with particular populations. This model has been implemented in one of the institutions contacted.

B. With ethical review only at sub-committee levels and exclusively policy and governance functions at higher level: According to this model, only the RECs at the lower level(s) are engaged in review, whereas the higher level committee performs research ethics policy functions, based on reporting from lower level committees. This model is realised in 2 institutions, with some significant differences in the degree of integration of the different levels and the extent of research ethics policy development between these institutions. Interestingly, both of these institutions have included representation of animal research committees at the highest level, whereas in the majority of other committees this area of research seems to be dealt with through separate structures.

\section{Independent sub-committee model}

According to this model, the development of REC structures and ethical review is completely devolved to sub-units of the institution that develop their own ethical guidelines, form their own RECs and are fully in charge of their research ethics policies. This model has been realised in one of the academic institutions interviewed. 
A challenge that most of these committees and sub-committees face is the creation of good working relationships between the committee and the diverse academic units or staff members whose research falls under their remit. There are different approaches of outreach, from including representative members of different sub-groups, units in the committee to establishing links with representatives of these units whose task it is to raise awareness of the REC within their units. Those universities that have developed a hierarchical model tend to arrange for formal reporting relationships and representation of those sub-committees that work at the next lower organisational level.

\section{Other committees}

Finally, the remaining grouping of RECs is quite diverse (see Appendix 2). It currently includes 6 RECs, but there are likely to be additional committees that either have not been identified yet or that have come into existence more recently. This group includes professional bodies in different medical specialties that focus on reviewing research by their members and trainees. It also includes Government-related bodies that focus on reviewing research that involves research with vulnerable populations in their care or recipients of their services. In addition, it includes a funding body.

\section{Characteristics and working conditions of RECs}

Currently in Ireland, 72 RECs have been documented; some academic institutions were hosting several RECs (see Appendix 2). During the research process for the present study, information was received from 46 of these, either through response to the questionnaire or by telephone contact with the chairperson or administrator. The majority of these RECs are based in health and social care organisations and are located in Dublin. Of these 46 committees, 2 are exclusively concerned with research ethics governance in their institutions, 1 was at the time of contact still predominantly a clinical ethics committee, and the remaining 43 committees are either currently reviewing research proposals or are in the process of setting up procedures for conducting such review. Among the RECs represented by the survey respondents were many committees that had been in operation for over a decade. Others had only been set up during the last 5 years; this was especially the case for RECs in academic and voluntary organisations.

\section{Composition of international RECs}

Membership of a REC is usually characterised by a concern for the inclusion both of a range of relevant expertise and of sufficient diversity. The WHO $(2000$, p. 2) Operational Guidelines recommend that States should strive to establish RECs at 'the national, institutional and local levels that are independent, multi-disciplinary, multi-sectorial and pluralistic in nature'.

One of the core concerns in deciding on the composition of a REC is its representativeness for the organisation and the inclusion of the kinds of expertise that will be needed in order to assess the ethical quality of the proposals that come before it. Existing guidelines on REC membership sometimes stipulate a minimum number of members, frequently somewhere between 5 and 8 (CIHR et al, 1998/2005; HHS, 2005; Government of Denmark, 2003; ICB, 2004; NHMRC, 2007) in order to allow for a sufficient diversity of viewpoints in REC deliberations. According to the WHO (2000, p. 2) Operational Guidelines, RECs 'should be multi-disciplinary and multi-sectorial in composition, including relevant scientific expertise, balanced age and gender distribution, and lay persons representing the interests and the concerns of the community'.

Guidelines sometimes include more specific stipulations regarding the type of expertise that should be represented in the membership of RECs (CIHR et al, 1998/2005; HHS, 2005; ICB, 2004; NHMRC, 2007). Common suggestions regarding the composition of RECs concern the inclusion of:

- 'lay' or community members;

- legal members;

- members with ethical training; 
- members with an understanding of those research areas and methodologies that are likely to be reviewed by the committee;

- members whose experience qualifies them to act as subject advocates, especially for vulnerable populations.

Guidelines on REC composition frequently stipulate that a substantial proportion - frequently one-third, but in some cases over $50 \%$ - of members should be lay members (Department of Health and Children, 2004; Holm, 1992; HRC, 1996; ICB, 2004; NHMRC, 2007; WH0, 2000). However, the OHRP's guidelines in the USA and the Tri-Council Policy (TCP) in Canada require only a single lay representative on a committee, albeit with the general acknowledgement that if committee membership increases, community membership should also increase (TCP, B2.1.3). Yet the definition of what constitutes lay member status varies significantly. According to the most restrictive definition, favoured, for example, by the ICB (2004), lay persons would be defined as community representatives who are:

- not affiliated with the institution;

- not from a professional background that is prominently represented in the institution itself;

- not fulfilling another relevant specialist function on the committee (e.g. legal or ethical).

Generally, the practice in many jurisdictions seems to be that genuine community membership is quite limited. Even where substantial lay membership is legally mandated, definitions of what constitutes 'lay' status can differ significantly. De facto, lay members are frequently either members of the institution or belong to a professional group that also fulfils some other specialised function (e.g. legal, ethical or non-medical healthcare professional) on the committee. For example, according to the Irish Regulations in relation to the European Clinical Trials Directive (EU, 2001), lay membership - as defined in Schedule 2 - is compatible both with membership of the institution where the REC is based and the fulfilment of any specialist REC function that does not include clinical research or clinical practice (Department of Health and Children, 2004).

In addition to the question of definition, the experience of lay members and the creation of conditions for successful integration in the REC deliberations have been the subject of considerable concern (Anderson, 2006; Dyer, 2004; Glasby and Beresford, 2007; PRE, 2007; Rivera et al, 2004; Roseman, 1987; Sengupta and Lo, 2003; Slaven, 2007). Other concerns regarding the composition of RECs include the question of the representation of minorities or women on committees (Campbell et al, 2003; CIHR et al, 1998/2005; Dickenson, 2006; Moerman et al, 2007; Schuppli and Fraser, 2007), the representation of advocates for vulnerable groups (OSTP, 1991) and generally the question of representation of sufficient methodological or subject area expertise.

The required scientific expertise may differ depending on the institution within which the REC is located and the purpose of the committee. For RECs located in healthcare institutions, there is usually the requirement that a range of healthcare professions should be represented (HHS, 2005), including, for example, medical doctors from a range of specialty areas, nurses, allied health professionals or psychologists. For RECs located in academic institutions, there is frequently an explicit or implicit requirement that they need to represent the different academic units or research areas that the committee covers.

Contentious topics in the debate have included the question of how RECs could best address the different ethical issues that arise in different areas and methodologies of research, e.g. medical and natural science research, on the one hand, and social science research, on the other, with particular attention to qualitative research methodologies (Azar, 2002; Connolly and Reid, 2007; Ells and Gutfreund, 2006; Hemmings, 2006; Hesse-Biber and Leavy, 2006; Jacobson et al, 2007; Lincoln and Tierney, 2004; Nelson, 2004; Ramcharan and Cutcliffe, 2001; Stevenson and Beech, 1998; Tolich and Fitzgerald, 2006). An additional challenge that faces RECs in relation to ensuring review expertise and adequacy is the challenge of combining competence-building and continuity of membership with the avoidance of the establishment of unquestioned and potentially biased review routine. The limitation of terms of office, as frequently mandated by guidelines, is generally 
considered the best antidote to this problem, by allowing a regular influx of new members who can bring fresh perspectives to the table. However, this also creates the need to recruit and train adequate replacements, which can be a challenge especially in smaller organisations where the number of potentially suitable members can be limited.

\section{Composition of Irish RECs}

According to the survey data from the present study, membership of Irish RECs ranges from 4 to 21 members, with a mean of 13.0 (s.d. $=4.2$ ). Within these committees, $91 \%$ reported requiring a quorum of approximately half of the membership (between $25 \%-77 \%$ ) before a decision on a review could be made.

Over $70 \%$ of RECs had a membership with the following professional backgrounds:

Legal advisor

Medical doctor

Nurse

Lay person

Less than one-third of RECs had a membership with the following professional backgrounds:
Psychologist
Ethicist
Statistician

Religious representatives were included in 38\% of the RECs surveyed. Single RECs reported membership of post-graduate students, administrators and patient representatives. Multiple membership from the same professional background on a REC was most common for medical members, scientists, allied health professionals and lay persons. The high average number of lay members was partly due to their mandated one-third presence on the Clinical Trials RECs. The data indicate that the surveyed RECs fulfil the most common international requirements for membership of committees in terms of numbers and membership mix; the surveyed Clinical Trials RECs fulfilled the membership requirements of the EU Directive.

This survey item was designed to capture professional backgrounds and did not allow for multiple inclusion, and therefore did not identify the number of children's researchers on participating RECs. From the interview data, it was evident that all RECs with a large proportion of children's research had one or several experts on children's research on the committee; other RECs generally did not identify membership from this category as particularly relevant for their work.

\section{Stakeholders' perspectives on Irish RECs}

It was agreed by participants in the qualitative research of the present study that the most important criteria for REC membership was that they had at least an interest, if not experience, in doing research themselves:

'... people who are interested in doing research themselves and quite a lot of our Committee members are people who also do research.' (A)

Interest in research ethics by REC members was also valued:

'It sounds obvious, but you do need somebody that is actually interested in research ethics.' (AC)

It was noted that RECs should be composed of a variety of professional or academic disciplines. One Chair of an academic REC (AC) said committees needed a 'spread of expertise', while another in the same position commented:

'It's very important that you have a wide variety of experts on the panel.' (AC) 
The need for a range of expertise was reinforced by a children's researcher (CR), who explained the reason for this from their perspective:

'Because the nature of research ethics committees is the medical sciences ... I would feel that if you don't have people with a background in social research around that table, you get asked very scientific-type questions ... social scientists have a different perspective in a lot of cases.' (CR)

\section{Specific REC membership}

A number of specific disciplines or areas of expertise were identified as important to be represented or available to RECs. One of these was medicine:

'We have always felt we should have somebody who is coming from a medical background and essentially somebody who might have an interest in medical ethics or perhaps bioethics.' (AC)

This also highlights the place of ethics expertise, which was generally considered as an area of interest for specified disciplines. Interestingly, very limited reference was made to the role of ethicists per se, although one hospital Chair ( $\mathrm{HC}$ ) acknowledged, and appears to have valued, the participation of an ethicist on the committee:

'Within our committee, we also have a professional ethicist and we feel that this is very important in ... professionalising what we do.' (HC)

It was felt to be important to have legal representation on RECs, either a lawyer or member of the Garda Síochána or failing that, access to an external source of legal advice such as 'college lawyers'. As well as citing areas of expertise that should be included and available to RECs, one Chair identified an area of exclusion:

'We have stayed away from anybody religious. There is nobody religious on the committee and this is the way I want to keep it.' (HC)

Committees and researchers were in agreement on the importance of having external or lay members on the REC. However, these had sometimes proven difficult to recruit:

'We do not have any lay members. This has been our difficulty. We've struggled with the concept of having lay people on our ethics committee and where do we get those lay people from; and we've tried.' (AC)

Academic RECs utilised students as lay members. Hospital committees recruited lay members who were either hospital patients (by means of consultants or a patient forum) or hospital administrative staff.

\section{Remit, workload and functions of international RECs}

Most frequently, RECs are organised by an institution that has a stake in the research proposals that will be reviewed, for example:

- research performed by staff members;

- research being performed on members of the organisation, be they patients, clients, staff or students;

- research otherwise related to that institution, e.g. researchers funded by the organisation;

- research on citizens, in the case of regional or national government organised RECs.

However, in some places, independent RECs without any such links are in operation. In contrast to most other types of RECs, such committees can be commercial entities operating for profit. The existence of such for-profit committees has caused some controversy (Coleman, 1982; Emanuel et al, 2006; Shamoo and Woeckner, 2006; Silberner, 1998; Tandy, 1996). While some of the running costs of some committees may be covered by application fees (Department of Health and Children, 2007), most frequently the organisation carries a significant part of the costs, which can be 
substantial (Speckman et al, 2007). Yet usually members perform their service on the REC as a voluntary and unpaid service to the organisation, even though in some cases expenses may be covered or an honorarium might be paid for their services. Sometimes compensation is restricted to external members who are not members of the organisation that hosts the REC. The question of adequate compensation for the REC work has been raised as an important issue, for example, in recommendations by the UK Department of Health (2005). Another concern in this context is the protection of REC members from legal liability (Resnik, 2004).

Especially in RECs with higher workloads, the provision of review services on a voluntary basis can become a challenge. Frequently, committees meet at least on a monthly basis and members might be required to review a large number of applications, which can amount to significant demands on members. Generally, there has been a tendency towards an increasing workload for RECs in recent years (Burman et al, 2001; Gunsalus et al, 2007). Given that according to some criteria, a committee might be considered to have a comparatively low review load if it reviews less than 80 proposals per year (in the UK, according to NRES, 2007, see www.nres.npsa.nhs.uk/ news-and-publications/publications/corporate-publications) or 125 proposals per year (in the USA, according to Office of Inspector General, 1998), the amount of work required even of members of 'low volume' RECs can be quite substantial. One significant factor that determines review load is the definition of which kinds of research require ethics approval. Criticism has been levelled at the expanding remit of ethics review especially over the last decade and the perception that a problematic 'IRB mission creep' has occurred is quite common (Gunsalus et al, 2007; Haggerty, 2004; Nelson, 2004).

In order to keep review loads manageable despite these recent increases, some types of research may be considered not to fall under standard review requirements. Increasing review loads have led to the expansion of expedited review procedures that bypass the full REC review. Expedited procedures based on screening of proposals by a limited number of experts have been recently implemented (e.g. in the UK and within the EU FP7 Framework Programme). However, critics point out that the widespread use of such procedures might undermine the integrity of the ethics review process (Hunter, 2007b).

Student research is another category that is sometimes conceptualised as outside the remit of REC review due to being primarily focused on training and learning rather than on research proper (Central Office for Research Ethics Committees, 2004; NZHDEC, 2006). In some cases, it is considered as being either fully the task of the research supervisor or of review structures other than standard RECs. Review bodies that have been envisaged for student research include, for example, specific inner-departmental review processes for undergraduate student projects (CIHR et al, 1998/2005) or dedicated Student Project Research Committees (SPECs) (Central Office for Research Ethics Committees, 2004). However, this interpretation is contentious and in many places all or the majority of student research has to undergo the same review process as other types of research (Howitt, 2005).

Frequently, exemptions or alternative fast-track review pathways are in place for certain low-risk research protocols with non-vulnerable populations, the parameters of which are discussed in some detail by relevant REC guidelines (CIHR et al, 1998/2005; HHS, 2005; ICB, 2004; NHMRC, 2007; WHO, 2000). The distinction between research, on the one hand, and audit, quality improvement or evaluation, on the other, together with the question of the inclusion or exclusion of the latter in the ethics review process, has been another issue that has received significant attention (Candib, 2007; Casarett et al, 2000; Choo, 1998; Cretin et al, 2000; Doyal, 1997 and 2004; Grady, 2007; Hauswald, 2002; Lynn, 2004; Mold and Peterson, 2005; NEAC, 2003; NHMRC, 2003b; Perneger, 2004; Prideaux and Rogers, 2006; Wilson et al, 1999). Similar questions have been raised regarding research into medical education (Brown et al, 2007).

One additional concern regarding the work of RECs has been the exact definition of their function. While the review of research proposals is usually seen as their main function, many RECs define 
their roles as not limited to the once-off review of individual proposals. Ongoing monitoring or auditing of research projects is one of the functions that has been increasing in significance in recent years and is mandated by most guidelines, at the very least for clinical trials and other higher risk research. In addition, frequently considered functions include the education of researchers and more generally the establishment of a comprehensive research ethics culture in the institution. These are considered to be important to counteract the possibility of fostering a culture of mere compliance among researchers (Taylor, 2007b) and creating potentially adversarial relationships between the REC and the research community (Chalmers and Pettit, 1998; Connolly and Reid, 2007).

\section{Remit and workload of Irish RECs}

According to survey data from the present study, RECs in Ireland met between 2-11 times in 2006 ( 5 committees met only twice, while 6 met 11 times), with a mean of 6.3 meetings during the year (s.d. = 3.2). Monthly or bimonthly meetings of about 2 hours in length were the most common. The majority of committees (72\%) reviewed up to 50 submissions per year, with the overall median being 36; two committees in particular reported a very high workload, of 196 and 278 reviews respectively. Clinical trials committees met more frequently than other committees and had, on average, a higher workload than other RECs (a median of 71 submissions per year), even though in most cases only a fraction of their reviewed proposals were applications under the EU Directive. Hospital and healthcare committees reviewed a median of 42 submissions per year. Academic committees had the lowest workload relative to other RECs, reviewing a median of 36 submissions per year. From the interview with academic sub-committee chairs, it became apparent that many of these sub-committees, whose remit generally includes the review of all student proposals in their area, have a quite high review load when compared with other Irish committees, reportedly between 80 and 200 proposals per year. They commented that in those cases, the high review load contrasts starkly with the near total lack of resources provided for committee functions.

It was reported that up to $80 \%$ of submissions to any REC included children as research participants, with an overall median of $10 \%$. The proportion of studies including children as participants was generally higher in the academic committees ( $1 \%$ median in HSE/healthcare committees, $12 \%$ median in academic committees and $3 \%$ median in clinical trials committees).

Student research is a significant factor in the work not just of academic committees but also of health and social care committees, and comprised up to $90 \%$ of the workload in some committees. Based on survey data, a median of $40 \%$ of the submitted applications to all RECs were for student research; academic committees had a marginally higher rate $(45 \%)$ of student research than did other committees. Most committees (71\%) review submissions for research to be conducted outside the organisation, with the vast majority $(87 \%)$ from researchers based outside the organisation.

\section{Stakeholders' perspectives on remit and workload of Irish RECs}

From the qualitative data of the present study, in relation to workload, it was reported that academic-based RECs generally had quite heavy workloads. Some committees were faculty- or discipline-based; some acted for the institution as a whole; some covered undergraduate and post-graduate research projects; and others were confined to reviewing staff or commissioned/ funded research.

Some RECs appear to have issues identifying and maintaining the boundaries in relation to their ethical remit and the assessment of research proposals. For example, it is reported that some members query the scientific rigour of certain proposals and consider this an ethical problem, while for others this is a research methodology and quality issue, not an ethical one:

'Sometimes there's an argument or discussion among our ethics committee about really where ethics starts and where science starts, and I think ... historically people have kind of looked upon it [ethics] as being quite separate to the basic science. We would take 
a different view on that within our committee, in the sense that ... if we felt that the science behind the project was not acceptable, then we would see that as being part of our ethics remit. But that can cause some difficulty. Some applicants feel that it is not the role of the ethics committee to comment on the science. But I would have to say that we would see it is [its] remit [in] covering ethics to include also the science part of the project. That's actually very important.' (HC)

Hospital-based RECs, or rather institutions as a whole, expressed tension between ethical research and audit:

'For the moment, the most frustrating bit are the overlaps ... what should we deal with and what shouldn't we deal with? On "what shouldn't we deal with", there is an awful lot of paperwork and small projects coming through that really would come under quality assurance or audit headings ... The audit is mandatory for all of us, it is not an option, like research, to a degree. It is a standard of patient care. So, finally, I imagine this is true across the board, it is better for everybody's satisfaction. That kind of stuff, they shouldn't bother the ethics committee with.' (HC)

Similarly, queries are raised in hospital-based committees on the appropriateness of their ethically reviewing community-based research:

'The more frustrating ones are dealing with areas that actually properly do not come under the remit of our committee at all. And this is a massive national issue, in my view ... They [community-based research projects] are not involved in the hospital, they are not involved in hospital staff or patients, they are not even in the hospital catchment area. But the reason they come to us is that they are desperate to do the right thing and they send it to an ethics committee to have the project scrutinised. Where else should they send it to?' (HC)

Committees in academic institutions differ as to whether they require undergraduate and postgraduate projects to be assessed by the REC or merely by an academic supervisor. Chairs of academic RECs, in particular, were keen to emphasise the importance of the REC's role in increasing awareness of ethics among researchers. This awareness-raising remit was perceived to be at individual and institutional levels, and took place incidentally as a result of the existence of the ethical review process.

'I think the very existence of the committee has increased awareness. That is one of the issues that I felt needed to be addressed throughout the college. It has increased awareness of the kind of ethical issues that are likely to arise and increased awareness maybe of the long-term consequences and implications of the actions of researchers.' (AC)

One newer member of a REC noted that it increased their own awareness of ethics:

'Things that I might have said in the past in lectures that are perfunctory as far as ethics are concerned, I am [now] learning how serious an issue it is and the responsibility that an ethics committee has and that it is not some kind of rubber-stamping exercise.' (CM)

An educational remit for RECs was identified by some participants, beyond that of awarenessraising, while others identified the role of RECs in the promotion of research itself:

'... and part of it is about fostering research as well in this country and helping researchers to get through that hurdle. You need some kind of support system and you need people who are professionals.' (A)

The same remit applied in clinical settings, as expressed by the Chair of a hospital-based REC: 'The ethics committee was instrumental in getting our hospital to adopt research as one of its key priority in terms of our best hospital strategy because that actually hasn't been the case before. So I think having research as an integral part of the hospital is very important and I believe the role of the ethics committee is to ensure that this takes place.' (HC) 
The result of tensions in relation to an apparent lack of clarity in the remit of RECs was expressed by a researcher with reference to audit:

"[0ne needs] very clear guidance as to what nowadays is "research" as distinct from "audit", what needs to go to a research ethics committee and what is perfectly OK not to. To some of us who are doing it all the time, it might seem very clear-cut, but actually it is not. And so you end up falling on both sides of the fence and you end up putting things through a research ethics pathway that really don't need to go there at all. And, on the other hand, you end up being half the way down on a research project and you realise "Hmmm, that really should have gone there ..." ' (CR)

\section{Organisation and resources of Research Ethics Committees}

In addition to the membership of the committee that is engaged with the ethical review of proposals, the day-to-day operation of the REC requires administrative support and a certain level of resources for other aspects of its running, which is explicitly acknowledged by most research ethics guidelines (CIHR et al, 1998/2005; HHS, 2005; NHMRC, 2007; WH0, 2000). As the TriCouncil Policy (TCP) states: 'Institutions must ensure that REBs [Research Ethics Boards] have the appropriate financial and administrative independence to fulfil their primary duties' (TCP, B.1.2).

Without such support, the fulfilment of REC responsibilities cannot be guaranteed; accordingly, the widespread lack of adequate support for RECs, despite the guidance of research ethics guidelines, has been criticised and potential solutions have been explored (Derse, 1997). In particular, smaller committees that work on their own within an institution-based system receive only clerical support from the institution. In more strictly governed research ethics systems, or in those committees with a higher workload and those in charge of review of sensitive research proposals, more extensive resources may be allocated for the administration of the committee. Such committees frequently employ one or more full-time administrators and members of staff frequently have higher levels of qualification and specialist expertise in research ethics.

Funding of committees via review fees is one possibility. However, substantial review fees are usually only feasible for well-funded research projects and the level of funding does not always correspond to the ethical characteristics of the project. Some guidelines advise against setting review fees that could impede access to ethics review (NHMRC, 2007). Common practice is that only commercial research or clinical trials are subject to substantial review fees, while individual investigators can frequently access the REC for nominal or no fees. However, it has been remarked that non-commercial clinical trials might be impeded in some cases by existing practices (Sheard et al, 2006). Committees that review clinical trials are usually at least partly funded by the fees they receive for review of clinical trial proposals.

The work of REC administrators can range from clerical work, in relation to the processing and distribution of applications and correspondence, to an administrative role, which may include a broad range of independent and specialist responsibilities. Administrators are usually in charge of record-keeping, database management and monitoring of research, where this is part of the activities of the committee. While in many systems there is significant variability and lack of clarity about the exact scope of REC administrators' tasks, their responsibilities are described in considerable detail in some guidelines, as in $2.73 \mathrm{ff}$ of the UK's Standard Operating Procedures (NRES, 2007). In cases where a standardised national electronic application system is available nationally, some of these tasks are facilitated by such systems. Specialist commercial software for application management might be available (as in the USA through ProIRB, see www.proirb.com). However, in many jurisdictions it is still up to individual institutions to develop their own recordkeeping and monitoring approach and required database software.

As administrators are often the first contact point for applicants, they are also frequently involved in advising applicants on the preparation of their proposals and the ethical concerns that they need to take into account in their research projects. Some administrators take an even broader 
strategic role, such as developing research ethics policies, engaging in liaison work with other units within the organisation and organising training events. Such administrators could be expected to have received more substantial training in research ethics to fulfil these roles, as, for example, provided by the NRES in the UK or through the certification programme for Certified IRB Professionals (CIPs) in the USA.

It has been highlighted that the work of RECs themselves needs to be monitored to ensure the quality of their work. While some are convinced that such monitoring functions should be the responsibility of an independent auditor (e.g. a central REC governance body), others highlight the importance of self-monitoring of institutions (NHMRC, 2007). In this context, it has been pointed out that care should be taken to develop appropriate criteria that accurately measure the quality of ethics review (Burris and Moss, 2006; Emanuel et al, 2004; Fleischman, 2005) and that might need to include criteria for research ethics quality over and above mere compliance standards (Taylor, 2007b).

\section{Organisation and resources of Irish RECs}

According to the survey data, resources represent a major challenge for the work of RECs in Ireland. While most committees have some access to administrative support, in many cases this is minimal. The median administrative time per week is 2 hours, although $20 \%$ of committees do have a dedicated administrative support in excess of 15 hours per week - and in two cases, up to 60 hours per week. In the majority of cases, administrators are not paid through a dedicated REC budget. Fewer than $10 \%$ of committees even have a dedicated budget; rather, the REC work is included in a range of other duties and frequently competes with these duties. Committees that review clinical trials have considerably more administrative support than other RECs. All but one clinical trials REC reported that they had a dedicated administrative resource; those that had such a resource reported a median of 35 hours administrative support per week.

\section{Stakeholders' perspectives on resourcing of Irish RECs}

In the present study, it was acknowledged that a wide range of levels of resourcing was experienced by different RECs in Ireland:

'Some committees do have good resources. It makes you wonder what committees that don't have good resources are doing ...' (HSE)

The role of support staff, such as administrators, to the ethical review process was identified as a critical resource requirement:

'Certainly one of our group was saying we process a vast amount of material and it couldn't be done without the secretaries, administrators, e-mails flying around the place. Think of all the other work backstage that must continue, that must be supported and ... must be resourced.' (HSE)

Participants recognised that a number of issues related to the assessment of the adequacy of resourcing for REC functioning, such as the organisation of work and workload:

'The resources that are available for the committees themselves both the human and the financial resources ... It was thought that where they [resources] exist and where people's [work for the committee is] kind of planned into people's work time, it seemed to be developed better. And ... it depends on ... the volume of the activity within the organisation, how robust the processes are.' (HSE)

\section{REC training}

The ethics review process requires a certain degree of specialist ethical expertise. Chairpersons, administrators and members of Research Ethics Committees (RECS) need to develop the necessary skills to perform their respective roles within the review process. What form this skills development 
takes and what resources are made available to a committee for this purpose often reflects the level of resourcing of the REC, both nationally and in the individual organisations.

While training of REC members is generally considered important (Hanna, 2000; Silberner, 1998) and mandated by some guidelines, in many RECs no specialist training is provided; applicants acquire the necessary expertise through participating in committee deliberations and 'learning on the job'. In many cases, there exist extensive national or institutional guidelines for research ethics review that provide information and guidance on core ethical issues and/or procedural aspects of the review process. REC members might have to become familiar with a range of regulations and statutory requirements, and acquire an extensive specialist knowledge base.

If training is provided to REC members, it often takes the form of workshops, where participants reflect on and discuss relevant case studies in a group format by simulating REC deliberations. An alternative, relatively common format for REC training are online training materials; although these lack the interpersonal dimension, they can provide structure and guidance to REC members in their acquisition of relevant knowledge. In countries with large numbers of RECs, dedicated textbooks have become available for similar purposes. In some countries, induction programmes or continuing education programmes are mandatory for REC members and offered by a range of organisations. In the UK, for example, requirements for induction and ongoing training have been spelt out, but there remains a certain degree of flexibility in terms of training requirements; self-study and informal inductions are considered sufficient under some circumstances (see http://events.npsa. nhs.uk/nres). Delivery of training beyond a comprehensive induction pack is not exclusively the task of the NRES and is offered by independent organisations, such as AREC (see www.arec.org.uk), Professional Ethics at Keele University (PEAK) (see www.keele.ac.uk/depts/pk/ret.html) or the ERSC (ESRC, 2007a and 2007b). Particular attention is sometimes paid to the educational needs of community or lay members (PRE, 2007) and in some cases dedicated training material has been developed to meet their specific needs (Rivera et al, 2004).

Administrators of committees may receive a specific training programme for the tasks involved in REC administration. In the UK, the NRES provides induction and ongoing training for its REC Coordinators (see www.nres.npsa.nhs.uk/rec-community/tools-for-rec-coordinators). In the USA, an accredited professional programme has become available in recent years to train Certified IRB Professionals (CIPs), which is directed at REC members and administrators (see www.primr.org/ Certification.aspx?id=206); this programme is overseen by the Council for Certification of IRB Professionals (CCIP) (see www.ptcny.com/clients/CCIP).

Training in research ethics is not just important for REC members and administrators, but also for researchers and other stakeholders in the research ethics process. It has been pointed out that, so far, research ethics training for researchers, especially during their academic research training, has been underemphasised (Faden et al, 2002; Gordon and Parsi, 2002; Rhodes, 2002; Roberts et al, 2005; Sharp, 2002), even though researchers themselves see a demand for such training (Roberts and McAuliffe, 2006). Training is frequently restricted to the online availability of relevant guidelines. Dedicated distance learning research ethics training materials, especially online tutorials, are made available in some places by national research ethics governance bodies, research funding bodies or individual institutions. Some helpful tutorials include a PRE tutorial on the Canadian Tri-Council Policy Statement (see http://pre.ethics.gc.ca/english/tutorial/survey.cfm), a research ethics training programme offered by the NIH Office of Extramural Research (see http:// phrp.nihtraining.com/users/login.php, from 1 March 2008), a research ethics tutorial by the NIH for NIH staff (see http://researchethics.od.nih.gov), a tutorial that was developed for the US Office for Research Integrity in 2002 (see http://ori.dhhs.gov/education/products/montana_round1/ research_ethics.html) and tutorials in research ethics specifically directed at scientists (FHI, 2001). In the USA, some certified training programmes are available for researchers with different functions; however, the extent of the specifically ethical elements contained in this training are somewhat unclear (see www.primr.org/uploadedFiles/PRIMR_Site_Home/Certification/CIP/Navy\%20 $-\% 206.2007 . p d f)$. However, the availability of research ethics learning and reflection opportunities 
beyond compliance-focused training in guidelines, which some consider to be essential for achieving a good quality of research ethics practice (Faden et al, 2002; Guillemin and Gillam, 2004; Loff and Black, 2004; Taylor, 2007b), is frequently left to individual institutions, which may or may not provide such additional learning opportunities.

\section{REC training in Ireland}

Based on the survey data, the provision of dedicated research ethics training for members of Irish RECs is not the norm. While $44 \%$ of surveyed RECs stated that they provided training for their members, only $13 \%$ of them had a dedicated budget for training. The types of training delivered were not specified by the majority of committees surveyed and their appropriateness can therefore not be assessed. Among the types of training that were explicitly mentioned were outside training courses, conferences, guest speakers and introductory seminars. While stakeholders are not unanimous in considering lack of training as problematic for the functioning of RECs in Ireland, the lack of training and the wish for better training opportunities were both topics frequently raised in interviews by stakeholders.

\section{Stakeholders' perspectives on REC training in Ireland}

In the present study, there was great variation among RECs, and indeed among individual members, as to the extent and form of training received. The variation in training ranged from 'none at all' to 'ongoing external expert training'. The RECs that offered no formal training appear to hold the expectation that as contributors to the research field, members bring the requisite ethical knowledge with them:

'I guess there should be some formal training, but there isn't here. It's taken ... if you're an academic member of staff ... you're expected to do research in the area [and] ... you would be ... conversant with the ethics principles of the professional body.' (AC)

The contribution of members with specific expertise to act as a form of training was considered by a Chair of one academic REC:

'I think we train each other in the sense [that] ... we have people with particular expertise working in the biomedical area, in the children's area and disability area, etc. So I think we train each other and we haven't any current plans to ... establish a more formal training programme.' (AC)

A few RECs offered more formal training through, for example, a series of seminars or workshops, some open to the wider research community, on various topics relating to the ethics of research:

'We do conduct seminars and bring speakers, experts in the field, into the college. We have done a number of these exercises and will continue to do so.' (AC)

Other RECs drew on specific organisations with research ethics expertise:

'We have organised [training] through SCOPE actually and with the Council for Bioethics.' (A)

Several RECs had undertaken the training offered by Keele University in the UK:

'Currently, several Research Ethics Committees underwent training provided by the Centre for Professional Ethics at Keele University in the UK, a body who have a good reputation here.' (AC)

Administrative staff had generally attended some formal training in various forms. A number of administrative staff referred explicitly to Good Clinical Practice (GCP), which is an international ethical and scientific quality standard for designing, conducting, recording and reporting trials that involve human subjects:

'I was just developing it myself, going to courses as they came up. The GCP days, if there was anything out there. I went to a few conferences.' (A) 
Although this was not the case for all administrators, as one stated:

'In recent times, there has been some informal efforts ... by my counterparts in other hospitals and ourselves. We get together from time to time ... in a gathering that's more informal than formal, and we exchange views on things and we seek one another's advice and opinions about treatments going on. In that way, I suppose there is a feeling of peer support out there, which isn't as much as training but perhaps it's an approach to training.' (A)

Researchers recognised their own training needs:

'All would benefit from training and support in this area.' (CR)

Committee members were also cognisant of continuing training requirements:

'I think with ethical issues, they are all the time changing, growing and developing, and ongoing training is required. Every piece of research I look at, there is another issue that I might not have considered before.' (CM)

However, difficulties with training were also identified, particularly by Chairs in hospital RECs in relation to attendance:

'It is genuinely hard ... as we have three busy surgeons, that you have to go off and do a training day for ethics.' (HC)

Another concern involved what training actually constituted:

'What is the curriculum? Training is training of what? There is no point. We are not sending them off to learn about Kant and Mill. That isn't going to happen ... It is knowing what do you want to teach them.' (HC)

This issue was addressed at the HSE Consultation Day by one participant:

'We suggested that there should be a national standard for training, developed in conjunction with the Bioethics [Council] ... also with the HSE and HIQA [Health Information and Quality Authority] and any other interested bodies, so that there is a national standard around this ... we thought this should be nationally devised with input from all the local research ethics committees themselves.' (HSE)

The need for training was acknowledged, especially for those in positions of particular responsibility such as REC administrators and chairpersons:

'Well, I think ... if you want to start somewhere, it has to be training the officers, the Chairman and the Vice-Chairman. It makes perfect sense that they at least should be brought up to speed on what is required.' (HC)

\section{Ethics Review Process}

\section{Standard operating procedures and application materials}

Most committees work on the basis of standard operating procedures (SOPs) that state the organisational framework and the procedures according to which the REC operates. Such standard operating procedures can be developed by each individual institution based on general guidelines (CIHR et al, 1998/2005; HHS, 2005; ICB, 2004; NHMRC, 2007) or might be provided by an overarching body (NRES, 2007; NZHDEC, 2006). Most RECs require that applicants fill in an application form. Often these forms are developed by individual committees. However, there are REC systems that rely on the use of a centralised national application form (e.g. in the UK, Australia, New Zealand and Denmark). In other systems, such common application forms might be adopted by sub-units of the REC system, for example, by large funding bodies or regional bodies. 
Proponents of the development of local application forms argue that there is a need to reflect the concerns encountered in their particular area of practice and to be sensitive to the needs of researchers in the particular research field. In some places, there may be different application forms for different areas of research, such as quantitative and qualitative studies. Proponents of standardised application forms, on the other hand, argue that standardisation is ultimately more researcher-friendly, insofar as the review process then becomes predictable for everybody concerned, which can facilitate REC members as well as researchers. It is claimed that the majority of the available standardised application forms adapt the application pathways depending on the type of research that is reviewed (e.g. the NEAF Quick Reference Map, see www.neaf.gov.au). Separate application forms are frequently in place for those types of research that are eligible for exemptions or expedited review procedures. Most research ethics guidelines allow for this occurrence for certain types of low-risk research (CIHR et al, 1998/2005; HHS, 2005; ICB, 2004; NHMRC, 2007; WHO, 2000), based on a screening process that establishes whether applications count as no or very low risk. However, the reliability and validity of such a screening process, which often depends on the judgement of just one individual, has been questioned (Elliott and Hunter, 2008; Hunter, 2007b).

In addition to the application form, there is usually a requirement for the inclusion of supporting documentation, comprised most frequently of a more extensive project description, sample recruitment material (e.g. contact letters), participant information sheets and consent forms for each participant group. In clinical trials applications, extensive additional information, including safety monitoring documents, needs to be submitted. Application for a non-invasive study, for example, easily exceeds 40 pages; application for a clinical trial requires significantly more extensive paperwork. In addition, many RECs require that a number of hard copies be submitted, although recent modifications towards a comprehensive and integrated online application system in some jurisdictions have reduced that burden.

\section{Stakeholders' perspectives on standard operating procedures and application procedures}

\section{Survey data}

Online submission is facilitated by only 3 RECs in Ireland, but only 9 report that e-mail submission is not possible. All allow submission by hard copy. While over $90 \%$ of RECs surveyed report that they have a standard application form, only $68 \%$ of them operate on the basis of standard operating procedures. Most of these RECS made their standard operating procedures available to the research team.

In relation to review procedures, $74 \%$ of RECs surveyed facilitate expedited review, i.e. review modes other than by the entire committee. This 'fast track' method is generally employed for low-risk research (i.e. not with potentially vulnerable populations or involving any physical or psychological risk to participants) or amendments to original proposals. Regarding the average timeframe between submission of research proposals and REC response, first feedback to applicants is achieved in a mean of 21.8 days (s.d. $=10.8$ ).

According to survey data, and in keeping with international experience, the vast majority of submitted research proposals are ultimately granted approval by the RECs. However, if a submission is refused, $66 \%$ of RECs state that they give the researchers the option to appeal the decision. No data were available on the specific characteristics of appeals procedures and their uptake by researchers. While the topic was absent from conversations with chairpersons of RECs, researchers frequently described problematic experiences with REC review, which would indicate a need for further attention to be given to the issue of appeals.

In $64 \%$ of cases, approved research is monitored by the REC granting approval, primarily by requesting annual progress reports and a final report from the researchers. This is an obligation in relation to clinical trials since researchers are required to provide information on any adverse events. 
While it was reported that there is a database of REC activity by $55 \%$ of committees, annual reports were produced by only $45 \%$ of the RECs surveyed. In the case of clinical trials, reports are mandatory with regard to a number of factors. For non-clinical trials, it seems from the reports made available to the research team that they can differ considerably with regard to the information included and especially with regard to their public availability. Very few RECs make their annual reports available on their websites.

\section{Stakeholders' perspectives}

The application and review process for ethical approval of research varied across institutions according to the stakeholders who participated in the present study's interviews and focus groups. Hospital-based RECs also varied as to whether they focused on clinical trials or not, and also had different operating procedures for reviewing research applications.

Those RECs that were established for a longer time and were better resourced had a standardised application form and review process. Most of them had a dedicated website, with downloadable application forms and information that assisted applicants and RECs alike. More recently established RECs were sometimes more haphazard in their approach to applications, but often had plans for standardising the procedure:

'I would much prefer it if we had a website where you pulled down a standard application form and everything else.' (AC)

Aspects of the application form itself were viewed as key to the whole approval process:

'There is a page in the ethics application that says, "Are there any ethical concerns raised?" that presents itself as the key page of the application and it's surprising how many people leave it blank because they don't see any issues at all, even when it would be simple “confidentiality"'. (AC)

For RECs without a standardised application form, extra work was involved for both the committee and the researchers, as the following two quotes show respectively:

'You receive different types of documents every time, which in itself adds to your workload in many ways. People take sort of a scatter-gun approach and they'll send you 80 pages, where 6 or 10 might have done. You feel you really have to go through ... [all the pages] in case there might be something that might produce a red flag.' (AC)

'You go in with as much information as you possibly can, even if questionnaires and things [are not ready], just to give them [the REC] an idea of how and what they are ... that just gets the process started ... Then you can get additional approval on changes.' (CR)

Administrative staff, or the Chair of the REC, tended to act as gatekeepers, facilitating researchers and ensuring applications were appropriate and complete before reaching the REC as a body:

'Basically what we do is make sure people send us in the entire proposal or protocol, including in particular the research methodology ... You receive different types of documents every time.' (AC)

'We have also reviewed our documentation numerous times and have tried to make it as simple and straightforward as possible, so that people don't feel that it's another massive form-filling exercise.' (AC)

\section{Review process and results}

Once a study proposal has been submitted, the review process begins. For fast-tracked or expedited review procedures, the application might not reach the full REC, but might be approved instead by the chairperson or a sub-set of committee members. Frequently, such applications may be processed outside the standard timelines of REC review, i.e. will not be dependent on the usual deadlines. However, for each of the review and decision modes, specific quorum requirements need to be set up. For full REC review, it is usually recommended that a certain minimum number of committee 
members be present and that these include a number of non-scientific and community members (CIHR et al, 1998/2005; HHS, 2005; Department of Health and Children, 2004; NRES, 2007; WH0, 2000). Rules are usually in place regarding the management of the not-unusual case where REC members have conflicts of interest; they mostly advise the exclusion of these members from the committee proceedings, but might also require the transfer of the application to another REC (Campbell et al, 2003; HHS, 2005; NRES, 2007; Wolf and Zandecki, 2007). The model of decisionmaking employed is most frequently a consensus-based model rather than a majority model. This is not just explicitly advised by some guidelines (CIHR et al, 1998/2005; NRES, 2007), but also broadly accepted as preferable in the REC community despite some concerns about potential pitfalls arising in the consensus process (Loff and Black, 2004; Moreno, 1995; Spielman, 2003).

There are different models for the review of proposals in RECs. Some provide every member with full documentation of all proposals that go through the committee and require them to be familiar with all of them. However, according to some regulations, certain types of information beyond the application form itself need only be made available to members with relevant expertise (NRES, 2007). Many committees work according to a 'lead reviewer' system, whereby each proposal is assigned to a sub-set of members who have the responsibility to review the application thoroughly and report to the committee, which then bases its deliberations on the report of these lead reviewers (NRES, 2007). In some RECs, the Principal Investigator or a member of the research team attends the REC meeting so as to be available for questions and clarifications; such attendance where envisaged is more often optional than obligatory (NRES, 2007).

After consideration of the application, the REC comes to a decision on how to proceed. Usually, its options include some version of the following (sometimes 'deferral' is not given as a separate option, but falls either under 2. or 4.) (ICB, 2004; NRES, 2007; NZHDEC, 2006):

1. Approval.

2. Provisional approval that is subject to conditions, i.e. pending minor modifications.

3. Deferral and re-submission, pending major modifications.

4. Rejection.

In most RECs, the majority of proposals receive provisional approval. Outright approval is less frequent; deferral and re-submission are even less common, and outright rejection is comparatively rare. These judgements have implications on the timeline for completion of the research. Usually, re-submissions are more time-consuming insofar as they usually require a renewed full ethics review, including the standard timelines. In the case of provisional approval, there usually is a fasttracking system in place that allows the researcher to make modifications and submit them without being subject to full REC review again. Despite this abbreviated additional process, it may still add significant extra time to the review process, especially in cases where proposals have to go through several RECs that might require different modifications, which then need to be coordinated by the researcher and can require extensive negotiations with different committees.

\section{Stakeholders' perspectives on review processes and results}

In the present study, there were many procedural differences in the review process after receipt of the application for ethical approval. Some RECs operated a pre-ordained policy of review by Chair only:

'Not every study has to go to a meeting of the committee. Some are amenable to what we call Chairman's action.' (A)

In some RECs, for specific types of request for ethical review, mechanisms were put in place to expedite ethical approval. For example:

'We agreed in our first meeting that we would establish a fast-track process, whereby any application or any request for approval that is time-critical, usually by virtue of a funding application, is reviewed by [the Chairperson and] by a member of the committee.' (AC)

'Certainly not the clinical trials, but a lot of minor stuff that doesn't seem to involve a lot of major ethical problems, any risk or danger of injury to anybody, for example, questionnaires and the like, I would tend to do them and the committee has mandated me and authorised me to give Chairman's expedited approval. And that seems reasonable.' (HC) 
A call for expedited review of other forms of application was proposed at the HSE Consultation Day: 'If you want to do similar kinds of research to what you have done before, the Ethics Committee would have your documentation on file. You say "I'm doing pretty much the same thing", so that's great - you already had ethical approval to do it before. We should be able to expedite the procedure ... it would streamline the thing enormously.' (HSE)

The decision as to whether an application for ethical review is expedited or not is generally taken by the Chairperson of the REC:

'I do the screening. There's no point in swamping the committee with this.' (HC)

or by an administrator:

'... just based on our [administrator's] experience, we can make a fair guess at which studies are for the Chairman and which studies are for the Committee.' (A)

There was little consistency between stakeholders reporting on the manner in which the review process was undertaken by the RECs. In some committees, each application was reviewed by all committee members before being discussed by the group:

'Send the combined comments to everybody for the meeting ... and then all the comments are put together in one document and sent out to everybody before the meeting, so that when you come to the meeting you have a document that has the reviewers' comments.' $(\mathrm{AC})$

In other RECs, a sub-committee of two or three, usually including the Chair, reviewed individual applications and reported back to the main committee for approval or for discussion if issues arose: 'We don't send all the paperwork to every committee member. We select some essential material of the application form, the patient information leaflet, perhaps one or two other documents that are not too voluminous, and we copy these and send them to every committee member. And then the rest of the material we give to one member of the committee or one of the expert members, and we ask them to review that in detail. And then on the day of the committee meeting, they come to the meeting armed with all this paperwork and they give a report to the meeting on their review. And in that way, the committee is informed of the total submission. And on that basis, the decision is then made.' (A)

Once an application has been ethically reviewed and conditionally approved, the Chair of some RECs will then ensure that what the committee asked for is undertaken and then grant approval on that basis.

Little reference was made to any appeals process, but one Chair stated:

'I guess the only appeals process is that people can come back to the committee ... via e-mail or e-mail through the Chair. Or actually, we do occasionally allow applicants ... to make a case before the committee.' (AC)

Reference was made to the specific challenges of the ethical review process for multi-centre research:

'If you're doing work at a multi-centre level, that is problematic. We're not proposing the solutions at the moment, but it is problematic if you are trying to do work at a countrylevel, particularly involving all hospitals. The procedures are not simple.' (HSE)

One researcher referred to their own experience of undertaking a multi-centre study, which clearly demonstrates the difficulties and complexity of having to apply to all committees separately: Researcher: 'It has gone through the ethics committee in one adult hospital in [Organisation A] and in two children's hospitals in [Organisations B and C], and then another adult hospital totally changed the format of what I had to do.' (CR)

Interviewer: 'Requiring a different protocol?'

Researcher: 'A different protocol, a different procedure.' (CR) 
Clinical trials research has negated this difficulty according to the Chair of one hospital REC:

'Clinical trials legislation has worked in that regard. The fact that the researcher does not have to go to Dublin, Cork, Galway and every single one [REC] has actually worked. And that has reduced the workload ... It is very very welcome indeed.' (HC)

The reason for the current need for multiple REC review was articulated by one Chair of a hospital REC:

'To an extent, we are all not sure where the boundaries exist between us.' (HC)

\section{Record-keeping and monitoring}

Research Ethics Committees (RECs) usually keep a record of the proposals that they review. In some jurisdictions, detailed instruction is given on what records are required (e.g. CFR 46.115 in the USA). However, in jurisdictions where these requirements are less precise, some uncertainty about these is not uncommon and record-keeping is often not handled uniformly: different committees may vary in the details they record and the templates or databases they use. However, data that are usually kept include the applicant's details, proposal title, review result, turnaround times, updates and final report; the application form and supporting documentation may also be kept on record.

After a proposal has been processed and approved, most research ethics guidelines require researchers to submit updates on the research and a final notification or report when the research has been completed. In the case of modifications to proposals (e.g. changes to instruments or methodologies that arise during the process of research), RECs may require to be notified of any changes and conduct a review of these changes, usually through a fast-track system.

One of the concerns is the question of what role the REC has regarding researchers' compliance with the ethical requirements. Even though many guidelines state that researchers should submit yearly progress reports to the REC, or at least final reports after the completion of the project, the precise authority of the committee in this respect frequently remains unclear. In some cases, reports are all that are required. In other cases, RECs are expected to take a more active role in monitoring. The scope and authority of such monitoring or auditing activity is, however, often unclear and over the years it has been debated how effective RECs are in this role and how exactly the role of RECs should be understood (Bankert and Amdur, 2000; Bortolussi and Nicholson, 2002; Faden et al, 1980; Heath, 1979; Saver, 2005). Usually, at least some monitoring and follow-up is the responsibility of the REC (CIHR et al, 1998/2005; ICB, 2004; NHMRC, 2007; NRES, 2007), but under certain circumstances some aspects might be devolved to external committees, bodies or individuals, especially in relation to clinical trials research. The REC's role regarding the monitoring of adverse events in clinical trials (and, specifically in the USA, its relation to an additional type of monitoring body, the Data Monitoring Committees) has been debated (Bankert and Amdur, 2000; Friedman and DeMets, 1981; Gordon et al, 1998; Wells et al, 2000).

One further way to address aspects of the monitoring requirement has been the appointment of individual 'research ethics monitors', either within an institution or independently of institutions. The European Commission is also currently piloting a research ethics monitoring system for research funded under their Framework programmes. A monitoring system is currently also required in clinical trials research in the USA. The function of research ethics monitors is frequently to act as an advocate for participants or patients, and to monitor researchers' compliance with patients' rights requirements (Easa et al, 2005 and 2006). However, according to some models, they might fulfil a broader audit function, looking at the general performance not just of researchers but also the REC. 


\section{Stakeholders' perspectives on record-keeping and monitoring}

In the present study, few stakeholders referred to any monitoring function of RECs beyond acknowledging that it did not occur or was underdeveloped.

'At the moment, there is no monitoring.' (CM)

'We are just trying to establish the procedures ensuring the quality and the ongoing safety and ethicality of studies that have been approved within the healthcare settings.' (A)

'Good monitoring' (HC) was identified as an essential requirement for ethics committees by the Chair of one hospital REC, with the need:

'Entirely for independence and transparency.' (CM)

Those that did refer to monitoring spoke of two distinct types: monitoring of researchers' compliance and evaluation of REC processes. In relation to compliance, participants referred to researchers' adherence to the assertions in their application for ethical approval:

'What they tell us, the data will be in a locked filing cabinet.' (CM)

'How do we know?' (CM)

Reference was also made to the requirement that researchers submit reports on research progress, usually yearly after ethical approval has been granted. While this requirement was said to be stipulated by some RECs, it was not necessarily adhered to. The reason for this was linked by one participant to a lack of REC monitoring:

'On the whole area of monitoring ... one of the biggest problems with most committees is that in the approval letter we send out, stating that we want to see the results ... very rarely do we get the results back. The researchers don't bother because they have finished, so they don't want to bother sending you a copy ... and that I think is a problem. It is a problem of the committee not following it up.' (CM)

There was generally less discussion of the monitoring of REC decision-making processes. However, reference was made to both a proactive audit and reactive policing of decisions:

'In the States, they have separate committees that would actually go and audit, and monitor the committees and monitor how people are applying their ethical approval.' (CM)

'If it's a case that they come across bad practice, for want of a better term, and particularly if it was bad practice by a particular research centre, I think that would be a policing system. That research centre would have to come in and be reviewed.' (CR)

As can be discerned from these quotes, reference to monitoring of processes did not refer to current practices in Irish RECs. Suggestions were made for the development of monitoring of RECs, with the identification of the need for this to be done by an independent body:

'I think that is something that could be done centrally, as in ... [nationally], just like the Irish Medicines Board. That you would have an Irish ethics monitoring committee that would go and accredit ... the individual institutions for the policies that they have in place.' (CM)

It was recognised that the need for monitoring of RECs, at least in clinical settings, may be advanced by external bodies:

'What is coming down the track quite quickly, I gather, is under the FDA [US Food and Drug Administration] regulations, each research protocol has to have a patient advocate appointed ... It's an extensive role and basically the person - they don't call it a patient's advocate, but an ethics advocate or something like that - is responsible to actually interview the participants to make sure that they conform to the ethical guidelines. All the FDA trials need that. That, I think, is coming down the track in Europe, very fast.' (CM) 


\section{Main concerns}

- 72 Irish RECs in operation.

- No broader governance structure in existence, with the exception of EU Regulation for 13 clinical trials RECs.

- Multiplicity of institutional governance models in academic sector.

- Significant differences in resourcing and workloads of Irish RECs.

- Generally increasing review load without increasing resources.

- Lack of standardisation in relation to standard operating procedures, application forms, membership categories and review of multi-centre studies.

- Lack of training and quality assurance measures.

- Recruitment difficulties of Irish RECs regarding lay members and lawyers.

- Strong wish among stakeholders for implementation of central resource for Irish RECs that can provide advice, support, training and networking opportunities. 


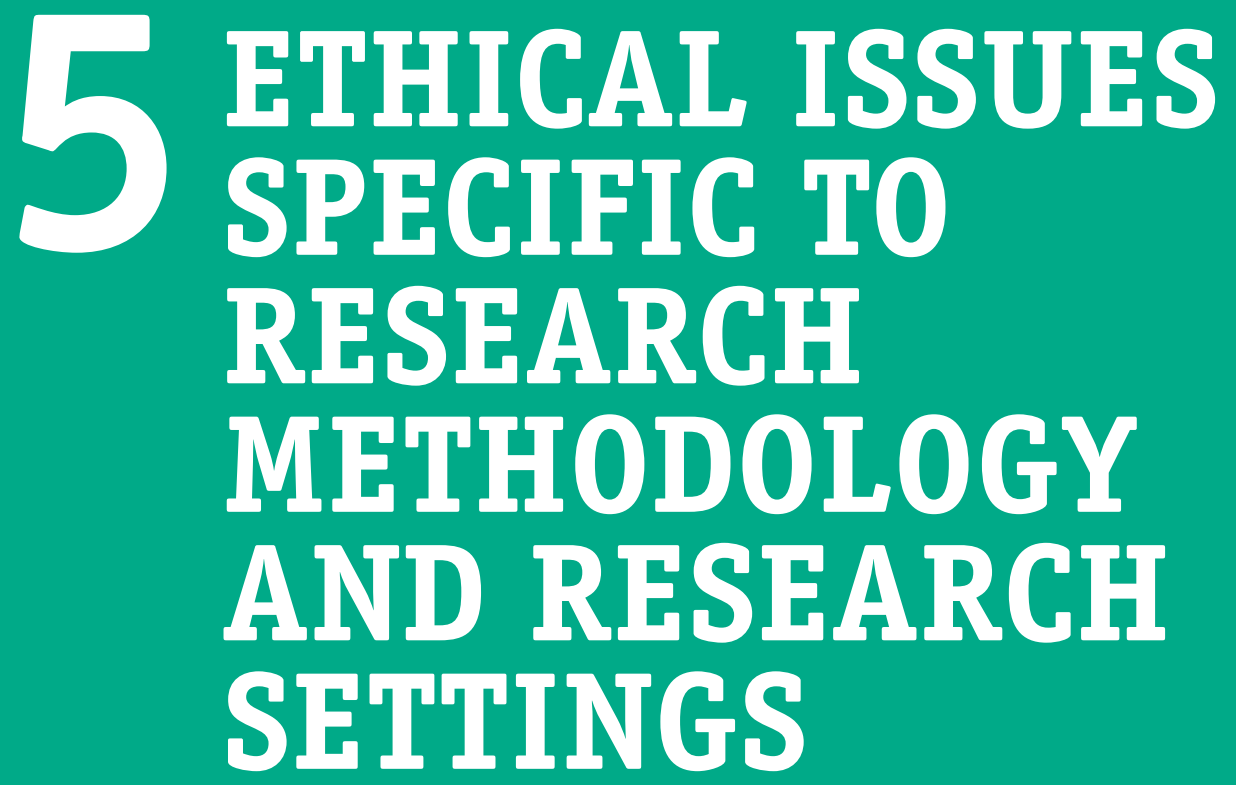


One major concern in research ethics that has been much discussed in recent years is the significance of the research methodology for its research ethics review. Researchers frequently complain that some Research Ethics Committees (RECs) appear unqualified to judge the methodology of their research proposals, leading to needless delays, inadequate requests for modifications or inappropriate rejections of research proposals. Even more broadly, there is concern that the research ethics system has been developed in relation to clinical trials research and has not been adapted sufficiently to do justice to research from other disciplines or research based on different methodologies. In preparing REC applications, researchers are required to adapt their presentation of the research proposal to fit a mould that may be inappropriate for the project in hand. From an ethical point of view, this can easily impair the effective communication on ethical issues between researcher and REC, with the potential consequence of a misunderstanding of the particular nature of ethical challenges encountered in the research.

\section{Ethics, methodology and Social Science and Humanities research}

As already indicated in the discussion on research ethics governance systems (see Chapter 3), there is strong evidence that research ethics governance models are developed primarily with clinical research in mind, specifically trials that follow the randomised control trials (RCT) model. Even within clinical research, different methodological approaches might not fully fit this model and some research ethics systems have published specific guidelines or require additional documentation for different research approaches (NMHCR, 2007, Section 3). Generally, a comparative neglect of social science research is evident and an even more pronounced neglect of research in other disciplines, such as the humanities.

Among the national research ethics guidelines considered here, only the Canadian Tri-Council Policy Statement (TCPS) and the Australian National Health and Medical Research Council (NMHCR) guidelines include more extensive considerations on typical issues arising in qualitative and social science research. In some cases, this focus on clinical research has led to the implementation of research ethics review systems that do not require review of research other than clinical research. However, in many cases a system that was initially developed for clinical research is then stretched to apply to other forms of research. Significant concerns exist in the social science and humanities research communities about the inflexible application of a research ethics system that is not suited to their own research (Allen, 2005; Dingwall, 2007; Ramcharan and Cutcliffe, 2001; Richardson and McMullan, 2007; Van den Hoonaard, 2006a and 2006b). There is some evidence that this has already had a restricting impact on the type of research that is conducted in some social science disciplines (Van den Hoonaard, 2006b).

Literature that is explicitly dedicated to social science research ethics has only been emerging in recent years, even though implicitly many of the concerns mentioned in the literature were taken into account previously (Hesse-Biber and Leavy, 2006). Only quite recently have specific national guidelines been developed for social science and humanities research, and existing general guidelines have been reviewed to include special concerns to be considered in the review of this type of research (Boulton et al, 2004; ESRC, 2006; PRE, 2004). There is an acknowledgement that more needs to be done to communicate and explore the specific ethical challenges that qualitative researchers encounter in their research, not just for the benefit of the research ethics community that is going to adjudicate on the research proposals (Cannella, 2004; Connolly and Reid, 2007; Hemmings, 2006), but also within the researcher community that is planning research projects (Burke, 2007).

While the issue of separate review systems (or at least different application forms for different types of research) has been discussed, such a separation has only been implemented in some places, mostly at the institutional level, for example by introducing discipline, school or facultylevel RECs in academic settings. One common type of REC are specialised committees that 
exclusively review student research within units of academic institutions (CIHR et al, 1998/2005); RECs that only consider research on certain participant groups are another. Alternatively, within a system of less specialised RECs, research ethics application forms might include separate sections on different modes of research.

Issues that have been discussed as problematic in REC review frequently include methodological expectations relating to what constitutes appropriate participant numbers, appropriate instruments and problems with any deviation from an experimental methodology. Frequently, researchers' perceptions are that REC comments on their proposals and demands for revision indicate the REC's lack of familiarity with standards of practice in research that uses non-quantitative methodologies (Lincoln and Tierney, 2004; Owen, 2006). These difficulties become even more pronounced when critical methodologies are employed that challenge fundamental assumptions of the subject matter under consideration (Holland, 2007). Whether a clear distinction between the scientific versus the ethical merit of research proposals is possible has been much debated and is frequently questioned (Dawson, 2005; Dawson and Yentis, 2007). A solution to the problem is sometimes attempted by including a preceding separate step of methodological peer review, but this may not address the underlying problem of the close link between methodological and ethical issues. Unlike some other guidelines that explicitly exclude methodological review from the remit of REC review, Canada and New Zealand include review of methodological validity of the proposal in the tasks of the REC (CIHR et al, 1998/2005; NZHDEC, 2006). Canada's Tri-Council Policy Statement also acknowledges specifically the importance of being aware of specific standards and research approaches in social science and humanities research.

Issues in relation to informed consent are another set of concerns that have received some attention (Wiles et al, 2005). Much of social science research is conducted with healthy volunteers; accordingly, many of the problems in communicating study information, particularly in relation to the therapeutic misconception, are unlikely to occur in social science research. However, research participants can be characterised by other types of vulnerability, which have implications for the informed consent process. Particular concerns arise in relation to the informed consent process in different forms of qualitative research. In many cases, it is not easy to predict the direction that research interactions are going to take (Malone, 2003; Ramcharan and Cutcliffe, 2001). What the content and experiential quality of in-depth interviews will be can often not be fully predicted in advance. There are forms of research, such as participatory research, where essential aspects of the research process and interventions will be successively determined throughout the research process, so that participants can only be provided with information on the initial plans for the general research process, but will not be in a position to consent to a comprehensive and specific set of research interventions. In ethnographic research, for example, which aims at capturing behaviour and interaction in natural settings, the problem of informed consent is particularly difficult to manage. In order to deal with these challenges, the value of extended and reciprocal reflection on the challenges of the process with prospective participants has been highlighted (Etherington, 2007; Harrison et al, 2001; Williamson and Prosser, 2002). It has also been proposed that REC processes might need to be adapted towards an ongoing monitoring process that accompanies the research process (Herrera, 2000; Ramcharan and Cutcliffe, 2001).

Other authors have pointed out that the problem might not be just a practical problem, but one of a more fundamental nature, based on the differences between the standard 'scientific' model, on the one hand, which underlies the REC system and assumes strict hierarchies between researcher and 'research subject', and the participatory models, on the other, which attempt to establish power-sharing between researcher and participant not just in the informed consent process, but throughout the entire research process (Boser, 2007). This has consequences both for the status and content of the informed consent process. The inclusion of highly detailed information on the research project for its entire duration at the time of initial consent is frequently not possible. However, at the same time such consent might be considered to be somewhat less significant than in more traditional research projects since it is not the one and only opportunity that the participant has to make an active decision relating to research participation. Some authors claim 
that some of the standardised demands of the informed consent process, especially the requirement of providing signed informed consent documentation, might alienate potential participants and disrupt the establishment of researchers' trustworthiness instead of ensuring participant protection (Bhattacharya, 2007; Boser, 2007; Harrison et al, 2001).

Another ethical issue that arises in relation to social science and humanities research includes the different types and levels of risk that participants encounter in the research. Points of view differ considerably with regard to one of the most frequently used qualitative methods - in-depth interviews with participants. Some argue that this methodology allows participants significant control and does not represent a risk over and above the risks encountered in their daily lives (Corbin and Morse, 2003). Others highlight the risk that participants might be encouraged to explore difficult issues with a stranger without the benefit of longer term availability of emotional supports. Accordingly, researchers' responsibilities include careful consideration of the potential for emotional harm and pre-planning of relevant support services should problems arise.

In the context of particular types of risk, one type of concern that has been brought up is research with participants to whom a previous relationship exists. It is not uncommon for qualitative research to draw on respondents with whom the researcher has pre-existing or simultaneous personal or professional relationships, or to take place in settings that the researcher is familiar with through personal or professional experience. Under these circumstances, the management of roles and boundaries and the potential for role conflict becomes a prominent ethical issue (Tickle, 2001). The merging of different roles might lead to misunderstandings and incorrect expectations by participants regarding the responsibilities and attitudes of the researcher in relation to their research activities. These might either lead to feelings of betrayal on the participants' side if researchers keep their role separate and do not fulfil these expectations, or alternatively they might compromise the research process if the researcher is unsuccessful in managing the different role expectations. Boundary issues are rarely discussed in research ethics over and above the issue of researchers' power, but are a significant ethical topic in the area of social and psychological services. However, while these problems are more likely to occur in qualitative research, it should be kept in mind that even though boundary issues are comparatively insignificant in the majority of once-off experimental research, they can be quite significant in those types of clinical research where the researcher is also in charge of treatment.

An additional problem regarding risk and benefit can occur in those kinds of social science research that have a critical orientation towards their research subject (e.g. sociological or ethnographic work on organisations, public decision-makers). As, for example, the Canadian Tri-Council Policy Statement recognises (p. 15), such critical research can be valuable and legitimate, even though it may not rely on collaboration with the research subjects and might not have a positive harmbenefit balance for these subjects. In that kind of research, it might also not be advisable, under some circumstances, to share research results with research subjects (Cooper, 2007).

With regard to specific research methodologies, naturalistic observation and ethnographic methods are particularly prone to encountering problems in research ethics review. The problem of traditional research ethics review for research proposals using ethnographic methodologies has been pointed out repeatedly by researchers (Bosk, 2007; Cooper, 2007; Herrera, 2000; Marzano, 2007; Tolich and Fitzgerald, 2006). The problems encountered in the review process are even more pronounced in so-called auto-ethnographic research, where the researcher is also the research subject and writing is characterised by a high degree of subjectivity (Rambo, 2007). One of the main ethical problems for ethnographic research is the requirement of achieving participants' informed consent. Especially in fieldwork in larger institutions where the number of observed participants is large, it may not only be difficult to receive informed consent from everybody observed, but it could also be detrimental to the validity of the research if it addresses sensitive or problematic aspects that might lead to participants modifying their behaviour (Marzano, 2007). 
Another significant issue in this context is the issue of anonymity, which might be challenging to fully preserve in ethnographic research of well-known institutions or settings with unique characteristics (Tickle, 2001; Van den Hoonaard, 2003). The Canadian Tri-Council Policy Statement is one of the few ethical guidelines that addresses the problems of this kind of research: it states explicitly that it should not be disadvantaged in research ethics review and generally be considered low-risk research, subject to addressing the issue of identification and anonymity in an effective manner (CIHR et al, 1998/2005).

Another research approach that is frequently identified as encountering difficulties in traditional 'regulatory' research ethics review is participatory and action research (Bhattacharya, 2007; Blake, 2007; Boser, 2007; Burke, 2007; Cahill et al, 2007; Martin, 2007; Owen, 2006; Shore, 2007; Williamson and Prosser, 2002). In addition to the previously addressed challenge of a significantly higher degree of unpredictability regarding the research process, which has an effect on the informed consent process, it also brings up the issue of the definition of the role of both researchers and participants. With regard to researchers, the role of participant advocate is sometimes adopted in the process, and in some types of action research this may lead to conflicts with the role of researcher (Tickle, 2001). With regard to the role of participants, it is not uncommon in such projects to consider them to be co-researchers, which then brings up issues of role definition and the delineation of responsibilities as participants and researchers. In negotiation with participants before and during the research project, different understandings on what constitutes ethical practice might emerge, which might lead to conflict with standard understanding of research ethics requirements (Bradley, 2007; Cahill, 2007; Elwood, 2007; Hopkins, 2007; Prilleltensky et al, 1996). Dealing with these differences without privileging the researcher's and REC's viewpoint is a significant ethical challenge for participatory research, which increases in significance the further removed the researcher's culture and experience is from that of the community (Myser, 2004; Sultana, 2007).

One further issue in this context is the role of the community in research. Many participatory and action research projects are conceptualised as community-based; however, the precise format of the involvement of the community can raise ethical issues. Most frequently, RECs consider the issue of community involvement for the informed consent process. This is not just restricted to community-based research in the strict sense, but is a significant general issue in research with those minority groups that rely on collective decision-making. Several national guidelines highlight the importance of community consultation mechanisms, for example, for research involving Canadian Inuit and other Aboriginal peoples (CIHR et al, 1998/2005), New Zealand Maori (NZHDEC, 2006) and Australian Aboriginal and Torres Strait Islander peoples (NHMRC, 2003a and 2007). However, community involvement is an equally significant issue for any research that takes place within a community or has the goal to have an impact on aspects of community life or community health (Green and Mercer, 2001; Khanlou and Peter, 2005). Such involvement requires the identification, or even creation, of appropriate consultation partners and consultation mechanisms, such as Community Advisory Boards, Independent Community IRBs or Environmental Health and Community Review Boards (CCPH, 2007; Steven, 2006). However, the selection of consultation partners or consultation mechanisms might itself turn out to be ethically challenging (Berg et al, 2007; Minkler et al, 2002). Finally, the question of feeding back research results and finding ways of involving the community in the implications of the research are other significant concerns (Macaulay et al, 2007).

Debates on research ethics in social science and humanities research have also highlighted difficulties relating to research ethics in those disciplines where there is no tradition of research ethics review. The perception of many authors in these areas is that RECs often approach research in these subjects with an insufficient understanding of the specific nature of their research, which can lead to requirements that endanger the very nature of quality work in a range of disciplines or specific research areas, such as language studies (Fox et al, 2006), oral history (Janovicek, 2006), journalism (Dash, 2007), Internet research (Kitchin, 2003) and education (Hemmings, 2006; Owen, 2006). 


\section{Ethical issues in relation to methodology and settings for children's research}

The general difficulties encountered regarding ethics review of qualitative research are further compounded by the perception that children are vulnerable subjects who need to receive a particularly high level of protection. If the research ethics review is guided by the suspicion that qualitative methods are less scientifically valid or less controllable in terms of the impact they may have on children, this can have consequences on the willingness of RECs to approve them. A general characteristic of conducting research with children that can present a challenge in the ethics review process is the potentially more unstructured and less predictable nature of the research activities and the relationship between researcher and child (Cummins, 2006; Mishna et al, 2004), as well as the demand for researchers' flexibility in how the child is involved in data collection, which researchers have argued is essential to ensure a good quality of response in qualitative research with children (Bushin, 2007; Coyne, 1998). Such flexibility might be considered a problem if ethics review operates under the assumption that data collection needs to fulfil standardisation requirements akin to those in quantitative research with adults.

As already indicated with regard to adult research, participatory research frequently encounters difficulties in ethical review, even if conducted with adult participants, because its methodological assumptions are relatively far removed from the traditional experimental paradigm. In the case of research with children, who are perceived as a particularly vulnerable group, there might be even less willingness to allow research that employs such methodologies. The comparatively vague role of the researcher in participatory research might also be one reason for male researchers' experience that in conducting research in children's natural environments, they frequently encounter a high level of suspicion and gender-based problems of access (Barker and Smith, 2001; Horton, 2001). On the other hand, as researchers who employ such participatory methodologies have pointed out and as has become increasingly more accepted in recent years, such work can be considered to have a particularly positive ethical value and to ensure that children's rights are taken seriously in the research process, insofar as it promises to remedy the problem of children's comparative powerlessness in the research situation (John, 2007; Morrow and Richards, 1996; Valentine, 1999).

It has been pointed out by researchers that children are generally not used to being taken seriously by adults and can perceive themselves as powerless vis-à-vis adults. The realisation of participatory methods is one way of treating the child as an expert or 'co-researcher', thereby addressing and potentially overcoming this power imbalance in the researcher-child relationship (Christensen and Prout, 2002; Holt, 2004; McLaughlin, 2005 and 2007; Punch, 2002; Smith et al, 2002; Thomas and 0'Kane, 1998). However, it is important that researchers take the co-researcher role seriously and pay attention to their own responsibilities towards facilitating this role and the potential ways in which they might still influence, and potentially distort, children's contributions to the research. Researchers also need to be attentive to the children's specific competencies and the modes in which they may best express themselves to facilitate meaningful participation (Morrow and Richards, 1996). This includes indirect expressions of children's wishes to stop participating. Generally, it is important to be aware that facilitating children in the role of co-researchers is demanding in terms of research design and in terms of input and engagement expected from children; its value and feasibility in each individual case needs to be carefully justified.

Other issues to keep in mind are the various social factors and complexities of the situation in which the research takes place and which might have an impact on the relationship construction between researcher and children (Holt, 2004; Huber and Clandinin, 2002; McDowell, 2001). In participatory research, children may be encouraged to contribute to developing ethical criteria for the research (Flicker and Guta, 2008), setting the agenda for and during data collection, and interpreting results (Thomas and O'Kane, 1998). However, potential limitations of an approach that relies strongly on children's own research interpretation have been pointed out (Punch, 2002). An essential part of participatory approaches is the use of some form of community engagement and consultation (Fisher, 2003a; Fisher and Wallace, 2000; Flicker and Guta, 2008; Helgeland, 2005; 
Tayler et al, 2005). While community involvement can be truly empowering, it is also possible that its value can be undermined either by exploitative use of young people in public fora (Allard, 1996) or by formalistic interpretations of research ethics requirements (Martin and Lantos, 2005).

Specific ethical issues can arise not just due to methodological concerns, but also in relation to research settings. Research in non-traditional research settings (e.g. outside controlled university or laboratory settings) is relatively common in the field of children's research, where particular importance is often given to encountering and observing children in their natural environments, such as at schools, in day care centres, in playgrounds or at home. In these settings, observational methods are frequently employed. Direct observation can offer an important window into children's lives, especially in research with smaller children who may not be able to verbalise their experiences. Observations in group or public settings raise issues of informed consent when some of those present have not given valid informed consent, as, for example, by forgetting to submit the informed consent forms (McKechnie, 2006). Another concern in relation to observational studies with children is the issue of the role of the observer and the question whether there are circumstances when researchers should intervene in what is happening. Problematic behaviour that might call for intervention could, for example, include the observation of bullying, racism, exclusion or other types of aggression (Barker and Smith, 2001; MacNaughton and Smith, 2005). In order to circumvent such problems, some researchers choose to develop experimental alternatives to observation in naturalistic settings (Underwood, 2005).

Particular challenges are encountered in school-based research or research in other educational settings. Guidelines and publications by educational research associations address some issues in educational research (BERA, 2004; Strike et al, 2002). However, it is still not common in educational research to explicitly explore ethical issues in research and unexpected complexities are likely to arise in research practice (Kennedy, 2006). Educational research is sometimes given a particular standing regarding research ethics; for example, in the USA, research that only involves 'normal educational practices' has traditionally been considered exempt from review according to CFR 46.101b1 (HHS, 2005). However, some school-based research might be concerned less with normal educational practice and employ social research methodologies. It has been argued that in these cases, research should undergo ethics review; however, it should be expected that the membership of RECs includes people with educational knowledge (Howe and Dougherty, 1993; Howe and Moses, 1999).

One issue regarding all school-based research is the particular approach that should be taken to gaining consent for research participation. Obtaining consent for school-based research faces particular challenges because of the involvement of a range of different stakeholders, including school boards, Principals, teachers, parents and children (Valentine, 1999). There is currently no international consensus on best practice in this context regarding the precise role of these stakeholders. School boards and Principals are the primary gatekeepers for research in schools, with Principals usually being the primary decision-makers regarding access. However, in some places such as Canada, an additional review process might be added, whereby dedicated district school board RECs become an additional gatekeeper by reviewing a research project and deciding whether it merits access to school populations in their jurisdiction. Given the prominent role of negotiations between gatekeepers and researchers when determining access to children, decisions might inadvertently lead to neglecting the role of the child in the informed consent process (Heath et al, 2007; Homan, 2001).

Some schools or institutions may not themselves require a particular approach to receiving parental consent and may leave the decision on whether to contact parents up to the researchers (David et al, 2001). While requiring active written parental consent ('opt-in') for children's participation in school-based research is often considered ethically most appropriate in the research ethics community, sometimes 'passive' or 'opt-out' consent is chosen instead (Santelli and Smith Rogers, 2002). This means that children participate in the research project unless they or their parents actively object. Advantages of opt-in consent are ensuring awareness and involvement of parents in the research process and the possibility for parents to actively address the issue 
with their children (Moolchan and Mermelstein, 2002). Disadvantages of opt-in consent are the higher resource-intensiveness and the potentially lower response rate. While there is evidence that these challenges can be manageable for some projects (O'Donnell et al, 1997), researchers frequently report concerns, especially about sample bias effects in opt-in consent (Moolchan and Mermelstein, 2002). In Australia, the national guidelines include provision for the possibility of 'standing parental consent', whereby parents delegate decision-making authority to the school for participation of children in certain low-risk observational or questionnaire studies (NHMRC, 2007). Excluded from 'standing parental consent' would be any study with children who are not considered mature adolescents that addresses either non-beneficial research or research on 'subject matters involving sensitive personal information or personal and family relationships' (ibid, Paragraph 4.2.11). In relation to informed consent, school-based research can also face ethical challenges if a research project is related to the implementation of a mandatory programme where issues of informed consent and voluntariness arise, as, for example, a programme for mandatory drug-testing for school athletes (Shamoo and Moreno, 2004).

In addition to the realisation of informed consent, some researchers have highlighted additional issues regarding the relationship of the researcher to the students they intend to research. Researchers seek access to students for a variety of reasons:

- The school setting is chosen merely because of convenience for research by outside researchers who require access to children as a research population.

- The setting is essential to the research question and the researcher is an outsider with no pre-existing relationship to the research population.

- The setting is essential to the research question and the researcher has a pre-existing function in the school, where this function may or may not be essential to the realisation of the research question.

When researchers are outsiders, questions relating to what justifies granting them access to the school setting arise, especially in the case of research that takes place during ordinary class time and might disrupt ordinary school routine. Within the school setting, which is governed by hierarchies, discipline and norms of conformity, the issue of the researcher's role definition and power becomes particularly pertinent. This will have a significant effect on the meaning of the informed consent process for students; there is evidence that students might perceive participation in research during class hours as another educational experience or 'just another piece of schoolwork', rather than something that they see as independent from the norms that govern the school context, especially if research activities have features that resemble educational activities (David et al, 2001; Denscombe and Aubrook, 1992; Edwards and Alldred, 1999). More personalised effects of power can be expected in research involving staff members, whose non-research role also gives them formal disciplinary authority vis-à-vis their pupils. Additional issues arise if research is a compulsory part of the teacher's training (Howe and Moses, 1999) or if teachers themselves become research participants, as, for example, in research projects on professional development (Tickle, 2001). Similarly, researchers who are present over an extended period of time and become involved in relationships with staff, students and parents will face more complex role-related problems.

School-based research also raises the issue of ethical implications of conducting research in group settings. Children are either involved in group activities, like focus groups, or research is at least physically conducted in group settings, for example, when children fill in questionnaires in the classroom. One potential risk in these circumstances is that research activities might place children at risk of negative reactions from others in the group. Negative effects may be due to something that children contribute as part of the research activities. Some research areas seem to bear particular potential for negative responses, such as research on social relationships and popularity, even though it has been reported that there is little empirical evidence of negative effects of quite risky seeming socio-metric research (Mayeux et al, 2007). It is important for researchers to be careful when eliciting children's contributions and to remind children that they can remain silent rather than talk about uncomfortable issues in front of other children. 
Another potentially difficult situation is children's exclusion from research activities if they or their parents opt out of participating in the research. It is important to offer suitable alternatives to those not participating so that they do not feel excluded or be perceived as excluded by their classmates. However, in many cases the researcher will not be in a position to clearly grasp potentially problematic dynamics in the group and will need to make sure that some arrangement is in place to deal with matters that arise from the research, but that may only become obvious after its completion. In these cases, arrangements for detecting and addressing potential negative consequences in the school context (or equally in other institutional contexts where research is conducted) need to be put in place.

Ethical difficulties gain a further level of complexity when research takes place in the children's family home, an approach taken especially in research with smaller children. The home setting raises a number of challenges that have been addressed by researchers (Bushin, 2007; Nilsen and Rogers, 2005; Valentine, 1999). One of these is privacy, which is significant in different ways. First of all, the researcher enters the private realm of the child and their family, which might be perceived as an intrusion (Valentine, 1999). It is likely that the researcher will learn a significant amount about the family's circumstances and everyday life over and above the stated purpose of the research. Another concern is related to the researcher's attempt to facilitate privacy for the research conversation with the child, without becoming open to allegations of abuse, as, for example, if they spend time alone with the child in their bedroom (Barker and Smith, 2001). In any case, the complexity of the researcher's situation in the home setting should not be underestimated: the researcher encounters the child and family outside their realm of authority and enters the child's home to a certain extent in the role of a guest (Yee and Andrews, 2006). While in some cases the home setting might empower the child in relation to the researcher, in others both researcher and child might have to deal with additional complex considerations in relation to the effect of parental authority in this setting (Valentine, 1999).

\section{Stakeholders' perspectives on ethical issues specific to children's research methodology and settings}

In the present study, stakeholders identified various research designs in relation to research with children, together with a variety of approaches (both qualitative and quantitative), a multiplicity of data collection methods and diverse research settings.

The importance of research design was recognised:

'There is an issue there around research design and appropriateness of research design ...

People think that they are going to use surveys with young kids, for example, or focus groups looking at issues ... That, I think, is challenging.' (AC)

The appropriateness of randomised control trials in particular was questioned in social science research settings:

'I have a concern from an ethical perspective on the obsession with randomised controlled trials research with children. I think that it raises huge ethical questions around denial of service. And just because people say it is right to use it [this approach] in medicine ... who says it is right in medicine? ... We're doing randomised controlled trials research on [names service], but we took the position that every child gets something [some service] at some point. OK, some might get additional [interventions] or it might be delayed, but there is no denial.' (CR)

The place of other approaches was considered:

'Now we have to do our work and we want to do high quality [research], but I don't think there's enough value on good ethnographic research with children. Even in terms of methodologies, we need to be a lot more respectful.' (CR) 
The need for research methodology to be child-focused and not adult-orientated was recognised: 'There is also the kind of research that may be appropriate for adults, like if they have some complex neurological form of assessment that maybe takes 2 or 3 hours to do. It may be very impractical for it to be done in children because children may not have the ability to sit still and go through a complex area for the same amount of time as an adult might. So they [researchers] need to open their research approach and methods just for children. And equally research questionnaires, if that is the instrument being used. It has to be carefully validated on children rather than simply taking one that has been used elsewhere, other than in Ireland, or apply ones that have been previously used for adults ... So, methodology has to be done in a way that is written for children or in a familycentred way. And that doesn't always happen, I'm afraid.' (HC)

The use of a range of approaches and perspectives for researching children's lives was perceived as challenging for the ethical review process, requiring a range of expertise:

'Perhaps you have people who come from engineering backgrounds who don't "get" qualitative research. They just don't get what's involved and they don't get that you can't tell them what questions you will be asking because that will emerge from the data.' (AC)

The nature of the process as challenging was not perceived as inherently negative:

'I think it is good to be challenged in all different [directions], quantitative and qualitative, whatever it is. But that they [committee members who assess a proposal] can understand your different perspectives.' (CR)

School as a setting was consistently raised:

'There really is no other way to contact children. There are very few other forums in which you have large numbers of children together. You just have to go through schools basically.' (CR)

The advantage of accessing children through schools was identified by a parent:

'School is the easiest way because you have your audience ... school is the way to go.' (P)

Parents also acknowledged an increased workload for the school if they were involved in the research. This was recognised as a potential challenge by researchers:

'I think the challenge is increasingly that schools, particularly in [region], are reluctant to take part in any research because there is so much research going on. Where once refusal was rare, refusal is now actually commonplace for all studies because schools are feeling increasingly under pressure.' (CR)

One researcher identified the role of schools from an ethics perspective as one of additional gatekeeper:

'On the whole, my experience has been that the school is yet another gatekeeper for the children, so it is adding another safeguard.' (CR)

However, another participant referred to researchers who had perceived the school as the only gatekeeper:

'Some researchers were not fully aware of ethical issues around accessing students ... For instance, it came up in terms of access to a school and using a classroom of children for research. Just because the school had some particular relationship to the researchers ... they hadn't realised that this did not give them carte blanche to go into the school and use the children, if you like, without getting the permission of their parents.' (AC)

The idea of gatekeeping was taken further by another participant:

'The other thing is, because they're sort of quite messy institutions and power-ridden institutions, they [researchers] also have to explore the notion of different gatekeepers ... if you're in a school where the Principal says "Yes, you can do the work", but the Principal is despised in the school, then ... [other staff will also gatekeep].' (CR) 
Specific issues identified in the school setting included children who did not have parental consent to participate in the research process:

'Another issue that has cropped up is what do you do with children in a classroom setting who don't have parental consent [to participate]? One of the big difficulties is then the exclusion of that child. So, are they going to sit down in the classroom, quite obviously sitting apart, and all the issues that that can raise for the child? Clearly you can't just let them out into the corridor. It's ensuring that people consider this issue and have an appropriate method of dealing with it.' (CM)

One researcher identified a perceived need for the development of some form of reciprocity in the relationship between the school and the researcher:

'I have been very conscious, as a researcher, of constantly going to schools and asking for help and support without ever really being in a position to offer anything in return. What I have been doing more recently is indicating in letters to schools that in the case of secondary schools I will be willing to offer career talks on psychology, should they be interested. In relation to primary schools, I have said that I will be willing to talk with parents on aspects of child development. So far, that hasn't been taken up by the parents.' (CR)

The role of teacher as researcher also required exploration and clarification:

'One of the big ones for us, given that a lot of people we deal with are teachers, is the power issue and for teachers, they are very straightforward. Very often they want to improve their own practice. The way to improve their own practice is to research the practice. The way to research the practice is to collect data from the young people in front. In school settings, if it's your teacher, how does that provide opportunities for the young person to opt out, to say "I don't want to participate in this process"? So, that whole power dynamic is one that ... the committee takes a fairly straightforward line on, which is that we think it is quite hard to justify doing research where the participants are your own students and where it is clear to them that you will know they have chosen to opt out in some way.' (AC)

One participant identified the need for the participation of primary school teachers in the review process for school-based research:

'What I found very useful on the ethics committee was having primary school teachers on it. I think that has been absolutely invaluable. Because their perspective really seems to stand out as quite different and quite acute as compared to all our views. I think they can bring an entirely different perspective, which is extremely useful.' (CM)

The contribution of teachers to the ethical review process in schools was also recognised by parents:

'The people who really know children and have a huge understanding are the teachers... Teachers are a huge resource that should be used.' (P)

However, a Chair of an academic REC went further, suggesting the level of expertise required warranted an independent dedicated research ethics review system:

'I' $m$ uncomfortable about approving studies using participants outside the university remit and ... I think the primary and secondary schools need to have representation when those decisions are being made within the university settings. You know, there is no ethical committee available for applicants to go and apply [to], but I would strongly support the development and setting up of ethics committees in primary and secondary schools. That doesn't have to be an ethics committee for each primary and secondary school ...' (AC) 


\section{Main concerns}

- Lack of methodological knowledge and inadequate application forms impair REC assessment of ethical issues in qualitative research.

- RECs' lack of familiarity with participatory methodologies may impede the realisation of children's research that uses these methodologies.

- Problems of both overestimation and underestimation of risk and vulnerability in social science research, especially in relation to children's research.

- General lack of clarity concerning good practice in school-based research, based on complexity of stakeholders' roles and relationships. 
6 ETHICAL ISSUES
IN CHILDREN'S
RESEARCH 


\section{Informed consent in research ethics}

\section{General concerns}

Informed consent is one of the major ethical issues in any kind of research with human participants and is acknowledged as essential by all major research ethics guidelines. All research proposals need to submit documentation regarding the issue of informed consent. It is frequently the area that receives the most detailed review attention, as evidenced by the international guidelines which frequently address informed consent requirements in considerable detail (HHS, 2005; NPSA, 2007).

Despite the general acknowledgement of the importance of participants' informed consent to research, it is by no means an uncontested field. Informed consent is probably the most researched area in contemporary research ethics, as seen in the extensive bibliography compiled by Sugarman et al (1999), detailing over 40 pages of empirical research on the subject. Discussions concerning informed consent most frequently address challenges in the practical implementation of informed consent procedures, especially regarding the problem of ensuring participants' understanding of information provided to them. While standardised informed consent forms that are signed by participants are still the most widely used method of obtaining informed consent, there have been concerns that in order to ensure participants' understanding, different additional methods need to be employed (Daugherty, 1999; Flory and Emanuel, 2004; Matsui et al, 2007). Especially for longer running protocols, continuing opportunities to explore and review informed consent issues need to be provided (Prentice et al, 2007). There is strong evidence that most informed consent forms used in research contexts are not easily understood and that recall of participants of information given to them is frequently patchy and faulty (Hochhauser, 1999; Lawson and Adamson, 1995; Meade and Howser, 1992; Paasche-Orlow et al, 2003; Stead et al, 2005; Tait et al, 2005). Current informed consent procedures are often focused on complex written documentation that does not take into account the needs of many participants, especially those who are less comfortable with this particular mode of delivery or lack the ability to process complex written information, as, for example, participants with lower educational levels, with relevant disabilities or from different cultural and linguistic backgrounds (Breese et al, 2007; Cox, 2002; Simon and Kodish, 2005).

Evidence has also been mounting that participants' perception of risk based on the information provided to them is often inaccurate (Biehl and Halpern-Felsher, 2001; Reynolds and Nelson, 2007). In clinical research, participants frequently have significant difficulties in understanding some research methodologies like randomised control trials (Cox, 2000; Daugherty et al, 1995; Joffe et al, 2001), although the extent of this misunderstanding might be unclear (Gordon et al, 2006). Participants generally seem to operate under the mistaken assumption (referred to as 'therapeutic misconception' in the literature) that participation in such trials will guarantee receipt of an effective therapeutic intervention (Appelbaum, 2002; Appelbaum et al, 2004; Bamberg and Budwig, 1992; Fisher, J.A., 2006; Jansen, 2006; Kimmelman, 2007; King et al, 2005; Miller and Brody, 2003; Miller and Joffe, 2006; Stead et al, 2005). One of the concerns in the literature has been what investigators contribute to this misunderstanding and whether there are effective means to avoid or correct the therapeutic misconception (Hochhauser, 2002; Jansen, 2006; Joffe et al, 2001; King, 2000). Underlying conflicts of interest on the researchers' side are frequent and might be one factor that may result in misleading participants about the precise nature of the research. Thus, efforts need to be made to make such conflicts of interest transparent and pay attention to them during the REC review process (Goldner, 2000; Larson et al, 2004; Resnik, 2000). It has also been pointed out that it is ethically short-sighted to focus too narrowly on the informationgiving parameters in the informed consent process, rather than take account of more general characteristics of the setting and social interaction (Corrigan, 2003). 


\section{Navigating informed consent and assent in children's research}

Research with children and adolescents faces the same problems as discussed above regarding informed consent, but these are compounded by further complications due to the following factors:

- the legal status of minors;

- the inclusion of additional parties, i.e. parents and/or guardians in the consent process;

- potentially, but not necessarily reduced psychological competence (as opposed to legal competence) for consent in minors;

- significant differences of knowledge and maturity between children of different ages and different study populations;

- significant inter-individual differences in maturity, even within the same age group or study population.

When adults decide to participate in research after receiving relevant information, their decision is usually considered binding, as long as there is no evidence of an unusual impairment of their mental competence. In contrast, a similar decision by a child or teenager does not have the same status of fully informed consent by a competent adult and is not sufficient for research to go ahead. Such a decision by a minor is considered to constitute assent, defined as 'a child's affirmative agreement to participate in research' (HHS, 2005, p. 1). A child's assent needs to be complemented by a decision of a 'legally recognised surrogate decision-maker' (Baylis et al, 1999), i.e. the child's parent and/or guardian. This means that a third party, or even a fourth, will need to be included in the consent process. These adult parties are placed in a position of decision-making authority based on the assumption that children or adolescents are not yet fully competent to make such decisions, especially not decisions that might involve some risk of harm. However, how exactly this assent process is realised in child research is not always clear from published literature and might indicate a need for further clarification for the research community (Range and Cotton, 1995a and 1995b; Roberts and Buckloh, 1995).

There is some confusion about the precise meaning of 'assent', especially regarding the question as to what extent a child's assent approximates an adult's consent, for example, in terms of the need for understanding and reasoned decision-making, as opposed to merely the acquiescence or opposition to a particular course of action (Denham and Nelson, 2002; Nelson and Reynolds, 2003; Wendler and Shah, 2003). As Joffe (2003) proposes, it might be appropriate to recast the question of assent in terms of what would constitute 'developmentally appropriate involvement in decisionmaking' and allow for a range of different forms of children's involvement, depending on their age, situation and maturity (Fisher, 2003b; Joffe, 2003; Nelson and Reynolds, 2003).

Bartholome (1996, quoted in Nelson and Reynolds, 2003) proposed that researchers need to address the following four elements regarding the assent process:

- help the child achieve a developmentally appropriate awareness of his or her condition;

- disclose the nature of the proposed intervention and the child's likely experience;

- assess the child's understanding and any (coercive) factors influencing the child;

- solicit the child's willingness to accept the proposed intervention.

One of the main concerns regarding assent is children's ability to understand information. A matter of dispute is at what point children have sufficient abilities to be actively involved in the informed consent process and what authority should be given to them depending on their level of competence (Ashcroft et al, 2003; Frader, 2003; Goodenough et al, 2003; Ladd, 2003; Weithorn and Campbell, 1982; Wendler and Shah, 2003). Views differ on what constitutes 'sufficient abilities', but it has increasingly become accepted that children should be actively involved in the informed consent process once they have some ability to conceptualise how research participation will affect them. However, there is also some evidence of the importance of emotional factors that might have a significant impact on cognitive processes (Dorn et al, 1995; Reynolds and Nelson, 2007 and 2008).

There is some dispute about the value of setting certain general age limits for involvement in assent procedures or for different levels of assent requirements, with those in favour highlighting that there are noticeable distinctions between different stages of child development (Halila and 
Lotjonen, 2003; McCabe, 1996; Toner and Schwartz, 2003; Wendler and Shah, 2003) and those against emphasising individual differences and the role of context (Ashcroft et al, 2003; Baylis and Downie, 2003; Fisher, 2003b; Goodenough et al, 2003; Halila and Lotjonen, 2003; Nelson and Reynolds, 2003). One common concern about fixed age limits is the worry that it might work as a disincentive to explore and employ methods of involvement for children under the age of the official cut-off point (Taylor, 2003). In these debates, authors on either side frequently refer to knowledge gained from empirical studies on children's developmental abilities and their performance in empirical studies on informed consent. One concern that has been raised in the literature is the question of how children's decisional capacity is assessed by researchers and whether these methods are ethically and psychologically appropriate (Hunter and Pierscionek, 2007; Kon and Klug, 2006).

While general concerns about the limitations in participants' understanding of information provided during the informed consent process are also encountered in relation to children (Ondrusek et al, 1998; Susman et al, 1992; Tait et al, 2003a), it is important to keep in mind that decision-making deficits encountered in children and adults alike should not lead to denying decision-making authority selectively to children, if it is likely that their parents will not be much better equipped to make these decisions (Fisher, 2003b; Halila and Lotjonen, 2003; Tait et al, 2003b). While there is evidence that children and adolescents have greater problems with expressions of probabilities and understanding of risk (Abramovitch et al, 1995), significant variability in understanding exists even among adults (Biehl and Halpern-Felsher, 2001). Generally, there is significant evidence that children much younger than the age of maturity can have a good understanding of complex medical treatment and make competent decisions on research participation. Evidence indicates that children over the age of 14 are virtually indistinguishable from adults in their decisionmaking capacities; that children in the age range 10-12 years show major similarities with older age groups; and even the decisions of younger children tend to be congruent with decisions of more mature decision-makers, even though they tend to be less able to provide arguments for their decisions (Bruzzese and Fisher, 2003; Burke et al, 2005; Cauffman and Steinberg, 1995; Schlam and Wood, 2000; Weithorn, 1983; Weithorn and Campbell, 1982). These findings are also compatible with findings that even comparatively young children who have extensive experience of participating in research have a highly sophisticated understanding of ethical concerns, if their views are elicited in relation to their concrete experience of research rather than through more abstract questioning (Goodenough et al, 2003).

One of the main concerns regarding the practice of information-sharing is how to tailor the information children receive to their cognitive abilities. Much attention has been paid to the adequate presentation of informed consent materials, in particular the information sheet and informed consent forms (Burke et al, 2005; Tait et al, 2005 and 2007). Some commentators, while not denying the need for suitable written information material, highlight the importance of the use of additional methods in order to be able to assess and improve children's understanding (Bray, 2007; Bruzzese and Fisher, 2003; Frader, 2003). In order to be able to develop age-appropriate information materials and to present them adequately to children, the researcher in charge of the information process needs to be familiar with the needs and characteristics of children of different age groups, and especially of the particular population to be included in the research (Broome et al, 2003; Holaday et al, 2007). One interesting proposal in this context that can help to address the frequent difficulties in preparing material that is accessible to children is to involve children themselves in the development of informed consent material (Ford et al, 2007).

How to combine the informed consent of parents or guardians with the assent of children is a frequently discussed issue. A general concern in this context is that not just investigators (Lederer and Grodin, 1994) but also parents might exert undue explicit or implicit pressure on their children to conform to their wishes, rather than allowing them to make up their own minds about research participation (Scherer, 1991). Adult authority has been shown to be a potentially significant factor in children's decision-making about participation in research and it can have a significant influence on decisions for uptake or continuation of research, regardless of the children's cognitive 
level of understanding (Abramovitch et al, 1995; Broome, 1999; Bruzzese and Fisher, 2003; Fisher, 2003b; McCabe, 1996; Scherer, 1991). The importance of social factors that might increase the perception of inequalities between researchers and participants has been pointed out (McDowell, 2001). There is evidence that behaviour of parents, children and researchers changes if the informed consent process takes place with all parties in one room, rather than parents and children engaging separately with the researcher (Broome et al, 2003). Accordingly, a phased informed consent process has been recommended, whereby children are given the opportunity to engage with the researcher on their own before making a decision (Geller et al, 2003). There is also some concern about the potentially unrealistic or inappropriate motives that parents might have to enrol their children in research (Fernandez, 2003; McGee, 2003).

However, while effects of undue pressure need to be detected and avoided as far as possible, it might not be appropriate to conceptualise parents and children as entirely separate agents in the decisionmaking process. Even for adults, decision-making usually has some relational element, insofar as 'significant others' are frequently consulted. Such processes are significantly more pronounced in childhood and adolescence, where parental consultation and guidance frequently play an integral role in children's decision-making and could possibly be used constructively in the informed consent process (Fisher, 2003b; Geller et al, 2003; Joffe, 2003; Broome and Richards, 2003).

One concern with regard to combination of parental consent and children's assent is what to do in the case of conflict or disagreement between minors and parents or guardians on their research participation. There is evidence from research with parents and adolescents that some disagreement between both parties is not uncommon (Brody et al, 2003a, 2003b and 2005a). As a general guideline, (1) preferences against research participation take precedence and (2) parents' decisions are given priority over children's decisions.

Usually, the parents' or guardians' decision against research participation is considered binding and voids any positive preference of the child to participate in research. Yet, a child's refusal to participate in research is also considered binding in most cases, even if parents have agreed to their child participating (Grover, 2003). This general rule applies to children and adolescents of all ages; however, there is some evidence that they may have little awareness of their right to refuse participation. Thus, sufficiently comprehensive assent procedures need to be in place to ensure children's awareness of their rights (Broome, 1999; Bruzzese and Fisher, 2003; Fisher, 2003b). However, when asked, children frequently seem to express the view that as participants they should have significant decision-making authority on participation (Broome et al, 2003; Geller et al, 2003). Ethical reasons to adhere to children's wishes are especially weighty if dissent is expressed by mature minors who have all the requisite psychological capacities, if not full legal authority, for making fully informed decisions (Baylis and Downie, 2003; Botkin, 2003; Toner and Schwartz, 2003). However, research ethics guidelines sometimes make allowance for cases where parents' wishes can override a child's refusal, as, for example, stated in US guidelines for consent to clinical research that has a likelihood of benefiting the child (HHS, 2005; Robinson, 2003) or in UK positions regarding the scope of Gillick competency (Kessel, 1993). There is also a lack of clarity over whether there might be potential exceptions to assent requirements in non-beneficial research (Murphy, 2003), but it has been argued that parental authority for proxy consent regardless of children's dissent would be unethical (Grover, 2003).

One concern that is raised frequently is the question of informed consent in research with mature teenagers. Due to their increasing abilities and maturity, members of this age group should be treated differently to younger children in terms of their ethical rights and responsibilities (Hofmann, 1980). In current practice, teenagers often already have significant freedoms to make decisions, for example, about their higher education or vocational training, religious orientation, sexual activity or decisions on medical interventions. Especially in relation to healthcare decision-making, adolescents have increasingly been given the right to consent or withdraw consent to certain types of treatment, including decisions with far-reaching implications (McCabe, 1996; McCabe et al, 1996; Weir and Peter, 1997). Some authors highlight the mismatch between the responsibilities and 
freedoms enjoyed by teenagers in certain areas of life and the frequent understanding that parental involvement in research decisions is required for all research participation (Sanci et al, 2004; Santelli et al, 1995). It has been argued that research ethics and legal guidelines are sometimes interpreted overly restrictively and that in the case of mature teenagers the age of consent for research participation can be lower than the age of majority (Santelli and Smith Rogers, 2002). Even where the legal situation would require parental consent, it has been argued that it would be ethically more appropriate to treat their decisions as fully informed consent rather than merely assent, and accordingly de-emphasise the role given to parents in the decision-making process (Botkin, 2003; Joffe, 2003). Especially given the data on adolescent cognitive abilities, authority to consent for low-risk research might be appropriate across the board beyond a certain age; even for higher risk research, consent based on an assessment of individual capacity might be appropriate (Santelli et al, 1995). Adolescents themselves seem to conceptualise their own role in the informed consent process as similarly significant, while parents more strongly emphasise their parental authority (Geller et al, 2003). However, there is evidence of a significant overlap between decisions by adolescents and parents (Brody et al, 2003b).

International research ethics guidelines differ significantly in the extent to which they assign decision-making authority to adolescents. In several jurisdictions, there are mature minor clauses that de facto allow adolescents full decision-making authority in healthcare matters. The English legal tradition allows for individual assessment of maturity based on the Gillick competence test (i.e. understanding of the nature and possible consequences of an intervention), which might allow independent decision-making for some children even under adolescent age. However, in the UK, it has also not yet been legally tested whether the transfer between decision-making authority for medical decision-making to the research area can be made (NPSA, 2007) and additional complications regarding the use of Gillick competency standards for health research have been highlighted (Hunter and Pierscionek, 2007; Piercy and Hargate, 2004).

While much of the Irish legal tradition is closely linked to UK law, due to special importance given to parental rights in the Irish Constitution it is unclear whether in the Irish context the UK Gillick test for mature minors can be applied at all; its applicability in the research context is particularly uncertain. In Australia, adolescents' ability to consent independently seems to have been introduced recently in research ethics guidelines (Sanci et al, 2004) and requires individual assessment (NHMRC, 2007). In other countries, age limits are in place: in Denmark, for example, minors over 15 years can be allowed to make independent decisions on research participation under certain circumstances (Government of Denmark, 2003), while in New Zealand adolescents over 16 years are allowed to consent without parental consent (NZHDEC, 2006).

Many RECs are unsure about the question of adolescent consent. There is evidence of great variability in decision-making on adolescent consent and a general tendency to favour cautious approaches to the matter (Mammel and Kaplan, 1995; Piercy and Hargate, 2004; Rogers et al, 1999; Wagener et al, 2004). There certainly seems to be a need to clarify the scope that RECs have in waiving parental informed consent requirements (Wagener et al, 2004) and there is an acknowledgement of the ethical weight of arguments in favour of full adolescent consent for at least certain categories of research (Levine, 1995; Mammel and Kaplan, 1995). Some proposals for ethical guidance for adolescent research have been made, highlighting the need for facilitating research to increase knowledge of adolescent health (Rogers et al, 1994; Santelli et al, 1995 and 2003).

One of the arguments that has been made against giving adolescents full decision-making authority is that while their cognitive capacities might be comparable to those of an adult, their decision-making capacities, especially with regard to dealing with risk in real life, are more questionable. Research has looked into the question of adolescent perception of risk and the difference between adolescent and parental perception of risk seems to be much less pronounced than expected (Quadrel et al, 1993); however, there is evidence that adolescents might be more willing to enrol in higher risk research protocols (Brody et al, Annett and Scherer, 2003a). Another interesting finding in this context is that both parents and adolescents might overestimate the degree of agreement between their views (ibid). 
The issue of independent adolescent consent becomes especially significant in research that involves sensitive subject areas and research on services that adolescents are using without their parents' knowledge. Such research might include, for example, research into teenage smoking (Diviak et al, 2004; Moolchan and Mermelstein, 2002), substance abuse (Rojas et al, 2008) or sexual health (Collogan and Fleischman, 2005; Flicker and Guta, 2008; Piercy and Hargate, 2004; Risjord and Greenberg, 2002). While teenagers may be entitled to use services without their parents' consent, they are sometimes considered to need parental consent to participate in research into the use of these services, a requirement which may make such research difficult to realise and might seem ethically arbitrary (Balen et al, 2006; Menasche, 1995). One focus of the literature in this area, particularly the field of adolescent sexual or mental health, has been the question whether and when waivers of parental consent might be justified and whether the use of such waivers could help to achieve suitable, unbiased samples (Crigger, 1996; Diviak et al, 2004; Fisher, 2003a; Flicker and Guta, 2008; Moolchan and Mermelstein, 2002; Piercy and Hargate, 2004; Risjord and Greenberg, 2002; Rojas et al, 2008; Sanci et al, 2004).

\section{Stakeholders' perspectives on informed consent}

In the present study, the main issue discussed by participants was that of the need and difficulties in relation to gaining consent from parents/guardians acting as proxies for children when undertaking ethical review of research with children. While other issues were raised, such as information requirements, the complexity of consent and research with children in relation to the role of parents/guardians in the process appears paramount. This complexity was acknowledged:

'The consent issue can be fairly complex, depending on the kind of topic, the age of the children.' (AC)

The levels of concern were also raised:

'There's the whole issue of proxy consent. The greater issue is for the proxy, the person giving consent for the child. There's massive amounts of concern about that.' (CR)

The concern was raised in regard to children perceived generally as a vulnerable group, but within this further levels of vulnerability were acknowledged:

'Often these children have difficult social circumstances as well, so they are a very vulnerable group, in which issues of consent are clearly very very paramount, but complex.' (AC)

The need for information about the research on which to base decisions in relation to participation was identified as key to the process of informed consent:

'I'd certainly say clear and detailed information is crucial.' (CM)

Other attributes required of the information included:

'Coherent, logical and sensible information about the research that is being done.' (HC)

The need for this information to be presented in a way that children, as well as adults, can understand was recognised. One Chair of a hospital REC suggested:

'I would argue that a well-written information leaflet that explains things to a 10-yearold would not need to be changed for an adult. The fact is that if you write it properly for young children, the legibility and understandability of those information leaflets will actually equally apply to adults. So what they should probably do is write the information leaflet for the 10-year-old and not change it for adults, rather than the other way round.' (HC)

Difficulties were identified in relation to gaining informed consent from children, even when information was provided:

'The people in the project explain it to them and they have the leaflet and all that, but I'd say they are just so used to just agreeing to things that there is a kind of an apathy there.' (CR)

Children, however, were not necessarily perceived as passive in the process:

'... children can defend themselves and they understand what it is about.' (CR) 
It was generally agreed that the age at which individuals can give consent is 18 years. Any person under this age requires parental/guardian consent. However, a certain lack of coherence nationally in the age at which young people are considered able to provide consent was identified:

'We have this situation at the moment where, for us to be safe, they must specify that they are aged 18 or over. And yet, they can have sex under 18, they can make their own decisions on medical procedures in hospitals at 16, they are coming here to college and choosing their own modules and their own degree programmes without needing written parental consent. And yet for a 17-year-old, we can't recruit them to fill out an anonymous survey on something that is totally non-contentious. We need to get that clarified.' (CR)

The nature of parental consent in research with children was discussed:

'At times you wonder is passive consent nearly better. That they read the letter and that you say to them, “Look, if you don't want your child to participate, please send back this form". But again that means, have they read the letter? I think if you are not actually handing it to them and they are there and can actually make that decision in front of you, I think it is very difficult to achieve informed consent. Because you don't really know what has happened.' (CM)

A number of further challenges related to proxy consent were identified by participants. A children's researcher identifying the different perspectives brought to the process by parents as opposed to their children:

'There was an example the other day. There was one young person: I'd spoken to his parents and they had one particular perspective and I spoke to him and his perspective was completely different. And he was so articulate and it was so informative, and I just thought that's why you need to do research with children.' (CM)

The majority of participants stated that in research with children, both the parent's permission and the child's was required. Parental permission alone was considered inadequate. This was especially the case for adolescents:

'It's all very well that you have a minor and have to have the parent's consent. But the position that we have had is that there has to be agreement by the person themselves [the minor]. You really cannot do anything with a 14-year-old unless they agree.' (HC)

'The principle we have here is that if you don't have both, you have nothing. So we look for parental permission and the child's permission, and really if either party is saying No, then we don't'. (CR)

This was recognised as challenging if one party did not provide consent:

'Where it gets very contentious is around adolescence because we come across adolescents who really want to [participate] and we think the adolescent is right, but we can't

because we can't get the parent. That actually happens more that way than the other way, I find. But it is very difficult.' (CR)

This raised other issues:

'Theoretically, if the child said No and the researcher respected that, then their consent is counting [i.e. valid]. So why go for proxy consent in the first place? It is an issue, in a sense, of consistency of approach.' (CR)

Consent was construed as an ongoing concept and participants pointed out that children should be, and are, able to withdraw at any time during the research process:

'They have the same withdrawal rights as any other participant.' (HC)

'I think it is important to remind them before you do something, like a workshop and during it, that they have the right to refuse to do it, even if they have signed it [consent form]. That they can leave at any stage.' (CM) 
In research through the school setting, in addition to parental and child consent, some participants identified the requirement for schools to consent:

'We require applicants to get formal permission from the school authorities and from the teacher and from parents, and in addition ... we will require the children to assent to the process as well. So yes, that is done ... on a formal basis.' (AC)

Some discussion was generated by consideration of specific situations when parental consent may be waived, as, for example, in specific situations in the school setting, according to one Chair of an academic REC:

'I imagine if, for example, only teachers were involved in the process. If you were asking teachers to report on their teaching practice. In such cases, if there were no recordings we probably would feel that it wasn't necessary because children are not directly involved, although they are indirectly involved.' (AC)

Another area given as an example was public health research with neonates:

'Some of the work is trickier, in terms of neonates. Again, you come back to the other issues of doing surveys, chart reviews, they can't get consent ... It's public health research and I guess I led the committee in saying, the idea of public health research seeking individual consent, you can't do it [especially in relation to] mortality, etc.' (HC)

However, waiving the need for parental consent was considered to be the exception:

'It came up again in research we are doing for the [name of organisation], where we were trying to involve children, young people who were in an association for lesbian [and] gay young people ... and we decided that we could only invite people who already had "come out" to their parents ... So in that case, the issue of inclusiveness had to give way to the other issue of non-maleficence ... Well, I know that there is reverse [i.e. different practices in research] out there, in Denmark for example, where they were asking children about, I forget now what it was, but they made the firm decision they would not ask the parents because if they did, they wouldn't get responses from the children and be told the truth. I think it was a big survey, it wasn't a personal one-to-one thing either, you know.' (AC)

The issue of the collection of data in children's research for one purpose with the subsequent use for another - in essence, secondary analysis - was raised as an issue in relation to consent:

'In terms of things like photographs, representations of children ... for instance, Travellers tend to be used quite extensively and I think you know there are ethical questions around that now at school. I mean, that would be something I would have seen and flagged in other cases - that any group is not to be used photographically again without full consent and awareness of what the pictures are for.' (AC)

The challenges raised by informed consent in research with children resulted in one Chair suggesting:

'It would be ... nice ... if there was some kind of central regulation on consent and on what was good practice there. And especially parental and child consent, because obviously the younger the children get, the more difficult it will be to get proper consent.' (AC)

\section{Parents' perspectives}

The criteria that would influence the decision by parents to give their informed consent or otherwise for their child to participate in research were explored. The parents were all very definite that they need clear and transparent explanations of what the research is about, who is doing it and what the data will be used for:

'I would allow my daughter to be involved if I was very clear what she was taking part in and there were no grey areas ... if I could see every step of the way.' (P)

'What is the research being used for ... what is the purpose of it and to have a good and clear explanation of it would be critical.' (P) 
There was clear resistance to allowing children to become involved in research that is commercial or about marketing:

'Specific thing is that it is not a marketing-type thing, that that would be clearly stated. That would be the key thing to know, what it's used for.' (P)

'If it's going to a pharmaceutical company, then I wouldn't be that interested.' (P)

The relevance of the issue under examination to the particular child was also seen as important:

'Presumably, if the issue was of relevance to their child would be the most logical reason for allowing their child to be involved.' (P)

Fitting with parents' belief systems was also important:

'Depends on whose running it and what it was for and whether it was values that we held to.' (P)

The participants stressed that it is they as parents who need to make the final decision about their child's involvement in any research, rather than the school's decision:

'I don't think that, unless they have parents' approval, schools should decide.' (P)

However, parents did discuss the role of the school in relation to the consent process:

'You would kind of expect them [schools] to vet the research, to find out what's involved, not just be some kind of a broker.' (P)

'School makes sure everything is in proper order for things done in school.' (P)

Consent forms and information being sent home by the schools were seen as probably the best way of gaining parental consent:

'Quite reasonable ... to go through the school with the note and put on a phone number, so the parents can ring you personally, be very open with a name and a number.' (P)

Parents were also aware of the limitations:

'An awful lot of notes get lost or get stuck in the bin.' (P)

Interestingly, there was very limited reference by parents to issues pertaining to gaining children's consent to participate in research independently of the schools or the parents. Any discussion was specifically related to asking difficult questions, rather than participating generally in research.

Reference was made to information being provided directly to children:

'I suppose if it's explained properly to them [parents] beforehand, it might clear up misunderstandings or misconceptions of what information they [children] are being asked for.' (P)

Reference was also made to ongoing consent.

'Yes, if they have the chance to say "I don't wish to answer that". You can't force them to answer, but they should actually know that they can say No. That would have to be established in the ground rules.' ( $P$ )

\section{Children's perspectives}

In the participatory workshops with children in a school setting, the participants were clear that they have an active role in the consent process, apparently accepting that others - namely, parents and school Principals - would be involved. The reason for parental involvement was recognised as 'It's a way of parents protecting them' (C).

Participants identified that a child's consent should precede parental consent. As one participant stated, 'Should ask Principal, then us, then parents' (C). 
This was reiterated by another participant, who considered that children's consent should be sought and recorded at the top of any consent form, followed by parents. Participants pointed out that children actually act as gatekeepers in relation to the forms seeking consent that they choose to take home and present to their parents. In this way, participants stated that they influence the consent process. Participants were asked about their perception of choice, following written parental consent: 'You have a say in this as well' (C) and 'You can if you want, you don't have to' (C).

The role of peer pressure was acknowledged if potential research participants were asked to consent in a group situation. Refusing to participate was identified as potentially difficult: 'Walking out might be a bit embarrassing' $(\mathrm{C})$.

The role of information to inform choice to participate in research was recognised as a prerequisite to consent. Participants suggested that multiple approaches over time to information provision were necessary in order to ensure potential participants were informed. Suggestions included researchers visiting potential participants prior to data collection and explaining the study, as well as 'Talk to them, one to one' (C) and 'Write it down' (C).

The young people were almost unanimous in their belief that researchers need to inform the participants about the nature of the research and what was involved before asking for their consent. This will give young people the opportunity 'to be prepared' $(C)$ and 'better to expect the unexpected' (C). One participant stated that researchers should 'name out what you will be talking about' (C).

In one school, in addition to the researchers' information, the Principal had also explained the research process to the children and participants considered this to be beneficial. Another important consideration was to supply information on alternative activities if people did not wish to participate in the research.

Participants considered inducement to consent to take part in research would be advantageous in encouraging participation and between them they provided a list of incentives, which included 'Two-day summer camp' (C); 'Give them sweets' (C); 'Give them money' (C); 'No more homework' (C); 'Improve the football pitch' (C); 'Buy us soccer balls' (C); and 'Clothes and makeup' (C). No limitations were expressed on this approach to recruiting research participants.

\section{Confidentiality in research}

Participant confidentiality is a significant concern in research ethics and is given a prominent place in most research ethics guidelines and REC applications. Usually, confidentiality issues are also governed by certain legal data protection requirements and ethical research requires familiarity with these legal requirements (SSHWC, 2007a). The main concern about confidentiality in research relates to information that has been provided by the participant to the researcher or to data that can be traced to the participant. The responsibility of the researcher is to make sure that data are not available to unauthorised persons. This includes several different issues:

- safe data storage (physical and electronic);

- removing identifying information from datasets, for example, by encryption, anonymisation or modification of identifying information;

- receiving approval from participant for any disclosure of information to other parties.

First of all, data that have the potential of being traceable to a particular participant should be kept physically out of reach of unauthorised persons. Data that contain any identifying information (e.g. non-anonymous questionnaires, recordings, transcripts, photographs and any document that links anonymised data with identifying information) should be kept in a locked filing cabinet that can only be accessed by authorised persons. For especially sensitive data, it is sometimes advised to consider encryption of files. Similarly, for data stored in electronic format, the computer on which the files are kept needs to be password-protected and there also needs to be a policy in 
place regarding what constitutes legitimate forms of transfer of data (e.g. whether it is considered appropriate to move data on USB keys, share files via e-mail, send data in certain formats to other sites or use a professional transcription service) (Easter et al, 2004).

Ensuring that anonymisation is indeed protective of participants' confidentiality is another significant concern. Anonymisation may turn out to be difficult to achieve in qualitative research, especially when recruiting from a small population or when using case histories. Often, identifying information might be exactly the kind of information that will yield important insight into the research question. Researchers are faced with the challenge to anonymise data by modifying some parameters, including distracting information, without affecting the validity of the data (SSHWC, 2007a; Wiles et al, 2006). At the same time, there is also a danger that participants might be alienated by some of the methods used to achieve anonymity (Grinyer, 2002).

One challenge that can be faced in the research process is research that involves methods like focus groups, where participants interact and share information with other participants. While it should be expected that the researchers are aware of their obligation to conform to standards of confidentiality, participants may be less clear on what their obligations are. On the one hand, it is the researcher's responsibility to make sure that participants are made aware of their obligation to keep information they gained from other participants confidential. It might also be advisable to emphasise to participants that they should be aware of the possibility of 'leaks' and that it might be advisable for them to be careful about how much information they want to reveal within the group. A somewhat related challenge can be encountered even in individual interviews, in that participants might provide information on other people or parties who themselves have not consented to being involved in the research (Hadjistavropoulos and Smythe, 2001).

Another major concern regarding confidentiality is the participant's right to determine - and veto - who is allowed to receive personal information beyond the research context. Sometimes, information that is provided to the researcher in the research process may be of interest to other parties. The researcher is generally obliged to keep this information confidential, unless a legal obligation of disclosure exists, for example, in the case of disclosure of certain criminal activities, child abuse or - in some legislations - disclosure of the participant's intention to severely harm another person or themselves. Whether such information is likely to arise can often be determined in advance, depending on the research population or the topic of research; however, it is possible researchers might encounter such information unexpectedly. While the general consensus is that the possibility of disclosure needs to be communicated to the participant before conducting the research, there is evidence from other areas of practice that communication about limits of confidentiality has a significant influence on what interviewees might disclose (Woods and McNamara, 1980).

However, one challenge in this area is the increasing demand to create data-sharing and pooling opportunities both in the clinical and social sciences for scientific benefit. These practices have been strongly endorsed by some researchers and funding bodies (Cooper, 2007; De Wolf et al, 2005 and 2006b; Sieber, 1991). Whether and if so under what circumstances these practices can be compatible with participants' right to determine the use of their data has not yet been satisfactorily solved. This is also related to another concern that is often brought up in relation to confidentiality and informed consent requirements - namely, the conflict between patients' rights and the effect such requirements can have on the feasibility of public health and epidemiological research and other research that relies on the review of patient charts and databases without requiring additional research interventions (Dane and Parish, 2006; Hewison and Haines, 2006; McCarthy et al, 1999; Meslin, 2006; Taube and Burkhardt, 1997; Tu et al, 2004) or the use of tissues in tissue banks (Wolf and Lo, 2004). Canadian regulations require REC review for most such research, but acknowledge the social importance of the realisation of this type of research and its potential disruption by strict interpretations of the IC rule (CIHR et al, 1998/2005). In the USA, new regulations in relation to the Health Insurance Portability and Accountability Act (HIPAA) have had a significant effect on the question of access to patient data and have been the subject of controversial discussion (Armstrong et al, 2005; Breese et al, 2007; De Wolf et al, 2006a; Meslin, 2006; Ness, 2007; Sobel, 2007). 


\section{Confidentiality in children's research}

In the case of research with children, all of the above concerns should be taken into account, but there are also some challenges specific to children's research. Firstly, due to children's legal status and their potentially limited psychological competence, the role of parents or guardians needs to be taken into account. They may expect to be informed of any information arising from the research that might be relevant to the child's care or well-being. Especially in relation to clinical or psychological research, there is a reasonable expectation that parents, who have decision-making authority on healthcare and educational matters, will be informed of any significant diagnostic information arising from research activities. In explaining confidentiality, any such limitations to confidentiality will need to be discussed with parents and children.

One area of concern that is particularly prominent in research with children is child protection. In most jurisdictions, including Ireland, mandatory reporting laws and relevant child protection guidelines are in place (OMCYA, 1999/2009). These require researchers to report to the authorities if information they gain during their research indicates that a child might be abused, even though this could mean a breach of confidentiality, a potentially serious ethical concern. It is considered good practice to communicate these limitations to research confidentiality to both parents and children during the informed consent process (Neill, 2005). One concern in relation to this requirement is that it might be detrimental to certain types of research that depend on the establishment of a relationship of trust and openness, as Bostock (2002) reported for child protection, Fontes (2004) for violence against women and Sieber (1994) for child abuse and neglect. Concerns about confidentiality, or more generally about being faced with difficult questions by researchers, might lead to significant difficulties in recruiting participants for studies in sensitive areas (Walsh, 2005).

Data from social work practice indicate that practitioners frequently avoid broaching certain topics in order to preserve a good quality of relationship (McLaren, 2007); it can be expected that researchers might behave similarly (Sieber, 1994). While some researchers favour an approach that will not go against the child's wishes and focuses on supporting the child in reaching decisions about raising an issue in a way they are comfortable with (Thomas and O'Kane, 1998), generally mandatory reporting requirements are taken to be non-negotiable. However, these situations are characterised by significant complexities and require further ethical attention, especially regarding social and emotional factors that might potentially lead to over-reporting or under-reporting (Fisher, 1994; Liss, 1994; Scarr, 1994; Scott-Jones, 1994; Sieber, 1994; Williamson et al, 2005).

In addition to specific child protection concerns, other issues related to confidentiality limitations (e.g. the risk of suicide) can also arise in minors' healthcare and health research. There is evidence that concerns about limitations of confidentiality may have a significant effect on the disclosure of relevant mental health information provided by participants (English and Ford, 2007; Lehrer et al, 2007; Lothen-Kline et al, 2003).

As already indicated in relation to informed consent, the situation of teenagers is particularly complex. With increasing age, they are given increasing rights of self-determination in many areas of their lives and this should have implications for the issue of their rights of confidentiality in healthcare and health research (Ford et al, 2004; Hofmann, 1980; Jackson and Hafemeister, 2001). With regard to some aspects of healthcare and services, such as sexual health services (Jackson and Hafemeister, 2001), adolescents are often entitled to confidential access to services. However, there is evidence that parents and teenagers might not be aware of relevant legislation and practices regarding confidentiality of teenagers' service use (Lyren et al, 2004 and 2006; Resnick et al, 2003).

In relation to health services, there is evidence that teenagers might be less aware of existing protections than of limitations to confidentiality, and might therefore underestimate the degree to which they have a right to confidentiality (Ford et al, 2001). In relation to research, it is not always clear whether the rights that participants have regarding access to services also apply to research conducted in the same area (Brooks-Gunn and Rotheram-Borus, 1994). If the informed 
consent process for research requires involvement of both parents and teenagers, it will need to be discussed with both parties what type of information might be shared with parents or guardians and what will remain strictly confidential. There is evidence that in research concerning sensitive areas, expectations about disclosure from both parties might differ significantly from existing policies and the researcher needs to clarify these in advance (Lyren et al, 2004 and 2006; Pasternak et al, 2005). In research on services, possible limitations of confidentiality need to be considered carefully, including the issue of the danger of discouraging teenagers from accessing important health services (Jackson and Hafemeister, 2001) and the question of how to deal with the fact that research may accidentally uncover, or even directly address, teenagers' behaviour that is illegal (Moolchan and Mermelstein, 2002).

\section{Stakeholders' perspectives on confidentiality}

Generally, there was recognition of the limitations of confidentiality in research particularly with children, both implicit and explicit. Implicit limitations identified included:

'Sometimes researchers are inclined to be a little blithe in the assurances they can give about confidentiality. They don't realise that if you work with a particular cohort of children in a particular kind of school that the actual children may be recognisable because the numbers are so small. That is an issue that we have addressed, but there are continually issues such as that.' (AC)

There were also implicit limitations on children as participants:

'Children themselves don't understand confidentiality. If I say to research participants, "This is confidential", how do you explain confidentiality, particularly within a group? And, in effect, you can't say that because they have to be able to go home and tell their parents.' (CR)

However, explicit limitations to confidentiality were also identified with reference to a duty to report, generally in relation to 'danger':

'Our general guidelines are that confidentiality is limited. We always have a consent about if any information is disclosed ... [information that] indicates that the person might be at risk of danger. Then they have to know that you are going to take it further.' (AC)

This point was highlighted specifically in relation to child protection:

'But you have to assume that there is a chance that a child will say something that makes you [think] there is an issue of child protection. And you have to prepare for that. So when we go into focus groups or interviews with children, we would always inform parents and children in advance that the interview is confidential to the research unless a child says something that makes us think they are at risk. And if this happens, we will have to report it.' (CR)

Confidentiality was also discussed in relation to illegal activity:

'We do have university guidelines ... on confidentiality. If someone is told in confidence or in, say, a questionnaire that a student is involved with alcohol, drugs or something illegal, then we have a duty of reporting that.' (AC)

Confidentiality in relation to visual images was identified as a particular issue:

'Privacy concerns us quite a lot in videos and ... the video issue is complex ... We've designed a set of regulations in terms of if videos are taken, how they are stored, how they're handled ... the security of them, etc. ... We've created our own handling regulations ... the tape must be stored by the academic, not by the student ... it's been kept for a certain amount of time and it has to be destroyed at a certain stage ... that sort of thing.' (AC) 


\section{Parents' perspectives}

Parents made limited reference to confidentiality, but did refer to the need for anonymity:

'Anonymity would be high on my list, in that no person reading it would be able to identify a parent or a child.' (P)

Parents also stated that they would like to know that the data are being securely stored and will be destroyed once analysed and used:

‘[You] read about laptops being stolen ... breeding distrust in data and things being stored. That would worry me now ... I thought it was good that the data would be destroyed after a certain amount of time ... that it's not being passed on.' (P)

One parent raised the issue that if the research results found something 'untoward', then the parents would like to know this and that this information could be fed back to the parents via the school:

'Results would be important to know and to point out particular pieces of data that the researchers have found that may be worrying for particular parents. So point it out to the Principals, who can tell the parents.' (P)

There was general agreement that feedback is important and by being able to see the results of the research, parents are reassured as to who has had access to the data given by their children:

'Know where information or where the name has been passed on [to], to see the traceability.' (P)

\section{Children's perspectives}

Participants expressed a range of perspectives on issues of confidentiality when presented with the dilemma of a child disclosing disturbing information to a researcher, such as abuse or neglect. In this situation, a few participants considered confidentiality to be sacrosanct, so that researchers should never divulge information: 'Because it's confidential' (C) and 'It's a contract' (C).

The participants with this perspective held their view throughout the discussion. Some participants, however, were unsure about what should be done, one stating: 'Sometimes you mightn't want to get involved and move in [on] someone else's business' (C).

However, the majority of participants expressed the opinion that such disclosed information should be acted upon, even if it meant breaking confidentiality. Justification for this was given: 'He would be very depressed and it might end up leading to suicide' (C) and 'But maybe they need help' (C).

Although there was no consensus on who should be informed, suggestions included parents, the school Principal or the class teacher. It was also thought that the researcher should contact the child in question and explore the issue further. Reasons for and against these various approaches were also proffered. Informing the teacher, who in turn would discuss the issue with the class was suggested: 'Tell the teacher to talk to them, but not the exact person' (C). The role of the Principal was identified as the person who could actually do something positive about the situation: 'The Principal could stop it' (C).

There was less agreement about letting the parents know and some interesting arguments took place. There were those in favour of involving parents: 'Then they should ask for help from their parents' (C). The result of this might be that 'The parents might talk to their children' (C).

Those against the idea of informing parents considered that it would be 'making too much of a deal about it' (C). The result of this, as one participant stated, might be 'that parents might get worried' (C). Although in response to this, another participant stated, 'He is not going to get killed' (C). 
The role of the researcher in this situation was also discussed and some participants considered that the researcher themselves should intervene, although one participant pointed out that 'If you [the researcher] ring their [the child's] home, they would be wondering ... upset ... and then the parent tells the teacher ... catastrophe' (C).

It was suggested that the researcher might be able to gain access to the child by telephone without the parents knowing and that this would be a better situation. The justification for this was 'If he wanted help, he would have told his parents' (C). The problem with breaching confidentiality was stated to be 'Then everyone will know about them' (C).

\section{Risk and benefit}

\section{Generic concerns regarding risk, burden and benefit}

One of the most fundamental concerns in research ethics is the protection of participants. The primary goal of existing research ethics structures and practices is to ensure that participants will not be exposed to undue risks and, where possible, receive some benefit from their research participation. However, as long as sufficient general protections are in place and participants have been adequately informed about the research and its risks in the informed consent process, participants are then considered competent to decide for themselves whether they want to face some risk. Additional protections are usually put in place for research with more vulnerable groups, to avoid harm or exploitation, even if such participants could be considered competent (0wen, 2001).

Regarding the assessment of risk by participants, there is one important caveat: lay persons find it generally extremely difficult to conceptualise risk. As has been repeatedly shown in studies on risk perception, both in research contexts and outside them, people without a significant degree of mathematical or scientific understanding make many mistakes in conceptualising risk, mistakes that seem to work towards both underestimating and overestimating risk under some circumstances. First of all, there are significant problems with translating risk that is expressed in percentages into a concrete understanding of the extent of the risk. In addition, there is the wellknown problem of the framing of risk. Different descriptions of risk, even though mathematically equivalent, can lead to significant differences in whether people take these risks to be acceptable or not. For example, while a risk that is described as 'equivalent to the risk encountered in using a car' seems acceptable to most, a risk that is described as a certain, minimal 'likelihood of death' is more frequently considered to be unacceptable, despite there being no difference in the extent of risk faced in either (Wendler et al, 2005). There have been some attempts to make sure that informed consent processes and materials correct for these challenges in presenting risks, but accurate risk assessment still presents a significant problem for participants. Accordingly, this might justify a more conservative and paternalistic stance in relation to the extent of risk that might be allowed in research.

Clinical trials research is one of the areas where the potential for harm is particularly significant. When testing substances or interventions in human beings for the first time, research participants are exposed to risks that are probably harder to predict than in other forms of research. Accordingly, the most stringent research ethics regulations can be found in this area, requiring not just careful ethical planning of the research, but also ongoing risk monitoring. Internationally, when the research ethics system has been actively reviewed and reformed, it has been mostly specifically in relation to medical research, and especially clinical trials research. For example, on a European level, the Clinical Trials Directive mandates the EU Member States to fulfil comparable standards and practices in the review, monitoring and safety reporting of clinical trials. No such obligation exists with regard to other types of research. However, while participants in clinical trials are largely motivated by the hope of therapeutic benefits, they seem to be adequately aware of risks involved in clinical trials on new therapeutic agents (Daugherty et al, 1995). 
One concern in relation to risk is the assessment and quantification of risks. In addition to the problems of uncertainty and prediction, there are also some more fundamental concerns. It is notoriously difficult to assess psychological risks and, as pointed out by researchers and REC members, there is evidence not only of significant conservatism and risk adversity in relation to identified psychological risks (Corbin and Morse, 2003), but also of a lack of awareness of certain psychological risks or harms (Hadjistavropoulos and Smythe, 2001). There is also some evidence that risk may be determined merely by reference to the subject area that is going to be researched (Wagener et al, 2004): it seems that the risk of participants being upset by research in sensitive areas is often overestimated by REC members and can be perceived as much less problematic by research participants (Cromer et al, 2006; Scott et al, 2002).

In relation to the issue of harm to the participant, it is important to consider two aspects - the risk of harm as a consequence of research interventions and the burden of research participation itself. Even though this burden is usually not of a spectacular nature, it needs to be considered. Participants are frequently asked to spend significant amounts of time with research activities, especially if the research requires them to attend several meetings or follow-up sessions. They may also be required to complete comparatively tedious tasks, such as filling in long questionnaires or engaging in highly repetitive activities. It is therefore important for researchers to make the burden on participants commensurate with the potential significance of gain in knowledge and to design their research in a way that overall minimises the burden on participants.

Arranging for post-completion contact is one way of bringing potential negative effects of the research activities to the attention of the researcher. Post-completion contact can include:

- debriefing after completing the research;

- follow-up with participants or relevant contact persons;

- feedback on completed research.

Debriefing allows research participants to feed back their experience or any questions or concerns they might have to the researcher, usually immediately after the completion of the research activities. It is also an opportunity for the researcher to clarify certain aspects of the research that could not be addressed beforehand. Especially in the case of research that involves an element of deception, debriefing is essential from an ethical point of view, not only to provide the participant with a good understanding of the research, but also to allow researcher and participant to engage with each other regarding the deceptive nature of the research. Follow-up involves direct contact with the participant, a surrogate or another relevant contact person in order to enquire about the effect of the research participation or in order to share information arising from the research that had been agreed on during the informed consent process (e.g. concerning relevant diagnostic information). The possibility and likely circumstances of follow-up contacts should be agreed in advance with the participant, so that the best mode of conveying relevant information can be determined and contact by the researcher does not come as a surprise.

Feedback on completed research is much less frequently mentioned in the literature and there is no clear standard regarding feedback. In general, it is considered desirable to make information on research results available to participants. However, there are no generally agreed best practice standards for feedback to participants. Some researchers offer to contact those participants who explicitly express an interest in receiving results. Researchers may offer a copy of the relevant academic publications arising from the research or they may prepare a 'user-friendly' version, written in such a way as to be accessible to the general public or the particular research population. In some research projects, especially if participants have been involved extensively, feedback might be given at a special event that is dedicated to the presentation of the research results.

In addition to avoiding risk and burdens, researchers also face the demand to allow participants, where possible, to benefit from their participation in the research. This demand has been especially strongly emphasised in guidelines on research with vulnerable populations, who can usually only be recruited to research that has a potential to benefit themselves or, in some circumstances, people who are in a similar situation to their own condition or illness. 
Among the more frequently mentioned benefits of research participation are the following:

- access to otherwise not available services, diagnosis or treatment (Edwards, 2006);

- gaining novel experience and information through research participation and feedback;

- altruistic satisfaction in being part of a process that might contribute to improvement in therapies and services or to an increase in knowledge.

These potential benefits are considered significant by many researchers. There has been some debate on the problem of under-representation of certain populations in clinical trials, such as women, minorities or socially disadvantaged groups. Such under-representation is not just a concern due to a potential lack of validity of research that is based on biased samples, but in these discussions research participation is presented as a good that should be distributed equally to members of different populations.

The benefits arising from research participation are frequently emphasised by researchers in their recruitment activities and material. However, there is a danger of overstating the extent of these potential benefits to participants. Especially in the clinical context, it is important to keep in mind the tendency of participants to presume a direct therapeutic intention on the part of the researcher. Emphasising these aspects too strongly might distract participants unduly from the non-beneficial aspects of the research.

One concern that has been raised in this context is the extent to which remuneration for research participation should be considered a benefit for participants. Research ethics guidelines tend to be ambiguous on the matter. On the one hand, a primary concern is that the amount of available remuneration should not be a primary incentive for potential participants to participate in research. Offering substantial remuneration is generally perceived as a form of potential coercion for participants, which might lead them to disregard risk, especially if they are in financial need (NZHDEC, 2006). Underlying this is the paternalistic assumption that participants may need to be protected from themselves and are not to be trusted to make appropriate assessments on how to offset the receipt of remuneration with the risks that they might face.

On the other hand, it has been pointed out that participants' purely altruistic involvement in research should not just be taken for granted. There is some evidence that for some types of research the use of participant payment might improve access to participants and, accordingly, the quality of sampling (Croft et al, 2007; Thompson, 1996). Even though altruistic motivation can be assumed in many cases, there is disagreement about whether it should be an ethical requirement for research participation in otherwise non-beneficial research. Some authors argue that it would only be fair to give some acknowledgment to participants' effort and input; this has also been confirmed by research participants themselves (Russell et al, 2000). While some prefer a token of appreciation without significant monetary value (e.g. cinema vouchers or small amounts of money), other suggestions have been that participants should receive compensation for the costs they incur through participating in the research. This might mean either transportation costs and potentially other expenses, or a compensation that is relative to the actual time spent in participation (e.g. paying participants the minimum wage for time spent on research). Another option that is sometimes adopted is the use of lotteries, whereby participants enter a draw for a more substantial prize (Brown et al, 2006). There is evidence that there is substantial disagreement among each of the stakeholder groups - REC members, researchers and research participants - regarding what level of payment would be considered ethically appropriate (Ripley et al, 2006; Russell et al, 2000).

\section{Risk and benefit in research with children}

In research with children, the protections are significantly stricter than in research with adults, based on the assumption that children are more vulnerable and open to risk than adults. As indicated above, in some circumstances adults can decide to participate in risky research, while in the case of children's research the situation is much more restrictive. Not only are children not entitled to make such decisions on their own, due to concerns about their cognitive competence and decision-making authority, but their parents or guardians are also faced with restrictions about what research they can agree to on behalf of their children. 
One of the main reasons for a comparatively restrictive attitude to risk in research with children is the issue of vulnerability. Children and adolescents are traditionally conceptualised as a particularly vulnerable group in research ethics due to their lack of maturity and limited decision-making competence, but also due to their limited social and legal power vis-à-vis the researcher and their parents (Morrow, 2005; Thompson, 1990). While in many respects, children's vulnerability decreases with increasing age and maturity, some psychological vulnerabilities increase with the development of certain cognitive capacities and therefore can become more pronounced with increasing age, which makes a good awareness of child development an important precondition of responsible research with children (Thompson, 1990). However, the conceptualisation of children as a vulnerable population who need to be protected from dangers has received some criticism by those who argue that this perception of children, primarily as potentially helpless victims, might have the effect of disenfranchising them by preventing their participation in research and having their voice heard unmediated by parents or other adults (Mishna et al, 2004; Morrow, 2005). The views of children and parents can differ significantly on the role of risk and risk management in their lives (Hood et al, 1996; Kelley et al, 1997). Especially in recent years, there has been a significant increase in children's research that has attempted to envisage research as a constructive exercise with the potential of gauging children's perspectives and contributing to their further empowerment (Alderson and Morrow, 2004; Farrell, 2005; MacNaughton and Smith, 2005; Morrow, 2005). Nevertheless, a competence and empowerment perspective should not distract from the need to use special care in conducting research with children.

The concern about vulnerability becomes particularly significant in the case of research with children and adolescents whose vulnerability might be increased because of factors like serious illness, disability, mental health issues, abuse, sexual orientation or personal and social circumstances (Bogolub and Thomas, 2005; Cocks, 2006; Cuskelly, 2005; Fontes, 2004; Helgeland, 2005; Hoop et al, 2008; Kennedy, 2006; Scott et al, 2006; Solyom and Moreno, 2005; Thomas and O'Kane, 1998; Thompson, 1990; Valentine et al, 2001; Yan and Munir, 2004). Historically, many cases of children's research have been conducted with particularly vulnerable groups of children and concerns about their exploitation are understandable in this context (Lederer and Grodin, 1994; Lederer, 2003). On the other hand, there is a particular imperative to conduct research on issues that are relevant to these children's lives because they have special needs and therefore require well-founded, effective services. Some authors are concerned that sometimes the assumption of participants' vulnerability might lead to misguided attempts at protection, resulting in their exclusion and disenfranchisement as research participants (Cuskelly, 2005; Helgeland, 2005). With regard to different needs and abilities of different populations, another concern that is brought up frequently in the literature is the level of maturity and experience of children who are suffering from chronic or life-threatening illnesses. While they might be considered particularly vulnerable, they can also be considered to have had experiences and developed maturity and abilities of understanding that are unusually advanced for their age. These factors might need to be taken into account in research planning and the implementation of particular approaches to their protection (Fernandez, 2003; Sharp and Quigley, 2003).

Most international guidelines agree that children require particular protection in research and, accordingly, set fairly strict demands on the issue of risk. The US guidelines on risk in children's research spell out the requirements in some detail (HHS, 2005). Regarding risk, they distinguish between the following categories of approvable research:

- Research with minimal risk, defined as 'the probability and magnitude of harm or discomfort anticipated in the research are not greater in and of themselves than those ordinarily encountered in daily life or during the performance of routine physical or psychological examinations or tests' (45 CFR 46.102(i)).

- Research involving greater than minimal risk, but presenting the prospect of direct benefit to the individual subjects, and where the risk-benefit ratio is likely to be equivalent or better to existing interventions. 
- Research involving a minor increase over minimal risk, presenting experiences that are similar to those encountered in their particular current situation (e.g. medical, social, education), no prospect of direct benefit to individual subjects, but likely to yield generalisable knowledge about the subject's disorder or condition (often referred to as 'subject condition requirement').

- Research not otherwise covered by the above criteria which presents an opportunity to understand, prevent or alleviate a serious problem affecting the health or welfare of children (can be approved with additional safeguards under exceptional circumstances).

Generally speaking, the primary ethical challenge regarding risk is to ensure protection of individual children while making sure research improves the situation of children as a group (Kopelman, 2000). The priority is on avoiding research that is more than minimally risky. The main reason for an exception to this rule is that proposed research has a likelihood of addressing children's need for improved services. Usually, such benefits have to accrue to the children directly; however, under some circumstances research might go ahead that is likely only to benefit other children who suffer from the same condition. This 'subject condition requirement' has been in place since the Belmont report (Department of Health, Education and Welfare, 1979); however, it has been criticised by some authors as problematic under some circumstances (Gordon et al, 1996; Wendler et al, 2003).

Only under very rare circumstances are any exceptions to these rules made, even though in recent years in the USA there has been a significant increase in applications for undergoing a special review process ('407 research') that can allow such exceptions; however, there still exists a lack of clarity about ethical parameters in this review process, relating, for example, to risk thresholds and children's assent requirements (Murphy, 2003; Ross, 2004b). Despite a broad agreement on the basic principles underlying these regulations, there is significant variation in the application of these guidelines, indicating a need for further specification of criteria (Fisher et al, 2007; Gordon et al, 2000; Kon, 2007; Lantos, 2007; Shah et al, 2004; Tauer, 2002; Wendler et al, 2005). International research ethics practices regarding children's research frequently resemble the ones set out in these guidelines; however, a certain variability regarding the ethical criteria exists and some codes promote more restrictive guidelines (Kopelman, 2000).

In the case of clinical trials, one area that lacks clarity is the level of burden that sick children should be allowed to undergo in the context of research. Their experience of routine medical care is often quite burdensome; if they participate in research, one of the questions is whether the standard of minimal risk should be set at what is routine for them or what would be routine for children without their condition. It has been argued that the experience of healthy children should be taken as the minimum risk norm and also that sick children's routine experience should not itself be used to determine a specific level of risk for them, but is primarily relevant in the consent process for allowing them a good understanding of the risks involved (Fisher et al, 2007). With regard to medical research on healthy children, the question of what constitutes minimal risk and what departures from minimal risk are acceptable has been discussed with regard to several interventions, such as experiments involving some level of radiation (Freeman, 1994; Specker and Saenger, 1994).

Another concern arises in the area of environmental health research, where there has been concern about investigating health effects on children of environmental toxins that are already present in their everyday life environment. One intensely discussed case was the Grimes v. Kennedy Krieger Lead Abatement Study, which ended in a controversial and much discussed Court ruling against the conduct of non-beneficial research (Glantz, 2002; Kopelman, 2002; Mastroianni and Kahn, 2002; Paulson, 2006; Shamoo, 2002; Spriggs, 2004 and 2007; Wendler, 2004). While this type of research frequently does not add any additional risk to these children's lives, it may not intervene actively to remove or ameliorate potentially significant risks that are detected in the research process. Such research has been criticised as accepting that researchers may be passive bystanders to harm and also as underestimating or ignoring the significance of issues of human rights, justice and disadvantage in research (Ryan, 2006). On the other hand, public health researchers 
have highlighted the importance of paediatric environmental health research (Lanphear et al, 2006; Merlo et al, 2007) and have voiced concerns about the implications of such rulings for the feasibility of environmental research. Community involvement in the research process has been proposed as one way of addressing these issues (Ryan, 2006). Another issue that has been brought up in the debate is environmental research that involves a genetic component, which can have implications in relation to consent and privacy not just for the children involved in research, but also for their families (Fisher, 2006a and 2006b).

With regard to social and behavioural research, it is also comparatively unclear how to apply this standard of not exceeding risks encountered in children's daily lives. Children may routinely encounter significant challenges and risks in their interactions with others, especially in social contexts like their schools. Unexceptional childhood experiences routinely include being faced with situations in which children experience some level of discomfort, embarrassment, invasion of privacy, and even some degree of risk of bodily or psychological harm. However, even if these are common, it is unclear whether the presence of these risks makes comparable risks in research ethically acceptable (Thompson, 1990). For research in these areas, RECs seem generally inclined to advise against research that involves any additional risk; however, it might be important to raise awareness of the complexity of children's exposure to risk in social and behavioural research (Thompson, 1990).

One area in social research that is traditionally considered to be problematic is the risk involved in deception research (Fisher, 2005). A particularly strong motivation in the research ethics community with regard to social research seems to be to avoid children's confrontation with sensitive subject areas that might cause them discomfort or remind them of negative experiences they have had. The result is frequently that research in these areas is not conducted or significantly modified to allow researchers to avoid these risks. However, perception of risk in these areas might be overstated (Cohn et al, 2005; Denscombe and Aubrook, 1992) and valuable research might be unjustifiably discouraged.

Research with adolescents also encounters particular ethical concerns relating to risk. There is a general perception that adolescents underestimate risks and are particularly prone to risky behaviour that might have significant consequences for their future lives. However, the extent to which this is true, and especially the extent to which this differs from adult self-perceptions, might be overestimated (Millstein and Halpern-Felsher, 2002; Quadrel et al, 1993). Adolescent health research frequently addresses adolescents' health risks due to risky behaviour, such as substance abuse or sexual behaviour. One question in this regard is whether such research could be considered relatively harmless or whether any research in such sensitive areas should be considered as more than minimal risk due to its subject area (Wagener et al, 2004).

Another ethical concern, which is also partly related to concerns about confidentiality in research, relates to the question of what responsibilities researchers have when becoming aware of risky or ethically problematic behaviour in teenagers who participate in their research (Santelli et al, 2005; Scarr, 1994; Underwood, 2005). There is a concern that researchers may either become complicit with such behaviour if it is not addressed or else risk the alienation of teenagers with whom they are conducting the research. It has been suggested that community consultation involving parents and/or teenagers, or establishing formalised 'youth advisory committees' might be ways of ensuring that research involving sensitive subject areas is performed in a manner that important stakeholders consider ethical (Fisher, 2003a; Fisher and Wallace, 2000; Flicker and Guta, 2008; Helgeland, 2005).

However, while risk avoidance is a strong ethical requirement in children's research, the potential benefits arising from research participation are also ethically relevant. Research participation itself is often considered a significant benefit for children, especially in the clinical area where they may receive treatments that might otherwise not be available. Interestingly, there seems to be some evidence that compared with younger children of a similar population, adolescents are under-represented as research subjects in cancer trials, where the benefit of research participation 
is perceived as particularly likely to be beneficial (Bleyer et al, 1997). Where research participation might lead to a potential benefit for participating children (one that would not otherwise be available to them), this potential benefit is often seen to offset risks that they may face in research participation (Spriggs, 2007). Accordingly, ethical issues around children's access to clinical trials are now frequently framed in terms of their right of access to such trials and the problem of exclusion, rather than in terms of the risk involved in their participation (VandenBosch et al, 1999; Yan and Munir, 2004). This shift in emphasis has been controversial in relation to, for example, the danger of overstating the benefits of research (Rosato, 2000; Ross, 2004a; Taylor, 2007a). This is especially relevant when taking into account the situation of parents who are desperate for any intervention that might have some impact on their children's otherwise hopeless health situation; the reality of the chance of benefit however small should not be discounted (Kodish, 2003; Martin and Robert, 2007). Evidence about the factors that influence parental decisions for their children to take part in clinical trials indicates that information is less important than the characteristics of the relationship between doctor and parents. This suggests that emotional factors may be influencing decision-making (Drotar et al, 2004). Accordingly, it has been argued that, especially for clinical trials, the particular format of children's involvement in research decision-making needs to be re-thought and their input needs to be strengthened (Bluebond-Langner et al, 2005).

For research that is not beneficial to children themselves, one concern that has been highlighted in the literature is the motivation of children themselves and their parents to enrol in this kind of research. Especially in clinical trials, researchers, parents and participants stress the potential benefits of research as an important motivating factor for participation (Brody et al, 2005a and 2005b). However, for non-beneficial research the issue arises why facing any risk might be justified and on the basis of what reasons and standards children should be allowed to participate in such research. There is evidence that parents and children are willing to take a certain amount of risk in research for the benefit of others (Wendler and Jenkins, 2008). There has been some debate around the need for truly altruistic motivation for participation in such non-beneficial research. While altruistic motivation is taken for granted in most non-beneficial research with adult participants, it has been debated whether it is appropriate to assume that children are able to be altruistically motivated (Baylis and Downie, 2003; Halila and Lotjonen, 2003; McGee, 2003; Nelson and Reynolds, 2003; Simon et al, 2006). However, there is significant evidence in favour of children's early capacity for altruism (Wolthers, 2006). For children for whom altruistic motivation is in doubt, the question arises whether parents or guardians should be entitled to enrol them in such research at all. Some researchers assume that the absence of likely harm combined with a child's assent should be sufficient to allow their participation in such research.

The issue of remuneration has been considered even more controversial in the case of children, compared to adults. Some authors state categorically that no remuneration should be given over and above expenses for transport costs, based on the assumption of children's vulnerability and the potential that they or their parents might be unduly swayed in their decision to participate if remuneration were made available (Moolchan and Mermelstein, 2002). This argument seems to underlie the prohibition of the use of payment in clinical trials with minors according to the EU Clinical Trials Directive (EU, 2001). One of the main concerns is the issue of justice, insofar as low-income participants might be more swayed by payments (whether substantial or based on the minimum wage model) than participants from higher income backgrounds (Diekema, 2005). However, there is some evidence that participants consider remuneration for participation in biomedical research only as a minor factor in decision-making (Scherer et al, 2005). It has been argued that it is an issue of fairness to give children or young people something back in return for their participation. While small children do not seem to grasp the significance and value of payment, children and adolescents over 9 years of age have a better understanding of what might be appropriate levels of payment (Bagley et al, 2007).

One study that looked at what participants themselves would consider a fair level of payment showed that there were some differences between what researchers and potential participants considered fair (Scherer et al, 2005). In practice, similar to adult research, there is significant 
variation in practices relating to the payment of participants. Ethics committees differ in their views on whether payment should be allowed in children's research at all (Weise et al, 2002). In a review of reported use of payments in research with adolescents, approximately $50 \%$ of researchers used payment (Borzekowski et al, 2003). Many researchers seem to favour providing participants with some token of appreciation with minor monetary value, such as cinema vouchers, pens or T-shirts (Flicker and Guta, 2008). However, it has been argued by some that it might be appropriate to apply a 'minimum wage payment' model not only to adult research participants, but also to research with older children and adolescents (Bagley et al, 2007; Thomas and 0'Kane, 1998). In the case of research with adolescents, there is some evidence that use of payment might be particularly favoured in research with 'at risk' youth (Borzekowski et al, 2003).

\section{Stakeholders' perspectives on risk and benefit in children's research}

In the present study, limited reference was made to the benefits of research as such, although one REC member stated:

'From a benefit point of view, you have to be able to argue that here we are looking at a study that might involve 20 or 30 children, but the benefit is that these findings are going to generalise to a whole number of children in the future. I think you can accept research on children more if that case can be made, that this is the benefit.' (CM)

However, one Chair of an academic REC cautioned:

'I think one other thing is overstated benefit ... How is it [the research] of benefit to you, and very often people are overstating that, [or] ... how is it of benefit to the child? Well, most of the time, it is of no benefit at all to the child. [Yet] it kind of states that it would be.' (AC)

Participants referred to risk and benefit in terms of a relationship between the two, often referring to the need to enable children to take part in research that relates to them (some referring to this as a 'right') and the need to reduce exposure to any risk. Participants consistently referred to 'sensitive' topic areas:

'As someone who works in this area, [one of the difficulties] for me [is] there is a balance to be struck between protecting the child and recognising the personhood of the child. I'd be conscious of that from working with the group. But sometimes the protective can take over. We have had applications for talking to young people about very sensitive topics and I would have felt very strongly that it is their right to be involved in research that affects them. But you do have to balance that with doing it appropriately. Giving them the option to hear their views and protecting them at the same time. That's a difficult balancing act, particularly with very sensitive subjects or particular approaches.' (CM)

This balancing act between risk and benefit was considered difficult for both individual researchers and RECs:

'They are the sort of issues that can be very challenging for an ethics committee, whether they are familiar with child research or not. The balancing of that sense of protection with that right.' (CM)

Some researchers felt that RECs which lacked experience of research with children were particularly disadvantaged when reviewing children's research:

'I didn't feel that there was a lot of experience around the table of research with children and sometimes people felt that the questions we were asking were not appropriate for children who were ... not teenagers ... and that some of the questions would be too difficult for them. And my experience is that children appreciate and respond very well to being asked about their experiences and when it is a sensitive issue, they take it very seriously. And I just felt that in that example, the ethics committee involved was being very cautious about protecting the child and I can understand that. But without any experience of child-centred research and without really knowing how much children can do ... there is still that perspective that children are perhaps unreliable, that it is not appropriate to ask 
them about their experiences, which I would feel very strongly is not the case. If you do it appropriately and sensitively, they absolutely should be involved.' (CR)

One researcher explained the tension between risk and benefit in research with children when potentially beneficial research is not conducted because of perceived risk to participants:

'The problem is here [names specific research] that if you want to help the children that are not being helped now, how will you ever find out how to help them? You kind of come into this vicious circle - you want to help them, but you don't know who they are. How are you going to find out who they are?... I don't know, I still haven't worked out the answer to that.' (CR)

While this balancing act was recognised as challenging, participants considered that research with children was necessary and important:

'They [children] do have a right for their voices to be included in the research and obviously that has to be done taking into account all the appropriate ethical requirements around research with children. But [we must] not ... shy away from doing research with children, about their lives, just because it may be difficult or it may not be as straightforward as if we went and have parents' reports.' (CR)

Risk was considered by participants in the context of children as vulnerable research participants, but also with this vulnerability often compounded by other factors. Risks of taking part in the research were identified:

'With children's [research] in particular, psychological risk is a big element. Children may be scarred by the experience of being involved.' (CM)

But these risks were perceived to be reduced by the approach taken to the child and the supports made available, often during and after the research:

'I suppose what we are doing is interviewing vulnerable families and in particular children. So obviously they are vulnerable anyway, so there's issues there to begin with ... You don't want to make the situation any worse by bringing up issues or raising upsetting things from the past ... You'd always be very conscious of that as a researcher. You try to get the information as delicately as possible, if you have to get that kind of information. And then there's supports, either you organise it or the workers in the [organisation], so that whoever you are talking to is not left in a bad situation after the research. So that we're not just getting what we need and "Thanks very much"'. (CR)

This need for follow-up was stressed by one researcher:

'I'm concerned that even when we have all things covered ethically with kids, sometimes kids get upset and I think there needs to be stronger protocols around making sure they are $0 \mathrm{~K}$ post-research. I'm talking about more than just ringing them the next day. I actually think 6 months down the line, there should be a check-in.' (CR)

This need for quality control for research with children was reiterated and a suggestion made that children's research be overseen by an independent external body:

'It could be the HEA [Higher Education Authority]. In children's social research, certainly the HSE, the 0MC[YA] and the Family Support Agency are the main providers. And you have Barnardo's or the private providers. But there may be some consortium that should be brought together under the auspices of the HEA that would act as a kind of watchdog group ... I know that "Children Count" in the USA have done some work on this.' (CR)

'There are some children's research centres internationally that have looked at this. So there are best practice models perhaps out there.' (CR) 


\section{Parents' perspectives}

The majority of parents in the present study did not feel that their children needed to be direct recipients of any benefits from the research they were involved in:

'It's like the Census really ... it depends where the research is coming from ... More than likely, this is just going to be a small part of something ... and the point of the research is not likely to be to accrue direct benefit to the individual parts in it.' (FP)

Yet it was considered important that the children in the research knew that what they were involved in would benefit others:

'What would be important is the fact that they know what it's about ... it may not directly impinge on them, but it will hopefully benefit something down the road. Once that's explained at the outset ... that there is no expectation for them that this will lead to directly benefiting [them] ... let them feel that in some way they may have an indirect impact on something else ... on other members of the family by participating.' (FP)

Parents felt that involvement in research, approved by themselves and the school, would have a positive effect on their child:

'Make sure the children know that their opinion matters ... that there is a whole group of experts up in Dublin or Galway or wherever, that they are going to look at your opinion and your opinion matters. That would be good for them, even just [as] a confidencebuilding thing.' (FP)

On the other hand, two parents considered that children should benefit directly from their participation:

'I do definitely [think they should benefit] ... I don't think there's any point in the child going though the research and then it suddenly finishes and they are wondering what the hell was that all about.' (P)

Most of the parents accepted that it would be difficult to ensure that research results have a direct benefit in the short term for each participant:

'It would be a bit hopeful that the world is like that ... unfortunately, it might take 10 years.' $(P)$

The issue of asking children uncomfortable questions elicited a variety of responses from parents. A few parents were categorical in their response about ever asking questions that might make a child feel uncomfortable, although the age of the child was considered:

'No. Kids are too young in primary [school] to be introduced to uncomfortable situations.' (P)

Others explored the various circumstances in which it would be reasonable for a child to feel uncomfortable, but only with specific caveats in relation to the child's age, nature of the subject, provision of support and skill of the researcher:

'There are times when it may be reasonable to ask people questions that may be uncomfortable. But again, you have got to look at the age of the participant and the necessity of what is being asked ... I wouldn't rule it out.' (P)

'We need to address the issues and we're not going to find out the explanations if we don't ask the questions, But there needs to be something there. If something, for whatever reason, triggers something difficult for a child, that there is some bit of support readily available.' (P)

'[The] only way you can ask questions on issues like child abuse or whatever is that the child would feel uncomfortable. But it would have to be done in a certain way to minimise the discomfort, which is why the researchers should be trained.' (P) 
Thus, the majority of parents felt that uncomfortable questions may be asked if:

'An ordinary amount of due care and consideration should be used ... You cannot account for every eventuality ... Asking stuff that you know will upset nearly every child would be a no-no. But in things that you don't know, there might be one or two who maybe mightn't like it ... You can't mollycoddle them too much either.' (P)

However, parents acknowledged differences by age group and topic area:

'When asking early teenagers ... they are far more capable of understanding and responding than a 7-year-old. Again, it depends on the question.' (P)

On the subject of young participants in research receiving some kind of remuneration for their time, one mother mentioned the giving-out of pencils as a suitable token for the child's contribution, one that the child could understand and see as being significant.

\section{Children's perspectives}

The participants were divided on whether it was alright to discuss sensitive or difficult topics in research with children. Some participants identified themselves as unsure in relation to this issue: 'You shouldn't if it's going to upset people, but you should if it's going to help people' (C).

Others provided caveats - 'Depends what the situation is' (C) - and recognised that 'Some people might not want to talk about it' (C).

Alternative views were held by others, who considered that 'Better still have to do the subject and get it over with' (C). In talking about such issues, it 'could help them' (C) and they 'might realise that they are not the only ones going through $i t^{\prime}(C)$. Yet conversely, the young people do not want others to get upset as 'they could start crying' (C) or 'feel embarrassed' (C).

As a result of these possible reactions, most of the participants stated that difficult or sensitive issues are better discussed in one-to-one situations: 'It's better to keep some things private'(C). However, one participant identified advantages of being in a group situation and stated: 'If you were in a group, you would be with your friends' (C).

It is clear that the majority of participants displayed altruism, both in their comments and in the discussion of the various points of view. Most of them favoured upsetting a few for the good of the majority: What happens if it makes most people feel happy - then why not make one person feel bad?' (C). Or expressed more succinctly by one participant: 'Sacrifice one lad for 5 lads' (C).

\section{Main concerns}

- Widespread and significant lack of clarity regarding good practice in the informed consent process, especially regarding the scope of authority of children and adolescents.

- Lack of clarity on acceptable risk and its relation to research benefits.

- Altruism as significant motivation for parents' and children's participation in children's research, including a certain degree of psychological risk.

- Lack of clarity on good practice relating to use of incentives for research participation. 


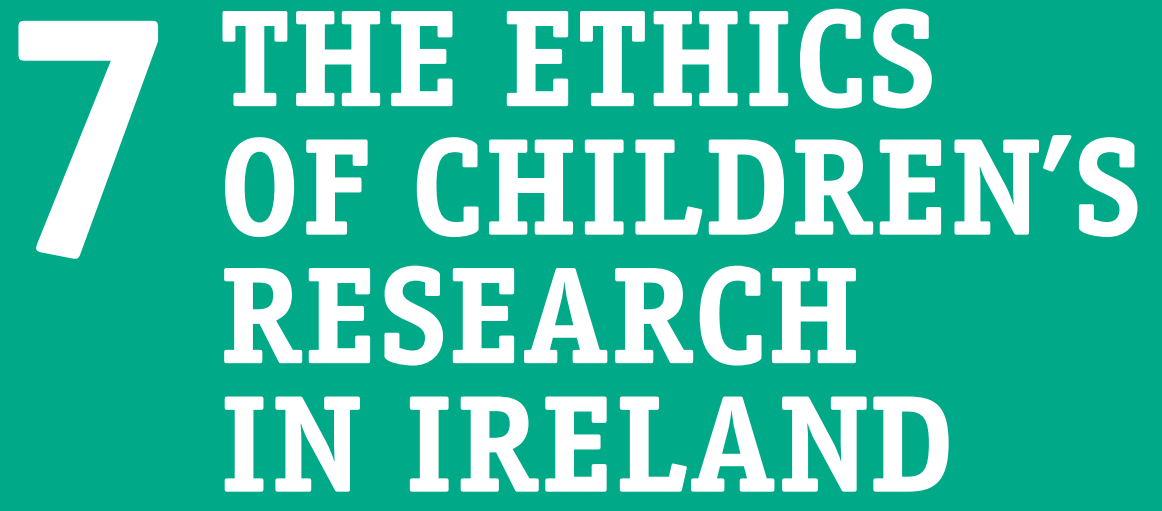




\section{The tensions}

While there is a tradition of highly formalised and professionalised research ethics review procedures in North America, the development of comparative structures has proceeded at a significantly slower pace in Europe and other continents. In Europe, increased attention has been paid to the procedures of Research Ethics Committee (RECs) since the 2002 European Clinical Trials Directive came into effect. Conducting ethical review of research proposals through RECs has become an increasingly important element of the research process in Ireland in recent years. This is reflected in the increase in the number of active RECs, which are at various stages of development with concomitant levels of experience of review processes. As discussed in Chapter 4, RECs in Ireland fall into three main categories: health and social care committees (which include clinical trials committees, for which there is the most structured governing mechanism emanating from the EU), committees based in academic institutions, and others that include committees linked to professional bodies.

Overall, Ireland has been comparatively slow in adopting formalised ethics review procedures. However, the publication of the Operational Procedures for Research Ethics Committees in 2004 by the Irish Council for Bioethics marked the departure for more widespread attention to the implementation of structures and procedures to support good practice (ICB, 2004). While developments appear to be progressing, participants still identify gaps both at the level of overarching governance of RECS and in the provision of independent ethical research review, particularly in relation to some school-based research with children. There is a lack of consistency in relation to operating procedures and review processes between RECs and the range of structural models of RECs in the Irish context is striking. This is seen both within and between the three main categories identified for Irish RECs. Some diversity should be expected in order to facilitate the effective ethical review of the variety of research methodology reported to be undertaken and the array of research settings used. However, the range of structures, in conjunction with the assortment of committee composition, workload, training and actual review processes (such as operating procedures and application processes), suggests that best practice across all RECs will be difficult to achieve. It may be that a national overarching governance structure is required to facilitate all active RECs. The challenge of this approach is the need for such a structure to be able to mediate a number of tensions. One of these tensions is between the need for flexibility, on the one hand, and standardisation of review processes, on the other. This is complicated by the tension between local requirements for ethical review processes in a variety of institutions with standardised international directives for, for example, clinical trials. These tensions are embedded within the context of a dynamic research environment, which accommodates a broad range of research methodologies that are evolving.

While there is diversity in research ethics review, there are also points of commonality. The first is that the primary goal of the ethics review process for all RECs is the protection of research participants. The second is that ethical review, as practised in contemporary RECs, while deliberating a range of ethical issues in research practice, is usually based on the principles of respect for autonomy and dignity, non-maleficence, beneficence and justice. The third commonality is of a more pragmatic nature, in that RECS share the need to develop adequate and effective procedural mechanisms for the review process. Certain ethical issues are given prominence in REC review generally and these include procedures for participant recruitment, informed consent, confidentiality, risk assessment, debriefing or follow-up. In addition, issues of quality assurance are important, such as training of those participating in ethical review and monitoring of processes. These commonalities could form the basis for the beginning of dialogue in the further development of effective and efficient research ethics review in Ireland.

The tensions described above are generic to all research carried out. This includes research with children, which generates further challenges to the ethical review process. These challenges are related to the perceived nature of children, especially to their decision-making capacity, their vulnerability and the particular role of parents or guardians in the research process. 
In relation to children's decision-making capacity, there is a lack of conformity in Irish legislation, identified by participants in the present study, of the chronological age at which young people are considered able to make decisions about their lives independently of their parents. The requirements of informed consent in children is an area of particular concern and challenges and tensions were identified. In terms of research participation, the age of consent was generally cited as 18 years, but this was often perceived as ethically inappropriate. The apparent legislative discrepancy caused some tension, notably when considered in relation to research that was perceived to pose a low risk to older children but that required parental consent. This identifies a potential additional tension in children's research - between children and their parents. Procedures need to do justice to the rights of children and their parents to consent or refuse participation in research, with due regard both to the autonomy, but also to the varying range of levels of maturity and vulnerability of children, and potential cases of disagreement between both. The specific role of children themselves in the consent process should be explored further.

Furthermore, the issue of risk assessment in children's research seems to be a paramount concern for RECs. The strong demand of protecting children from harm needs to be balanced with the equally urgent need for well-founded research that can help improve the lives of children.

The difficulty in navigating these generic and specific tensions and challenges to the ethical review of children's research was identified by stakeholders in the present study, some of whom had suggestions for future developments.

\section{Stakeholders' perspectives for the future}

The need in the future for national guidelines on issues for ethical review processes was identified by participants in the present study:

'If we could have an agreed set of protocols that all schools might sign up to, I think that would be very useful. It is something that I have in my head as something to broach with various bodies.' (AC)

'Something like what the HSE published recently on indemnity - just a 2-page document that made things clear that were previously only in much larger documents and unclear.

Some advice of a legal nature would also make sense. It could be combined with training, an introductory course that covers some basic general issues about REC work and then specifically the 3 Directives on clinical trials, medical devices and local HSE requirements. Another area [for clarification] might be details on consent, especially more clarity on the age of consent for certain issues.' (HC)

The development of coherent national procedures was generally presented as a potentially positive development, with specific reference to training initiatives and operating procedures and processes, including documentation. However, the centralisation of RECs was considered problematic by one Chair of a hospital-based REC:

'I would be totally against that [reducing the number of RECs and centralising review in regional committees]. There is so much work to be done, the workload would be too big and couldn't be done by one committee. There is need for timely decision-making and that just doesn't seem feasible. I don't see the benefit of centralising, especially because with the RECs as they are and the voluntary work that is done by them, it costs nothing for the HSE. If it was centralised, you would have all that travel from the different institutions to the meeting and the costs associated with that. There would need to be good representation of all the relevant institutions and it would just be difficult to organise. And the timeline for reviewing proposals would probably become worse.' (HC)

Interestingly, the proposed model of centralisation was supported by the Chair of one academic REC: 'The concept can be done based on what the European Union was trying to do in relation to hospital approval - that there would be one central medical ethics committee that would approve drug studies, which is meant to be the case, but as we know the hospitals 
won't allow that to occur so they've got their individual committees as well. But there could be a central primary and a central secondary school ethics committee to approve studies ... and that would be made up of teachers and people who are within those remits.' (AC)

How development was managed in one institution was referred to by another Chair of an academic REC:

'The other thing is, the procedures we have at [institution governance level] are not overly detailed and that was a conscious decision. We couldn't over-proceduralise before we had this thing up and running. It should be something that could develop organically, that could be reviewed periodically in the light of lessons learned. I think that's very often the best approach. There is some lack of clarity, there's no question about that, to allow for development, flexibility, to identify what the issues are going to be, to identify how resource-intensive it's going to be. It can be a very difficult business to design a hypothetical regime in the absence of clear experience.' (AC)

In relation to future challenges, the development of research approaches was identified, specifically in relation to research with children:

'I think there is another significant issue and it's not one I have dipped my toe in at all. There is a small body of literature coming from the UK on it. It's the use of children as researchers ... Some would argue that it's a very child-centred approach and it takes away that power status thing between the unknown researcher and the child. It's kind of more equal status. It's when is that appropriate and what support structure should be in place, how do you oversee it and what's your outcome. There's loads of things there. Maybe that is going to be a challenge for future ethics committees. But I'd imagine at the moment it is probably something quite new, not used a lot. But it's out there and it may come our way somewhat more in the future.' (CR)

\section{Conclusion}

With the increase in research, particularly in recent years into the lives of children, the research ethics review landscape in Ireland is continually developing. The majority of Irish RECs identified that they reviewed research with children as part of their function. The diversity and range of research with children reported reflects the range of general research reviewed in every way. Therefore, the generic challenges and tensions identified are applicable to the ethical review of research with children.

The stakeholders' perspectives described above demonstrate clearly some of these tensions and also illustrate the continuing evolution of RECs within the current lack of any agreed national coherent governance framework, which is likely to lead to further divergence. The participants' perspectives also highlight the range of stakeholders in the review process, including schools, healthcare and academic institutions, as well as various professional groups, families and children. It is therefore incumbent on all stakeholders, including children, to voice their respective views as the beginning of a necessary dialogue for the effective ethical review of research with children in Ireland.

\section{Recommendations}

The following concerns have emerged as being in need of attention in order to ensure that children's research in Ireland is conducted ethically and reviewed adequately by RECs. The recommendations in relation to the general research ethics governance system are largely in agreement with previous Irish reports on the issue, with the exception of the assessment of the benefits of a centralised, non-localised review system with a strictly limited number of committees, which was not generally supported by the consulted stakeholders in this study. This difference was likely due to the nature of the research that consulted RECs and other stakeholders engaged in: the 
majority of consulted stakeholders were strongly, if not exclusively, involved in research outside the clinical trials sector, and especially for the children's research stakeholders, a significant proportion of these were working either in social science research or in health research based on social science methodologies.

\section{Creation of central research ethics resource}

The general perception of stakeholders was that there is a need for a central research ethics resource, its functions to include especially the provision of authoritative advice, training and networking for REC members and researchers. In relation to children's research, such a resource would provide information, guidelines and training on the ethical conduct of research with children; details regarding the implementation of these are given in (3) and (4) below.

\section{Comprehensive governance system and national standardisation}

While many participants strongly valued the independence of their own RECs, it was also common for participants to express the view that the existing system was suboptimal in relation to its current lack of governance. The clinical trials REC system was perceived as reasonably functional (despite some significant misgivings on aspects of the system), but the review of other research, especially but not exclusively other types of multi-centre studies, was perceived to be urgently in need of reform.

Those participants who addressed the issue tended to favour a governance model that would provide standardisation of review, without centralisation and loss of review authority by local committees. Nevertheless, they acknowledged the need for extensive standardisation and a governance system that would ensure consistency across RECs. Aspects of such governance identified as desirable included:

- Standardisation of application forms across Irish organisations, allowing for adequate differentiation between research sectors and methodologies.

- Implementation of standardised online submission procedures.

- Standardisation of review procedures, including expedited review, and timelines across Ireland.

- Creation of binding procedures for the review of multi-centre research that does not fall under the Clinical Trials Directive.

- Implementation of clear and consistent appeals procedures within RECs and review feasibility of external adjudication.

- Guidelines for membership, including increased clarity on role of lay members.

- Use of same or compatible software for record-keeping.

- Streamlining the review of multi-centre studies.

In the absence of a governance body with the authority to implement review guidelines, it is urgently required to conduct a consultation regarding the review of multi-centre studies that do not fall under the Clinical Trials Directive, with the goal of coming to an agreement on binding, streamlined review procedures across Ireland. Such a consultation should ideally be conducted across the different sectors - academic, HSE and voluntary.

\section{Creation of suitable structures for the review of children's research}

While very few stakeholders identified as desirable the implementation of completely separate structures for the review of children's research, there was a widespread perception that REC members felt less competent to review children's research compared to research with adults. While general competence-building of REC members in this area of research would be one possible solution, an alternative step could be the implementation of a limited number of RECs with special expertise in children's research, as realised in the UK system. Given concerns about overly narrowing down the scope of review activities of RECS, such RECS could also remain open to the 
review of other types of research. Relevant elements in setting up such specialised structures for review of children's research would include:

- flagging RECs with special expertise in children's research, based on review load and membership;

- standardisation of application forms and documentation requirements for children's research;

- providing training for REC members on such committees in good practice, ethical concerns and common research methodologies in children's research.

\section{Meeting specific information needs}

Many stakeholders mentioned that there was uncertainty, both among REC members and researchers, in relation to the ethical and legal requirements of children's research. This uncertainty can lead to overprotectiveness and insufficient protection of children in research. In light of the importance of safeguarding children's rights, a clear and practical set of workable definitions is needed, especially on the scope for informed consent for mature minors in the Irish context. The following aspects emerged as particularly relevant to stakeholders:

- General guidelines of good practice in children's research and the dissemination of these online and through published leaflets.

- Specific information on:

- legal obligations and relevant documentation, e.g. Children First: National Guidelines for the Protection and Welfare of Children (OMCYA, 1999/2009);

- informed consent and assent procedures, and the creation of information sheets on these;

- confidentiality in children's research;

- acceptable risk.

- Standards for school-based research, including the clarification of the role of Principals, parents and pupils, researchers' responsibilities and the danger of over-researching (i.e. involving the same children or groups of children in too much research - a research burden experienced, for example, by the Traveller community in Ireland).

- Standards for dual role research (e.g. teachers engaging in research activities with their pupils).

- Standards for university student research with children.

- Develop and provide dedicated training modules (online or workshops) on important research ethical issues in children's research.

- Work towards the clarification of the legal requirements of informed consent for mature minors, with particular attention to the adolescent age bracket of 16-18 years.

\section{Involving children in the research process}

While there is relatively broad endorsement of the participatory agenda (i.e. acknowledgment of the need to facilitate children's active engagement in the research process), there is so far no reflection of this in the Irish research ethics review process. As evidenced in the present study and as supported by the international literature, children and young people show a good understanding of ethical concerns in research. Accordingly, their input is of value for:

- the development of material and guidelines on children's research for Irish RECs;

- an exploration of possibilities of involving children and parents in other functions in the design of ethical research, during the research ethics review process or in the research ethics governance system. 
REFERENCES 
Abramovitch, R., Freedman, J.L., Henry, K. and Van Brunschot, M. (1995) 'Children's capacity to agree to psychological research: Knowledge of risks and benefits and voluntariness', Ethics \& Behavior, Vol. 5, No. 1, pp. 25-48.

ACSTI (2006) Towards Better Health: Achieving a Step Change in Health Research in Ireland. Dublin: Advisory Council for Science, Technology and Innovation, and Forfas.

Al-Shahi, R. (2005) 'Research ethics committees in the UK - The pressure is now on research and development departments', Journal of the Royal Society of Medicine, Vol. 98, No. 10, pp. 444-47.

Alderson, P. and Morrow, V. (2004) Ethics, Social Research and Consulting with Children and Young People. Barkingside: Barnardos.

Allard, A. (1996) 'Involving Young People - Empowerment or Exploitation?', Children \& Society, Vol. 10, No. 2, pp. $165-67$.

Allen, G. (2005) 'Research ethics in a culture of risk'. In: A. Farrell (ed.), Ethical Research With Children. New York: Open University Press, pp. 15-26.

Anderson, E.E. (2006) 'A Qualitative Study of Non-Affiliated, Non-Scientist Institutional Review Board Members', Accountability in Research, Vol. 13, No. 2, pp. 135-55.

Anderson, W., Cordner, C.D. and Breen, K.J. (2006) 'Strengthening Australia's framework for research oversight', Medical Journal of Australia, Vol. 184, No. 6, pp. 261-63.

Angell, E., Sutton, A., Windridge, K. and Dixon-Woods, M. (2006) 'Consistency in decision-making by research ethics committees: A controlled comparison', Journal of Medical Ethics, Vol. 32, pp. 662-64

Annas, G.J. (1994) 'Will the real bioethics (commission) please stand up?', The Hastings Center Report, Vol. 24, No. 1, pp. 19-21.

Appelbaum, P.S. (2002) 'Clarifying the Ethics of Clinical Research: A path toward avoiding the therapeutic misconception', The American Journal of Bioethics, Vol. 2, No. 2, pp. 22-23.

Appelbaum, P.S., Lidz, C.W. and Grisso, T. (2004) 'Therapeutic Misconception in Clinical Research: Frequency and Risk Factors', IRB: Ethics \& Human Research, Vol. 26, No. 2, pp. 1-8.

Armstrong, D., Kline-Rogers, E., Jani, S.M., Goldman, E.B., Fang, J., Mukherjee, D., Nallamothu, B.K. and Eagle, K.A. (2005) 'Potential Impact of the HIPAA Privacy Rule on Data Collection in a Registry of Patients With Acute Coronary Syndrome', Archives of Internal Medicine, Vol. 165, No. 10, pp. 1125-29.

Ashcroft, R.E., Goodenough, T., Williamson, E. and Kent, J. (2003) 'Children's Consent to Research Participation: Social context and personal experience invalidate fixed cutoff rules', The American Journal of Bioethics, Vol. 3, No. 4, pp. 16-18.

Australia, Government of (2007) Budget 2007/2008: Health and medical research - Streamlining human research ethics reviews. Canberra: Government of Australia. Available at: www.health.gov.au/internet/budget/publishing.nsf/Content/A1 8BA291FEACC14BCA2572D40028A1F7/\$File/hfact46.pdf (accessed February 2010)

Azar, B. (2002) 'Ethics at the cost of research?', Monitor on Psychology, Vol. 33, No. 2, pp. 38-40.

Bagley, S.J., Reynolds, W.W. and Nelson, R.M. (2007) 'Is a "Wage-Payment" Model for Research Participation Appropriate for Children?', Pediatrics, Vol. 119, No. 1, pp. 46-51.

Balen, R., Blyth, E., Calabretto, H., Fraser, C., Horrocks, C. and Manby, M. (2006) 'Involving Children in Health and Social Research: "Human becomings" or "active beings"?', Childhood, Vol. 13, No. 1, pp. 29-48.

Bamberg, M. and Budwig, N. (1992) 'Therapeutic Misconceptions: When the voices of caring and research are misconstrued as the voice of curing', Ethics \& Behavior, Vol. 2, No. 3, pp. 165-84.

Bankert, E. and Amdur, R. (2000) 'The IRB is not a data and safety monitoring board', IRB: Ethics and Human Research, Vol. 22, No. 6, pp. 9-11.

Barker, J. and Smith, F. (2001) 'Power, Positionality and Practicality: Carrying out Fieldwork with Children', Ethics, Place \& Environment, Vol. 4, No. 2, pp. 142-47.

Baylis, F. and Downie, J. (2003) 'The Limits of Altruism and Arbitrary Age Limits', American Journal of Bioethics, Vol. 3, No. 4, pp. 19-21.

Baylis, F., Downie, J. and Kenny, N. (1999) 'Children and Decision-making in Health Research', IRB: Ethics and Human Research, Vol. 21, No. 4, pp. 5-10.

BERA (2004) Revised Ethical Guidelines for Educational Research (2004). Southwell, Notts: British Educational Research Association.

Berg, L.D., Evans, M., Fuller, D. and the Okanagan Urban Aboriginal Health Research Collective (2007) 'Ethics, Hegemonic Whiteness, and the Contested Imagination of "Aboriginal Community" in Social Science Research in Canada', ACME: An International E-Journal for Critical Geographies, Vol. 6, No. 3, pp. 395-410.

Bhattacharya, K. (2007) 'Consenting to the Consent Form: What are the fixed and fluid understandings between the researcher and the researched?', Qualitative Inquiry, Vol. 13, No. 8, pp. 1095-1115.

Biehl, M. and Halpern-Felsher, B.L. (2001) 'Adolescents' and adults' understanding of probability expressions', Journal of Adolescent Health, Vol. 28, No. 1, pp. 30-35.

Blake, M.K. (2007) 'Formality and Friendship: Research Ethics Review and Participatory Action Research', ACME: An International E-Journal for Critical Geographies, Vol. 6, No. 3, pp. 411-21.

Bleyer, W.A., Tejeda, H., Murphy, S.B., Robison, L.L., Ross, J.A., Pollock, B.H., Severson, R., Brawley, 0., Smith, M. and Ungerleider, R. (1997) 'National cancer clinical trials: Children have equal access; adolescents do not', Journal of Adolescent Health, Vol. 21, No. 6, pp. 366-73.

Bluebond-Langner, M., DeCicco, A. and Belasco, J. (2005) 'Involving children with life-shortening illnesses in decisions about participation in clinical research: A proposal for shuttle diplomacy and negotiation'. In: E. Kodish (ed.), Ethics and Research with Children: A Case-based Approach. New York: 0xford University Press, pp. 323-43.

Bogolub, E. B. and Thomas, N. (2005) 'Parental Consent and the Ethics of Research with Foster Children: Beginning a Cross-Cultural Dialogue', Qualitative Social Work, Vol. 4, No. 3, pp. 271-92.

Bortolussi, R. and Nicholson, D. (2002) 'Auditing of clinical research ethics in a children's and women's academic hospital', Clinical and Investigative Medicine, Vol. 25, No. 3, pp. 83-88. 
Borzekowski, D.L.G., Rickert, V.I., Ipp, L. and Fortenberry, J.D. (2003) 'At what price? The current state of subject payment in adolescent research', Journal of Adolescent Health, Vol. 33, No. 5, pp. 378-84.

Boser, S. (2007) 'Power, Ethics and the IRB: Dissonance over human participant review of participatory research', Qualitative Inquiry, Vol. 13, No. 8, pp. 1060-74.

Bosk, C.L. (2007) 'The New Bureaucracies of Virtue or when form fails to follow function', PoLAR Political and Legal Anthropology Review, Vol. 30, No. 2, pp. 192-209.

Bostock, L. (2002) ' “God, She's gonna report me": The Ethics of Child Protection in Poverty Research', Children \& Society, Vol. 16, No. 4, pp. 273-83.

Botkin, J.R. (2003) 'Preventing Exploitation in Pediatric Research', The American Journal of Bioethics, Vol. 3, No. 4, pp. 31-32.

Boulton, M., Brown, N., Lewis, G. and Webster, A. (2004) Implementing the ESRC Research Ethics Framework: The Case for Research Ethics Committees. York: Economic and Social Research Council.

Bradley, M. (2007) 'Silenced for Their Own Protection: How the IRB marginalizes those it feigns to protect', ACME: An International E-Journal for Critical Geographies, Vol. 6, No. 3, pp. 339-49.

Brandt, A.M. and Freidenfelds, L. (1996) 'Research Ethics after World War II: The Insular Culture of Biomedicine', Kennedy Institute of Ethics Journal, Vol. 6, No. 3, pp. 239-43.

Bray, L. (2007) 'Developing an activity to aid informed assent when interviewing children and young people', Journal of Research in Nursing, Vol. 12, No. 5, pp. 447-57.

Breese, P., Rietmeijer, C. and Burman, W. (2007) 'Content among locally approved HIPAA Authorization forms for Research', Journal of Empirical Research on Human Research Ethics, Vol. 2, No. 1, pp. 43-46.

Breese, P.E., Burman, W.J., Goldberg, S. and Weis, S.E. (2007) 'Education Level, Primary Language and Comprehension of the Informed Consent Process', Journal of Empirical Research on Human Research Ethics, Vol. 2, No. 4, pp. 69-79.

Brody, J.L., Annett, R.D. and Scherer, D.G. (2003a) 'Adolescent, parent and physician views on biomedical research participation', Journal of Adolescent Health, Vol. 32, No. 2, p. 122.

Brody, J.L., Annett, R.D., Scherer, D.G., Perryman, M.L. and Cofrin, K.M.W. (2005a) 'Comparisons of adolescent and parent willingness to participate in minimal and above-minimal risk pediatric asthma research protocols', Journal of Adolescent Health, Vol. 37, No. 3, pp. 229-35.

Brody, J.L., Scherer, D.G. and Annett, R.D. (2005b) 'Factors influencing adolescent and parent decisions to enroll in a randomized placebo controlled asthma research trial', Journal of Adolescent Health, Vol. 36, No. 2, p. 105.

Brody, J.L., Scherer, D.G., Annett, R.D. and Pearson-Bish, M. (2003b) 'Voluntary Assent in Biomedical Research With Adolescents: A Comparison of Parent and Adolescent Views', Ethics \& Behavior, Vol. 13, No. 1, pp. 79-95.

Brooks-Gunn, J. and Rotheram-Borus, M.J. (1994) 'Rights to Privacy in Research: Adolescents versus Parents', Ethics \& Behavior, Vol. 4, No. 2, pp. 109-21.

Broome, M.E. (1999) 'Consent (assent) for research with pediatric patients', Seminars in Oncology Nursing, Vol. 15, No. 2, pp. 96-103.

Broome, M.E. and Richards, D.J. (2003) 'The influence of relationships on children's and adolescents' participation in research', Nursing Research, Vol. 52, No. 3, pp. 191-97.

Broome, M.E., Kodish, E., Geller, G. and Siminoff, L.A. (2003) 'Children in Research: New Perspectives and Practices for Informed Consent', IRB: Ethics and Human Research, Vol. 25, No. 5 (Supplement), pp. S20-S25.

Brown, J., Ryland, I., Howard, J. and Shaw, N. (2007) 'Views of National Health Service (NHS) Ethics Committee members on how education research should be reviewed', Medical Teacher, Vol. 29, No. 2-3, pp. 225-30.

Brown, J.S., Schonfeld, T.L. and Gordon, B.C. (2006) ' "You may have already won!": An examination of the use of lottery payments in research', IRB: Ethics \& Human Research, Vol. 28, No. 1, pp. 12-16.

Bruzzese, J.-M. and Fisher, C.B. (2003) 'Assessing and Enhancing the Research Consent Capacity of Children and Youth', Applied Developmental Science, Vol. 7, No. 1, pp. 13-26.

Burke, T.K. (2007) 'Providing Ethics a Space on the Page: Social Work and Ethnography as a Case in Point', Qualitative Social Work, Vol. 6, No. 2, pp. 177-95.

Burke, T.M., Abramovitch, R. and Zlotkin, S. (2005) 'Children's understanding of the risks and benefits associated with research', Journal of Medical Ethics, Vol. 31, No. 12, pp. 715-20.

Burman, W.J., Reves, R.R., Cohn, D.L. and Schooley, R.T. (2001) 'Breaking the Camel's Back: Multicenter Clinical Trials and Local Institutional Review Boards', Annals of Internal Medicine, Vol. 134, No. 2, pp. 152-57.

Burris, S. and Moss, K. (2006) 'US Health Researchers review their Ethics Review Boards: A Qualitative Study', Journal of Empirical Research on Human Research Ethics, Vol. 1, No. 2, pp. 39-58.

Bushin, N. (2007) 'Interviewing with Children in their Homes: Putting Ethical Principles into Practice and Developing Flexible Techniques', Children's Geographies, Vol. 5, No. 3, pp. 235-51.

Cahill, C. (2007) 'Repositioning Ethical Commitments: Participatory Action Research as a Relational Praxis of Social Change', ACME: An International E-Journal for Critical Geographies, Vol. 6, No. 3, pp. 360-73.

Cahill, C., Sultana, F. and Pain, R. (2007) 'Participatory Ethics: Politics, Practices, Institutions', ACME: An International E-Journal for Critical Geographies, Vol. 6, No. 3, pp. 304-18.

Campbell, E., Weissman, J., Clarridge, B., Yucel, R., Causino, N. and Blumenthal, D. (2003) 'Characteristics of Medical School Faculty Members serving on Institutional Review Boards: Results of a National Survey', Academic Medicine, Vol. 78, No. 8, pp. 831-36.

Candib, L.M. (2007) 'How turning a QI Project into "Research" almost sank a great program', The Hastings Center Report, Vol. 37, No. 1, pp. 26-30.

Cannella, G.S. (2004) 'Regulatory Power: Can a Feminist Poststructuralist engage in Research Oversight?', Qualitative Inquiry, Vol. 10, No. 2, pp. 235-45. 
Cannella, G.S. and Lincoln, Y.S. (2007) 'Predatory vs. Dialogic Ethics: Constructing an Illusion or Ethical Practice as the Core of Research Methods', Qualitative Inquiry, Vol. 13, No. 3, pp. 315-35.

Casarett, D., Karlawish, J.H.T. and Sugarman, J. (2000) 'Determining when quality improvement initiatives should be considered research: Proposed criteria and potential implications', Journal of the American Medical Association (JAMA), Vol. 238, No. 17, pp. 2275-80.

Cauffman, E. and Steinberg, L. (1995) 'The cognitive and affective influences on adolescent decision-making', Temple Law Review, Vol. 68, pp. 1763-89.

Cave, E. and Holm, S. (2002) 'New governance arrangements for research ethics committees: Is facilitating research achieved at the cost of participants' interest', Journal of Medical Ethics, Vol. 28, No. 5, pp. 318-21.

CCPH (2007) Educational Conference Call Series on Institutional Review Boards (IRBs) and Ethical Issues in Research, facilitated by Community Campus Partnerships for Health and Tuskegee University National Center for Bioethics in Research and Health Care. Available at: http://depts.washington.edu/ccph/irbcalls2.html (accessed February 2010)

Central Office for Research Ethics Committees (2004) The ethical governance and regulation of student projects: A draft proposal, Working Group on Ethical Review of Student Research in the NHS. London: Central Office for Research Ethics Committees.

Central Office for Research Ethics Committees (2006) Building on improvement: Implementing the recommendations of the report of the ad hoc advisory group on the operation of NHS research ethics committees. London: Central Office for Research Ethics Committees.

Chalmers, D. and Pettit, P. (1998) 'Towards a consensual culture in the ethical review of research', Medical Journal of Australia, Vol. 169, pp. 79-82.

Choo, V. (1998) 'Thin line between research and audit', The Lancet, Vol. 352, No. 9125, pp. 337-38.

Christensen, P.I.A. and Prout, A. (2002) 'Working with Ethical Symmetry in Social Research with Children', Childhood, Vol. 9, No. 4, pp. 477-97.

Christian, M.C., Goldberg, J.L., Killen, J., Abrams, J.S., McCabe, M.S., Mauer, J.K. and Wittes, R.E. (2002) 'A Central Institutional Review Board for Multi-Institutional Trials', New England Journal of Medicine, Vol. 346, No. 18, pp. $1405-08$.

CIHR, NSERC and SSHRC (1998/2005) Tri-Council Policy Statement: Ethical Conduct for Research involving Humans (1998, with 2000, 2002, 2005 amendments). Ottawa: Interagency Secretariat on Research Ethics, on behalf of the Canadian Institutes of Health Research, Natural Sciences and Engineering Research Council of Canada and the Social Sciences and Humanities Research Council of Canada.

Cocks, A.J. (2006) 'The Ethical Maze: Finding an inclusive path towards gaining children's agreement to research participation', Childhood, Vol. 13, No. 2, pp. 247-66.

Cohn, J.M., Ginsburg, K.R., Kassam-Adams, N. and Fein, J.A. (2005) 'Adolescent Decisional Autonomy regarding Participation in an Emergency Department Youth Violence Interview', The American Journal of Bioethics, Vol. 5, No. 5, pp. 70-74.

Coleman, B.S. (1982) 'The Availability of Independent IRBs', IRB: Ethics and Human Research, Vol. 4, No. 3, p. 10.

Collogan, L. and Fleischman, A. (2005) 'Adolescent Research and Parental Permission'. In: E. Kodish (ed.), Ethics and Research with Children: A Case-based Approach. New York: 0xford University Press, pp. 77-99.

Connolly, K. and Reid, A. (2007) 'Ethics Review for Qualitative Inquiry: Adopting a values-based, facilitative approach', Qualitative Inquiry, Vol. 13, No. 7, pp. 1031-47.

Cooper, M. (2007) 'Sharing Data and Results in Ethnographic Research: Why this should not be an ethical imperative', Journal of Empirical Research on Human Research Ethics, Vol. 2, No. 1, pp. 3-19.

Corbin, J. and Morse, J.M. (2003) 'The Unstructured Interactive Interview: Issues of reciprocity and risks when dealing with sensitive topics'. Qualitative Inquiry, Vol. 9, No. 3, pp. 335-54.

Corrigan, 0. (2003) 'Empty ethics: The problem with informed consent', Sociology of Health \& Illness, Vol. 25, No. 7, pp. 768-92.

Council of Europe (1997) Convention for the Protection of Human Rights and Dignity of the Human Being with regard to the Application of Biology and Medicine: Convention on Human Rights and Biomedicine, Oviedo, 4 April 1997, European Treaty Series No. 164. Strasbourg: Council of Europe. Available at: http://conventions.coe.int/Treaty/en/Treaties/ Html/164.htm (accessed February 2010)

Cox, K. (2000) 'Enhancing cancer clinical trial management: Recommendations from a qualitative study of trial participants' experiences', Psycho-Oncology, Vol. 9, No. 4, pp. 314-22.

Cox, K. (2002) 'Informed consent and decision-making: Patients' experiences of the process of recruitment to Phases I and II anti-cancer drug trials', Patient Education and Counseling, Vol. 46, No. 1, pp. 31-38.

Coyne, I.T. (1998) 'Researching children: Some methodological and ethical considerations', Journal of Clinical Nursing, Vol. 7, No. 5, pp. 409-16.

Cretin, S., Keeler, E.B., Lynn, J., Batalden, P.B., Berwick, D.M. and Bisognano, M. (2000) 'Should patients in qualityimprovement activities have the same protections as participants in research studies?', Journal of the American Medical Association (JAMA), Vol. 284, No. 14, pp. 1786-88.

Crigger, B.-J. (1996) 'Guidelines for Adolescent Health Research', IRB: Ethics and Human Research, Vol. 18, No. 1, pp. 10-11.

Croft, J.R., Festinger, D.S., Dugosh, K.L., Marlowe, D.B. and Rosenwasser, B.J. (2007) ‘Does Size Matter?: Salience of followup payments in drug abuse research', IRB: Ethics \& Human Research, Vol. 29, No. 4, pp. 15-19.

Cromer, L.D., Freyd, J.J., Binder, A.K., DePrince, A.P. and Becker-Blease, K. (2006) 'What's the risk in asking? Participant reaction to trauma history questions compared with reaction to other personal questions'. Ethics \& Behavior, Vol. 16, No. 4, pp. 347-62.

Cummins, H.A. (2006) 'A funny thing happened on the way to the Ethics Board: Studying the meaning of farm life for farm children', Journal of Academic Ethics, Vol. 4, pp. 175-88.

Cuskelly, M. (2005) 'Ethical inclusion of children with disabilities in research'. In: A. Farrell (ed.), Ethical Research with Children. New York: Open University Press, pp. 97-111. 
Dane, F.C. and Parish, D.C. (2006) 'Ethical Issues in Registry Research: In-Hospital Resuscitation as A Case Study', Journal of Empirical Research on Human Research Ethics, Vol. 1, No. 4, pp. 69-76.

Darvall, L. (1995) 'Increased Review Responsibilities for Australian Institutional Ethics Committees', IRB: Ethics and Human Research, Vol. 17, No. 1, p. 9.

Dash, L. (2007) 'Journalism and Institutional Review Boards', Qualitative Inquiry, Vol. 13, No. 6, pp. 871-74.

Daugherty, C., Ratain, M.J., Grochowski, E., Stocking, C., Kodish, E., Mick, R. and Siegler, M. (1995) 'Perceptions of cancer patients and their physicians involved in Phase I trials', Journal of Clinical Oncology, Vol. 13, No. 5, pp. 1062-72.

Daugherty, C.K. (1999) 'Impact of Therapeutic Research on Informed Consent and the Ethics of Clinical Trials: A Medical Oncology Perspective', Journal of Clinical Oncology, Vol. 17, No. 5, pp. 1601-17.

David, M., Edwards, R. and Alldred, P. (2001) 'Children and School-based Research: "Informed consent" or "educated consent"?', British Educational Research Journal, Vol. 27, No. 3, pp. 347-65.

Dawson, A.J. (2005) 'The Ad Hoc Advisory Group's proposals for research ethics committees: A mixture of the timid, the revolutionary and the bizarre', Journal of Medical Ethics, Vol. 31, No. 8, pp. 435-36.

Dawson, A.J. and Yentis, S.M. (2007) 'Contesting the science/ethics distinction in the review of clinical research', Journal of Medical Ethics, Vol. 33, No. 3, pp. 165-67.

Denham, E.J. and Nelson, R. (2002) 'Self-determination is not an appropriate model for understanding parental permission and child assent', Aneasthesia and Analgesia, Vol. 94, No. 5, pp. 1049-51.

Denmark, Government of (2003) Act on a Biomedical Research Ethics Committee System and the Processing of Biomedical Research Projects. Copenhagen: Folketinget.

Denscombe, M. and Aubrook, L. (1992) ' “It's just another piece of schoolwork": The ethics of questionnaire research on pupils in schools', British Educational Research Journal, Vol. 18, No. 2, pp. 113-31.

Department of Health, UK (2005) Report of the Ad Hoc Advisory Group on the Operation of NHS Research Ethics Committees. London: Department of Health.

Department of Health and Children (2004) European Communities (Clinical Trials on Medicinal Products for Human Use) Regulations, 2004. S.I. No. 190 of 2004. Dublin: The Stationery Office.

Department of Health and Children (2007) Guidance on the Application for Recognised Ethics Committee Opinion and the Ethical Review of Clinical Trials on Medicinal Products for Human Use (revised edition July 2007). Dublin: The Stationery Office.

Department of Health, Education and Welfare, US (1979) Belmont Report: Ethical Principles and Guidelines for the Protection of Human Subjects of Research, Report of the National Commission for the Protection of Human Subjects of Biomedical and Behavioral Research. Washington, DC: US Department of Health, Education and Welfare.

Derse, A.R. (1997) 'Whither ethics committee networks?', The Hastings Center Report, Vol. 27, No. 3, p. 47.

De Wolf, V.A., Sieber, J.E., Steel, P.M. and Zarate, A.0. (2005) 'Part I: What is the requirement for data sharing?', IRB: Ethics \& Human Research, Vol. 27, No. 6, pp. 12-16.

De Wolf, V.A., Sieber, J.E., Steel, P.M. and Zarate, A.0. (2006a) 'Part II: HIPAA and disclosure risk issues', IRB: Ethics \& Human Research, Vol. 28, No. 1, pp. 6-11.

De Wolf, V.A., Sieber, J.E., Steel, P.M. and Zarate, A.0. (2006b) 'Part III: Meeting the challenge when data sharing is required', IRB: Ethics \& Human Research, Vol. 28, No. 2, pp. 10-15.

Dickenson, D. (2006) 'Gender and Ethics Committees: Where's the different voice?', Bioethics, Vol. 20, No. 3, pp. 115-24.

Diekema, D. (2005) 'Payments for participation of children in research'. In: E. Kodish (ed.), Ethics and Research with Children: A Case-based Approach. New York: 0xford University Press, pp. 143-60.

Dingwall, R. (2007) The Ethical Case against Ethics Regulation in the Humanities and Social Sciences, 1st Annual Lecture, Humanities Research Centre. Available at: www.nottingham.ac.uk/hrc/documents/dingwall2007.pdf (accessed February 2010)

Dinsdale, H. (2005) 'Professional responsibility and the protection of human subjects of research in Canada', Health Law Review, Vol. 13, No. 2-3, pp. 80-85.

Diviak, K.R., Curry, S.J., Emery, S.L. and Mermelstein, R.J. (2004) 'Human participants challenges in youth tobacco cessation research: Researchers' perspectives', Ethics \& Behavior, Vol. 14, No. 4, pp. 321-34.

Dixon-Woods, M., Angell, E., Ashcroft, R.E. and Bryman, A. (2007) 'Written work: The social functions of Research Ethics Committee letters', Social Science \& Medicine, Vol. 65, No. 4, pp. 792-802.

Dorn, L.D., Susman, E.J. and Fletcher, J.C. (1995) 'Informed consent in children and adolescents: Age, maturation and psychological state', Journal of Adolescent Health, Vol. 16, No. 3, pp. 185-90.

Downie, J. (2006) 'The Canadian Agency for the Oversight of Research involving Humans: A Reform Proposal', Accountability in Research, Vol. 13, No. 1, pp. 75-100.

Doyal, L. (1997) 'Informed consent in medical research: Journals should not publish research to which patients have not given fully informed consent - with three exceptions', British Medical Journal, Vol. 314, No. 7087, pp. 1107-11.

Doyal, L. (2004) 'Preserving moral quality in research, audit and quality improvement', Quality \& Safety in Health Care, Vol. 13, No. 1, pp. 11-12.

Drotar, D., Miller, V., Willard, V., Anthony, K. and Kodish, E. (2004) 'Correlates of parental participation during informed consent for randomized clinical trials in the treatment of childhood leukemia', Ethics \& Behavior, Vol. 14, No. 1, pp. 1-15.

Dyer, S. (2004) 'Rationalising public participation in the health service: The case of Research Ethics Committees', Health \& Place, Vol. 10, No. 4, pp. 339-48.

Easa, D., Kim, K., Kato, K., Ho, T., Balaraman, V., Medina, L., Hammatt, Z. and Shomaker, T.S. (2006) 'The Research Subject Advocate at The University of Hawaii Clinical Research Center: An added resource for protection of human subjects', Hawaii Medical Journal, Vol. 65, No. 2, pp. 50-52.

Easa, D., Norris, K., Hammatt, Z., Kim, K., Hernandez, E., Kato, K., Balaraman, V., Ho, T. and Shomaker, S. (2005) ‘The Research Subject Advocate at Minority Clinical Research Centers: An added resource for protection of human subjects', Ethnicity and Disease, Vol. 15, No. 4 (Supplement 5), pp. 107-10. 
Easter, M.M., Davis, A.M. and Henderson, G.E. (2004) 'Confidentiality: More than a linkage file and a locked drawer', IRB: Ethics \& Human Research, Vol. 26, No. 2, pp. 13-17.

Edwards, R. and Alldred, P.A.M. (1999) 'Children and Young People's Views of Social Research: The Case of research on home-school relations', Childhood, Vol. 6, No. 2, pp. 261-81.

Edwards, S., Stone, T. and Swift, T. (2007) 'Differences between research ethics committees', International Journal of Technology Assessment in Health Care, Vol. 23, pp. 17-23.

Edwards, S.J.L. (2006) 'Restricted Treatments, Inducement and Research Participation', Bioethics, Vol. 20, No. 2, pp. 77-91.

Elliott, L. and Hunter, D. (2008) 'The experiences of Ethics Committee Members: Contradictions between individuals and committees', Journal of Medical Ethics, Vol. 34, pp. 489-94.

Ells, C. and Gutfreund, S. (2006) 'Myths about Qualitative Research and the Tri-Council Policy Statement', The Canadian Journal of Sociology, Vol. 31, No. 3, pp. 361-73.

Elwood, S. (2007) 'Negotiating Participatory Ethics in the midst of Institutional Ethics', ACME: An International E-Journal for Critical Geographies, Vol. 6, No. 3, pp. 329-38.

Emanuel, E. and Grady, C. (2007) 'Four Paradigms of Clinical Research and Research Oversight', Cambridge Quarterly of Healthcare Ethics, Vol. 16, No. 1, pp. 82-96.

Emanuel, E.J., Lemmens, T. and Elliot, C. (2006) 'Should society allow research ethics boards to be run as for-profit enterprises?', PLoS Medicine, Vol. 3, No. 7, e309.

Emanuel, E.J., Wood, A., Fleischman, A., Bowen, A., Getz, K.A., Grady, C., Levine, C., Hammerschmidt, D.E., Faden R., Eckenwiler, L., Muse, C.T. and Sugarman, J. (2004) 'Oversight of Human Participants Research: Identifying problems to evaluate reform proposals', Annals of Internal Medicine, Vol. 141, No. 4, pp. 282-91.

English, A. and Ford, C.A. (2007) 'More evidence supports the need to protect confidentiality in adolescent health care', Journal of Adolescent Health, Vol. 40, No. 3, pp. 199-200.

ESRC (2006) Research Ethics Framework (REF). Swindon, UK: Economic and Social Research Council.

ESRC (2007a) Ethics and Ethical Practice in Social Science Research: Three-day Course on Ethics and Research with "Vulnerable" Groups, Faculty of Arts and Social Sciences, Lancaster University. Swindon, UK: Economic and Social Research Council. Available at: www.lancs.ac.uk/fass/events/ethicalresearch/events/event4.htm (accessed February 2010)

ESRC (2007b) Ethics and Ethical Practice in Social Science Research: Three Day Course on Ethics in Participatory Research, Faculty of Arts and Social Sciences, Lancaster University. Swindon, UK: Economic and Social Research Council. Available at: www.lancs.ac.uk/fass/events/ethicalresearch/events/event5.htm (accessed February 2010)

Essen, G.V., Story, D., Poustie, S., Griffiths, M. and Marwood, C. (2004) 'Natural justice and human research ethics committees: An Australia-wide survey', Medical Journal of Australia, Vol. 180, pp. 63-66.

Estlin, E.J. and Ablett, S. (2001) 'Practicalities and ethics of running clinical trials in paediatric oncology - The UK experience', European Journal of Cancer, Vol. 37, No. 11, pp. 1394-1401.

Etherington, K. (2007) 'Ethical Research in Reflexive Relationships', Qualitative Inquiry, Vol. 13, No. 5, pp. 599-616.

EU (2001) Directive 2001/20/EC of the European Parliament and of the Council of 4 April 2001 on the Approximation of the Laws, Regulations and Administrative Provisions of the Member States relating to the Implementation of Good Clinical Practice in the Conduct of Clinical Trials on Medicinal Products for Human Use, 2001/20/EC. Brussels: European Commission.

Evans, B.J. (2004) 'Inconsistent Regulatory Protection under the US Common Rule', Cambridge Quarterly of Healthcare Ethics, Vol. 13, No. 4, pp. 366-79.

Faden, R.R., Kass, N.E., Klag, M.J. and Krag, S.S. (2002) 'On the importance of Research Ethics and Mentoring', The American Journal of Bioethics, Vol. 2, No. 4, pp. 50-51.

Faden, R.R., Lewis, C. and Rimer, B. (1980) 'Monitoring Informed Consent Procedures: An Exploratory Record Review', IRB: Ethics and Human Research, Vol. 2, No. 8, pp. 9-10.

Farrell, A. (2005) 'Ethics and research with children'. In: A. Farrell (ed.), Ethical Research with Children. New York: Open University Press, pp. 1-14.

Federman, D.D., Hanna, K.E. and Rodriguez, L.L. (eds.) (2002) Responsible Research: A Systems Approach to protecting Research Participants. Washington, DC: The National Academies Press.

Fernandez, C.V. (2003) 'Context in shaping the ability of a child to assent to research', American Journal of Bioethics, Vol. 3, No. 4, pp. 29-30.

FHI (2001) Research Ethics Training Curriculum for Scientists. Research Triangle Park, NC: Family Health International. Available at: www.fhi.org/training/en/Retc/ (accessed February 2010)

Fisher, C.B. (1994) 'Reporting and Referring Research Participants: Ethical challenges for investigators studying children and youth', Ethics \& Behavior, Vol. 4, No. 2, pp. 87-95.

Fisher, C.B. (2003a) 'Adolescent and Parent Perspectives on Ethical Issues in Youth Drug Use and Suicide Survey Research', Ethics \& Behavior, Vol. 13, No. 4, pp. 303-32.

Fisher, C.B. (2003b) 'A Goodness-of-Fit Ethic for Child Assent to Non-beneficial Research', The American Journal of Bioethics, Vol. 3, No. 4, pp. 27-28.

Fisher, C.B. (2005) 'Deception Research involving Children: Ethical practices and paradoxes', Ethics \& Behavior, Vol. 15, No. 3, pp. 271-87.

Fisher, C.B. (2006a) 'Privacy and Ethics in Pediatric Environmental Health Research. Part I: Genetic and Prenatal Testing', Environmental Health Perspectives, Vol. 114, No. 10, pp. 1617-21.

Fisher, C.B. (2006b) 'Privacy and Ethics in Pediatric Environmental Health Research. Part II: Protecting Families and Communities', Environmental Health Perspectives, Vol. 114, No. 10, pp. 1622-25.

Fisher, C.B., Kornetsky, S.Z. and Prentice, E.D. (2007) 'Determining Risk in Pediatric Research with No Prospect of Direct Benefit: Time for a national consensus on the interpretation of federal regulations', The American Journal of Bioethics, Vol. 7, No. 3, pp. 5-10. 
Fisher, C.B. and Wallace, S.A. (2000) 'Through the Community Looking Glass: Re-evaluating the ethical and policy implications of research on adolescent risk and psychopathology', Ethics \& Behavior, Vol. 10, No. 2, pp. 99-118.

Fisher, J.A. (2006) 'Procedural Misconceptions and Informed Consent: Insights from Empirical Research on the Clinical Trials Industry', Kennedy Institute of Ethics Journal, Vol. 16, No. 3, pp. 251-68.

Fitzgerald, M.H. and Phillips, P.A. (2006) 'Centralized and Non-centralized Ethics Review: A Five Nation Study', Accountability in Research, Vol. 13, No. 1, pp. 47-74.

Fitzgerald, M.H., Phillips, P.A. and Yule, E. (2006) 'The Research Ethics Review Process and Ethics Review Narratives', Ethics \& Behavior, Vol. 16, No. 4, pp. 377-95.

Flagel, D.C. (2000) 'Children as Research Subjects: New Guidelines for Canadian IRBs', IRB: Ethics and Human Research, Vol. 22, No. 5, pp. 1-3.

Fleischman, A.R. (2005) 'Regulating Research with Human Subjects - Is the system broken?', Transactions of the American Clinical and Climatological Association, Vol. 116, pp. 91-102.

Flicker, S. and Guta, A. (2008) 'Ethical Approaches to Adolescent Participation in Sexual Health Research', Journal of Adolescent Health, Vol. 42, No. 1, pp. 3-10.

Flory, J. and Emanuel, E. (2004) 'Interventions to improve research participants' understanding in informed consent for research: A Systematic Review', Journal of the American Medical Association (JAMA), Vol. 292, No. 13, pp. 1593-1601.

Fontes, L.A. (2004) 'Ethics in Violence Against Women Research: The Sensitive, the Dangerous and the Overlooked', Ethics \& Behavior, Vol. 14, No. 2, pp. 141-74.

Ford, C., English, A. and Sigman, G. (2004) 'Confidential health care for adolescents: Position paper of the Society for Adolescent Medicine', Journal of Adolescent Health, Vol. 35, No. 2, pp. 160-67.

Ford, C.A., Thomsen, S.L. and Compton, B. (2001) 'Adolescents' interpretations of conditional confidentiality assurances', Journal of Adolescent Health, Vol. 29, No. 3, pp. 156-59.

Ford, K., Sankey, J. and Crisp, J. (2007) 'Development of children's assent documents using a child-centred approach', Journal of Child Health Care, Vol. 11, No. 1, pp. 19-28.

Fost, N. and Levine, R.J. (2007) 'The Dysregulation of Human Subjects Research', Journal of the American Medical Association (JAMA), Vol. 298, No. 18, pp. 2196-98.

Fox, J., Artemeva, N., Darville, R. and Woods, D. (2006) 'Juggling through Hoops: Implementing Ethics Policies in Applied Language Studies', Journal of Academic Ethics, Vol. 4, pp. 77-99.

Fox, R.M. (2005) 'Debate: Should Australia move towards a centralized ethics committees system? The case for', Internal Medicine Journal, Vol. 35, No. 4, pp. 247-48.

Frader, J.E. (2003) 'We need substantive criteria for decisions by children', The American Journal of Bioethics, Vol. 3, No. 4, pp. 8-9.

Fraser, H.E., Martlew, A.E. and Frew, D.J. (2007) 'Model for a single ethical and scientific review of multicentre research in New South Wales', Medical Journal of Australia, Vol. 187, No. 1, pp. 7-8.

Freeman, W.L. (1994) 'Research with Radiation and Healthy Children: Greater than Minimal Risk', IRB: Ethics and Human Research, Vol. 16, No. 5, pp. 1-5.

Friedman, L. and DeMets, D. (1981) 'The Data Monitoring Committee: How it operates and why', IRB: Ethics and Human Research, Vol. 3, No. 4, pp. 6-8.

Gandhi, R. (2005) 'Research involving Children: Regulations, Review Boards and Reform', Journal of Health Care Law \& Policy, Vol. 8, No. 2, pp. 264-330.

Garfield, P. (1995) 'Cross district comparison of applications to research ethics committees', British Medical Journal, Vol. 311, pp. 660-61.

Geller, G., Tambor, E.S., Bernhardt, B.A., Fraser, G. and Wissow, L.S. (2003) 'Informed consent for enrolling minors in genetic susceptibility research: A qualitative study of at-risk children's and parents' views about children's role in decisionmaking', Journal of Adolescent Health, Vol. 32, No. 4, pp. 260-71.

Glantz, L.H. (2002) 'Non-therapeutic Research with Children: Grimes v. Kennedy Krieger Institute', American Journal of Public Health, Vol. 92, No. 7, pp. 1070-73.

Glasby, J. and Beresford, P. (2007) 'In Whose Interests? Local Research Ethics Committees and Service User Research', Ethics and Social Welfare, Vol. 1, No. 3, pp. 282-92.

Goldner, J.A. (2000) 'Dealing with Conflicts of Interest in Biomedical Research: IRB Oversight as the Next Best Solution to the Abolitionist Approach', Journal of Law, Medicine \& Ethics, Vol. 28, No. 4, pp. 379-404.

Goodenough, T., Williamson, E., Kent, J. and Ashcroft, R. (2003) “"What did you think about that?" Researching Children's Perceptions of Participation in a Longitudinal Genetic Epidemiological Study', Children \& Society, Vol. 17, No. 2, pp. 113-25.

Gordon, B., Prentice, E. and Anderson, J. (2000) 'Conflict between Research Design and Minimization of Risks in Pediatric Research', IRB: Ethics and Human Research, Vol. 22, No. 3, pp. 1-6.

Gordon, B., Prentice, E. and Reitemeier, P. (1996) 'The Use of Normal Children as Participants in Research on Therapy', IRB: Ethics and Human Research, Vol. 18, No. 3, pp. 5-8.

Gordon, E.J. and Parsi, K.P. (2002) 'It's Alive! Giving Birth to Research Ethics Education', The American Journal of Bioethics, Vol. 2, No. 4, pp. 65-66.

Gordon, E.J., Yamokoski, A.H. and Kodish, E. (2006) 'Children, Research, and Guinea Pigs: Reflections on a Metaphor', IRB: Ethics \& Human Research, Vol. 28, No. 5, pp. 12-19.

Gordon, V.M., Sugarman, J. and Kass, N. (1998) 'Toward a more comprehensive approach to protecting human subjects: The Interface of Data Safety Monitoring Boards and Institutional Review Boards in Randomized Clinical Trials', IRB: Ethics and Human Research, Vol. 20, No. 1, pp. 1-5.

Grady, C. (2007) 'Quality Improvement and Ethical Oversight', Annals of Internal Medicine, Vol. 146, No. 9, pp. 680-81.

Grayson, J.P. and Myles, R. (2005) 'How research ethics boards are undermining survey research on Canadian university students', Journal of Academic Ethics, Vol. 2, No. 4, pp. 293-314. 
Green, L.W. and Mercer, S.L. (2001) 'Can Public Health Researchers and Agencies reconcile the push from funding bodies and the pull from communities?', American Journal of Public Health, Vol. 91, No. 12, pp. 1926-29.

Grinyer, A. (2002) 'The Anonymity of Research Participants: Assumptions, Ethics and Practicalities', Social Research Update, Vol. 36 (Spring).

Grover, S. (2003) 'On the Limits of Parental Proxy Consent: Children's right to non-participation in non-therapeutic research', Journal of Academic Ethics, Vol. 1, No. 4, pp. 349-83.

Grover, S. (2004) 'What's human rights got to do with it? On the proposed changes to SSHRC Ethics Research Policy', Journal of Academic Ethics, Vol. 2, No. 3, pp. 249-62.

Guillemin, M. and Gillam, L. (2004) 'Ethics, Reflexivity and "Ethically Important Moments" in Research', Qualitative Inquiry, Vol. 10, No. 2, pp. 261-80.

Gunsalus, C.K., Bruner, E.M., Burbules, N.C., Dash, L., Finkin, M., Goldberg, J.P., Greenough, W.T., Miller, G.A., Pratt, M.G., Iriye, M. and Aronson, D. (2007) 'The Illinois White Paper: Improving the System for Protecting Human Subjects: Counteracting IRB "Mission Creep"' ', Qualitative Inquiry, Vol. 13, No. 5, pp. 617-49.

Hadjistavropoulos, T. and Smythe, W.E. (2001) 'Elements of Risk in Qualitative Research', Ethics \& Behavior, Vol. 11, No. 2, pp. 163-74.

Haggerty, K.D. (2004) 'Ethics Creep: Governing Social Science Research in the name of Ethics', Qualitative Sociology, Vol. 27, No. 4, pp. 391-414.

Halila, R. and Lotjonen, S. (2003) 'Why shouldn't children decide whether they are enrolled in non-beneficial medical research?', The American Journal of Bioethics, Vol. 3, No. 4, pp. 35-36.

Halse, C. and Honey, A. (2007) 'Rethinking Ethics Review as Institutional Discourse', Qualitative Inquiry, Vol. 13, No. 3, pp. 336-52.

Hanna, K.E. (2000) 'Research Ethics: Reports, Scandal, Calls for Change', The Hastings Center Report, Vol. 30, No. 6, p. 6.

Harrison, J., MacGibbon, L. and Morton, M. (2001) 'Regimes of Trustworthiness in Qualitative Research: The Rigors of Reciprocity', Qualitative Inquiry, Vol. 7, No. 3, pp. 323-45.

Hauswald, D.D.M. (2002) 'Quality Improvement or Research: A Distinction without a Difference?', IRB: Ethics and Human Research, Vol. 24, No. 4, pp. 9-12.

Hearnshaw, H. (2004) 'Comparison of requirements of research ethics committees in 11 European countries for a noninvasive interventional study', British Medical Journal, Vol. 328, No. 7432, pp. 140-41.

Heath, E.J. (1979) 'The IRB's Monitoring Function: Four Concepts of Monitoring', IRB: Ethics and Human Research, Vol. 1, No. 5, pp. 1-12.

Heath, S., Charles, V., Crow, G. and Wiles, R. (2007) 'Informed consent, gatekeepers and go-betweens: Negotiating consent in child- and youth-orientated institutions', British Educational Research Journal, Vol. 33, No. 3, pp. 403-17.

Hedgecoe, A., Carvalho, F., Lobmayer, P. and Raka, F. (2006) 'Research ethics committees in Europe: Implementing the directive, respecting diversity', Journal of Medical Ethics, Vol. 32, No. 8, pp. 483-86.

Helgeland, I.M. (2005) " “Catch 22" of Research Ethics: Ethical dilemmas in follow-up studies of marginal groups', Qualitative Inquiry, Vol. 11, No. 4, pp. 549-69.

Hemmings, A. (2006) 'Great Ethical Divides: Bridging the gap between institutional review boards and researchers', Educational Researcher, Vol. 35, No. 4, pp. 12-18.

Herrera, C. (2000) 'Research Ethics and the "Fieldwork Monitoring Committee" ', IRB: Ethics \& Human Research, Vol. 22, No. 6, pp. 11-13.

Hesse-Biber, S.N. and Leavy, P. (2006) 'The Ethics of Social Research'. In: The Practice of Qualitative Research. Thousand Oaks, CA: Sage, pp. 83-116.

Hewison, J. and Haines, A. (2006) 'Overcoming barriers to recruitment in health research', British Medical Journal, Vol. 333, No. 7562, pp. 300-02.

HHS (2005) Code of Federal Regulations. Title 45 - Public Welfare: Department of Health and Human Services, Part 46: Protection of Human Subjects. Washington, DC: US Department of Health and Human Services. Available at: www.hhs. gov/ohrp/humansubjects/guidance/45cfr46.htm (accessed February 2010)

Hirtle, M., Lemmens, T. and Sprumont, D. (2000) 'A Comparative Analysis of Research Ethics Review Mechanisms and the ICH Good Clinical Practice Guideline', European Journal of Health Law, Vol. 7, No. 3, pp. 265-92.

Hochhauser, M. (1999) 'Informed Consent and Patient' Rights Documents: A Right, a Rite or a Rewrite?', Ethics \& Behavior, Vol. 9, No. 1, pp. 1-20.

Hochhauser, M. (2002) ' "Therapeutic Misconception" and "Recruiting Doublespeak" in the Informed Consent Process', IRB: Ethics and Human Research, Vol. 24, No. 1, pp. 11-12.

Hofmann, A.D. (1980) 'A rational policy toward consent and confidentiality in adolescent health care', Journal of Adolescent Health Care, Vol. 1, No. 1, pp. 9-17.

Holaday, B., Gonzales, 0. and Mills, D. (2007) 'Assent of School-age Bilingual Children', Western Journal of Nursing Research, Vol. 29, No. 4, pp. 466-85.

Holland, K. (2007) 'The Epistemological Bias of Ethics Review: Constraining Mental Health Research', Qualitative Inquiry, Vol. 13, No. 6, pp. 895-913.

Holm, S. (1992) 'How many lay members can you have in your IRB?: An Overview of the Danish System', IRB: Ethics and Human Research, Vol. 14, No. 6, pp. 8-11.

Holt, L. (2004) 'The "voices" of children: De-centring empowering research relations', Children's Geographies, Vol. 21, No. 1, pp. $13-27$.

Homan, R. (2001) 'The Principle of Assumed Consent: The Ethics of Gatekeeping', Journal of Philosophy of Education, Vol. 35, No. 3, pp. 329-43.

Hood, S., Kelley, P. and Mayall, B. (1996) 'Children as Research Subjects: A Risky Enterprise', Children \& Society, Vol. 10, No. 2, pp. 117-28. 
Hoop, J.G., Smyth, A.C. and Roberts, L.W. (2008) 'Ethical Issues in Psychiatric Research on Children and Adolescents', Child and Adolescent Psychiatric Clinics of North America, Vol. 17, No. 1, pp. 127-48.

Hopkins, P.E. (2007) 'Positionalities and Knowledge: Negotiating Ethics in Practice', ACME: An International E-Journal for Critical Geographies, Vol. 6, No. 3, pp. 386-94.

Horton, J. (2001) ' “Do you get some funny looks when you tell people what you do?" Muddling through some angsts and ethics of (being a male) researching with children', Ethics, Place \& Environment, Vol. 4, No. 2, pp. 159-66.

Howarth, M.L. and Kneafsey, R. (2005) 'The impact of research governance in healthcare and higher education organizations', Journal of Advanced Nursing, Vol. 49, No. 6, pp. 675-83.

Howe, K.R. and Dougherty, K.C. (1993) 'Ethics, institutional review boards and the changing face of educational research', Educational Researcher, Vol. 22, No. 9, pp. 16-21.

Howe, K.R. and Moses, M.S. (1999) 'Ethics in Educational Research', Review of Research in Education, Vol. 24, No. 1, pp. 21-59.

Howitt, R. (2005) 'Human Ethics, Supervision and Equity: Ethical Oversight of Student Research', Journal of Geography in Higher Education, Vol. 29, No. 3, pp. 317-20.

HRC (1996) HRC Guidelines for Ethics Committee Accreditation. Wellington, New Zealand: Health Research Council.

HSE Research Ethics Committee Review Group (2008) Review of Research Ethics Committees and Processes in Republic of Ireland. Available at: www.hse.ie/eng/services/Publications/corporate/etr/Review_of_Research_Ethics.pdf (accessed February 2010)

Huber, J. and Clandinin, D.J. (2002) 'Ethical dilemmas in relational narrative inquiry with children', Qualitative Inquiry, Vol. 8, No. 6, pp. 785-803.

Hunter, D. (2007a) 'Efficiency and the proposed reforms to the NHS research ethics system', Journal of Medical Ethics, Vol. 33, No. 11, pp. 651-54.

Hunter, D. (2007b) 'Proportional ethical review and the identification of ethical issues', Journal of Medical Ethics, Vol. 33 , No. 4, pp. 241-45.

Hunter, D. and Pierscionek, B.K. (2007) 'Children, Gillick competency and consent for involvement in research', Journal of Medical Ethics, Vol. 33, No. 11, pp. 659-62.

ICB (2004) Operational Procedures for Research Ethics Committees: Guidance 2004. Dublin: Irish Council for Bioethics.

ICRIN (2008) Recommendations of Working Group 1: Ethics and Interaction with Ethics Committees. Dublin: Irish Clinical Research Infrastructure Network, Molecular Medicine Ireland.

Jackson, S. and Hafemeister, T.L. (2001) 'Impact of parental consent and notification policies on the decisions of adolescents to be tested for HIV', Journal of Adolescent Health, Vol. 29, No. 2, pp. 81-93.

Jacobson, N., Gewurtz, R. and Haydon, E. (2007) 'Ethical Review of Interpretive Research: Problems and Solutions', IRB: Ethics \& Human Research, Vol. 29, No. 5, pp. 1-8.

Janovicek, N. (2006) 'Oral History and Ethical Practice: Towards Effective Policies and Procedures', Journal of Academic Ethics, Vol. 4, pp. 157-74.

Jansen, L.A. (2006) 'The Problem with Optimism in Clinical Trials', IRB: Ethics \& Human Research, Vol. 28, No. 4, pp. 13-19.

Joffe, S. (2003) 'Rethink "Affirmative Agreement," but abandon "Assent"', The American Journal of Bioethics, Vol. 3, No. 4, pp. 9-11.

Joffe, S., Cook, E.F., Cleary, P.D., Clark, J.W. and Weeks, J.C. (2001) 'Quality of informed consent in cancer clinical trials: A cross-sectional survey', The Lancet, Vol. 358, No. 9295, pp. 1772-77.

John, J.E. (2007) 'The child's right to participate in research: Myth or misconception?', British Journal of Nursing, Vol. 16, No. 3, pp. 157-60.

Katz, J. (1995) 'Do we need another advisory commission on human experimentation?', The Hastings Center Report, Vol. 25, No. 1, pp. 29-31.

Kelley, P., Mayall, B. and Hood, S. (1997) 'Children's Accounts of Risk', Childhood, Vol. 4, No. 3, pp. 305-24.

Kelley, P.C., Agle, B.R. and DeMott, J. (2005) 'Mapping our progress: Identifying, categorizing and comparing universities' ethics infrastructures', Journal of Academic Ethics, Vol. 3, No. 2-4, pp. 205-29.

Kennedy, J.E. (2006) 'Grey Matter: Ambiguities and Complexities of Ethics in Research', Journal of Academic Ethics, Vol. 3, No. 2-4, pp. 143-58.

Kerrison, S. and Pollock, A.M. (2005) 'The reform of UK research ethics committees: Throwing the baby out with the bath water?', Journal of Medical Ethics, Vol. 31, No. 8, pp. 487-89.

Kessel, R. (1993) 'In the UK, children can't just say no', The Hastings Center Report, Vol. 23, No. 2, p. 20.

Khanlou, N. and Peter, E. (2005) 'Participatory action research: Considerations for ethical review', Social Science \& Medicine, Vol. 60, No. 10, pp. 2333-40.

Kimmelman, J. (2007) 'The Therapeutic Misconception at 25', The Hastings Center Report, Vol. 37, No. 6, pp. 36-42.

King, N.M.P. (2000) ‘Defining and describing benefit appropriately in clinical trials', Journal of Law, Medicine \& Ethics, Vol. 28, No. 4, pp. 332-43.

King, N.M.P., Henderson, G.E., Churchill, L.R., Davis, A.M., Hull, S.C., Nelson, D.K., Parham-Vetter, P.C., Rothschild, B.B., Easter, M.M. and Wilfond, B.S. (2005) 'Consent Forms and the Therapeutic Misconception: The Example of Gene Transfer Research', IRB: Ethics \& Human Research, Vol. 27, No. 1, pp. 1-8.

Kitchin, H. (2003) 'The Tri-Council Policy Statement and Research in Cyberspace: Research Ethics, the Internet and revising a "Living Document" ', Journal of Academic Ethics, Vol. 1, No. 4, pp. 397-418.

Kodish, E. (2003) 'Pediatric ethics and early-phase childhood cancer research: Conflicted goals and the prospect of benefit', Accountability in Research, Vol. 10, No. 1, pp. 17-25.

Komesaroff, P. (2005) 'Debate: Should Australia move towards a centralized ethics committee system? The case against', Internal Medicine Journal, Vol. 35, No. 4, pp. 249-50.

Kon, A.A. (2007) 'The Risky Business of assessing Research Risk', American Journal of Bioethics, Vol. 7, No. 3, pp. $21-22$. 
Kon, A.A. and Klug, M. (2006) 'Methods and practices of investigators for determining participants' decisional capacity and comprehension of protocols', Journal of Empirical Research on Human Research Ethics, Vol. 1, No. 4, pp. 61-68.

Kopelman, L.M. (2000) 'Children as Research Subjects: A Dilemma', Journal of Medicine \& Philosophy, Vol. 25, No. 6, pp. 745-64.

Kopelman, L.M. (2002) 'Group Benefit and Protection of Pediatric Research Subjects: Grimes v. Kennedy Krieger and the Lead Abatement Study', Accountability in Research, Vol. 9, No. 3, pp. 177-92.

Ladd, R.E. (2003) 'Child Assent Revisited', The American Journal of Bioethics, Vol. 3, No. 4, pp. 37-38.

Lanphear, B.P., Paulson, J. and Beirne, S. (2006) 'Trials and tribulations of protecting children from environmental hazards', Environmental Health Perspectives, Vol. 114, No. 10, pp. 1609-12.

Lantos, J. D. (2007) 'Research in Wonderland: Does "Minimal Risk" mean whatever an institutional review board says it means?', American Journal of Bioethics, Vol. 7, No. 3, pp. 11-12.

Larson, E., Bratts, T., Zwanziger, J. and Stone, P. (2004) 'A survey of IRB process in 68 US hospitals', Journal of Nursing Scholarship, Vol. 36, No. 3, pp. 260-64.

Lawson, S.L. and Adamson, H.M. (1995) 'Informed Consent Readability: Subject understanding of 15 common consent form phrases', IRB: Ethics and Human Research, Vol. 17, No. 5-6, pp. 16-19.

Lederer, S. and Grodin, M. (1994) 'Historical Overview: Pediatric Experimentation'. In: S. Lederer and M. Grodin (eds.), Children as research subjects: Science, ethics and law. New York: 0xford University Press, pp. 3-27.

Lederer, S.E. (2003) 'Children as Guinea Pigs: Historical Perspectives', Accountability in Research, Vol. 10, No. 1, pp. 1-16.

Lehrer, J.A., Pantell, R., Tebb, K. and Shafer, M.-A. (2007) 'Forgone Health Care among US Adolescents: Associations between risk characteristics and confidentiality concern', Journal of Adolescent Health, Vol. 40, No. 3, pp. 218-26.

Levine, R. (1995) 'Adolescents as research subjects without permission of their parents or guardians: Ethical Considerations', Journal of Adolescent Health, Vol. 17, No. 5, pp. 287-97.

Lincoln, Y.S. and Tierney, W.G. (2004) 'Qualitative Research and Institutional Review Boards', Qualitative Inquiry, Vol. 10, No. 2, pp. 219-34.

Liss, M.B. (1994) 'Child Abuse: Is there a mandate for researchers to report?', Ethics \& Behavior, Vol. 4, No. 2, pp. $133-46$.

Loff, B. and Black, J. (2004) 'Research Ethics Committees: What is their contribution?', Medical Journal of Australia, Vol. 181, No. 8 , pp. 440-41.

Lothen-Kline, C., Howard, D.E., Hamburger, E.K., Worrell, K.D. and Boekeloo, B.0. (2003) 'Truth and consequences: Ethics, confidentiality and disclosure in adolescent longitudinal prevention research', Journal of Adolescent Health, Vol. 33, No. 5, pp. 385-94.

Lynn, J. (2004) 'When does quality improvement count as research? Human subject protection and theories of knowledge', Quality \& Safety in Health Care, Vol. 13, No. 1, pp. 67-70.

Lyren, A., Kodish, E. and Lazebnik, R. (2004) 'Understanding confidentiality in the adolescent patient/doctor relationship: A pilot study (Phase 1)', Journal of Adolescent Health, Vol. 34, No. 2, pp. 114-15.

Lyren, A., Kodish, E., Lazebnik, R. and 0’Riordan, M.A. (2006) 'Understanding Confidentiality: Perspectives of African American adolescents and their parents', Journal of Adolescent Health, Vol. 39, No. 2, pp. 261-65.

Macaulay, A.C., Ing, A., Salsberg, J., McGregor, A., Saad-Haddad, C., Rice, J., Montour, L. and Gray-Donald, K. (2007) 'Community-based Participatory Research: Lessons from sharing results with the community, Kahnawake Schools Diabetes Prevention Project', Progress in Community Health Partnerships: Research, Education, and Action, Vol. 1, No. 2, pp. 143-52.

MacNaughton, G. and Smith, K. (2005) 'Transforming research ethics: The choices and challenges of researching with children'. In: A. Farrell (ed.), Ethical Research with Children. New York: Open University Press, pp. 112-23.

Malone, S. (2003) 'Ethics at home: Informed consent in your own backyard', International Journal of Qualitative Studies in Education, Vol. 16, No. 6, pp. 797-815.

Mammel, K.A. and Kaplan, D.W. (1995) 'Research Consent by Adolescent Minors and Institutional Review Boards', Journal of Adolescent Health, Vol. 17, No. 5, pp. 323-30.

Martin, D.G. (2007) 'Bureaucratizing Ethics: Institutional Review Boards and Participatory Research', ACME: An International E-Journal for Critical Geographies, Vol. 6, No. 3, pp. 319-28.

Martin, M. and Lantos, J. (2005) 'Bioethics meets the Barrio: Community-based Research involving Children' In: E. Kodish (ed.), Ethics and Research with Children: A Case-based Approach. New York: Oxford University Press, pp. 63-76.

Martin, R.A. and Robert, J.S. (2007) 'Is risky pediatric research without prospect of direct benefit ever justified?', American Journal of Bioethics, Vol. 7, No. 3, pp. 12-15.

Marzano, M. (2007) 'Informed Consent, Deception and Research Freedom in Qualitative Research', Qualitative Inquiry, Vol. 13, No. 3, pp. 417-36.

Mastroianni, A. and Kahn, J. (2001) 'Swinging on the Pendulum', The Hastings Center Report, Vol. 31, No. 3, pp. 21-28.

Mastroianni, A.C. and Kahn, J.P. (2002) 'Risk and Responsibility: Ethics, Grimes v. Kennedy Krieger, and Public Health Research involving Children', American Journal of Public Health, Vol. 92, No. 7, pp. 1073-76.

Matsui, K., Lie, R.K. and Kita, Y. (2007) 'Two methods of obtaining informed consent in a genetic epidemiological study: Effects on understanding', Journal of Empirical Research on Human Research Ethics, Vol. 2, No. 3, pp. 39-48.

Mayeux, L., Underwood, M.K. and Risser, S.D. (2007) 'Perspectives on the Ethics of Sociometric Research with Children: How children, peers, and teachers help to inform the debate', Merrill-Palmer Quarterly, Vol. 53, No. 1, pp. 53-78.

McCabe, M.A. (1996) 'Involving children and adolescents in medical decision-making: Developmental and Clinical Considerations', Journal of Pediatric Psychology, Vol. 21, No. 4, pp. 505-16.

McCabe, M.A., Rushton, C.H., Glover, J., Murray, M.G. and Leikin, S. (1996) 'Implications of the patient self-determination act: Guidelines for involving adolescents in medical decision-making', Journal of Adolescent Health, Vol. 19, No. 5, pp. 319-24. 
McCarthy, D.B., Shatin, D., Drinkard, C.R., Kleinman, J.H. and Gardner, J.S. (1999) 'Medical records and privacy: Empirical effects of legislation', Health Services Research, Vol. 34, No. 1, Part 2, pp. 417-25.

McDowell, L. (2001) "It's that Linda again": Ethical, practical and political issues involved in longitudinal research with young men', Ethics, Place \& Environment, Vol. 4, No. 2, pp. 87-100.

McGee, E.M. (2003) 'Altruism, Children and Non-beneficial Research', The American Journal of Bioethics, Vol. 3, No. 4, pp. 21-23.

McKechnie, L. (2006) 'Observations of Babies and Toddlers in Library Settings', Library Trends, Vol. 55, No. 1, pp. $190-201$.

McLaren, H. (2007) 'Exploring the Ethics of Forewarning: Social Workers, Confidentiality and Potential Child Abuse Disclosures', Ethics and Social Welfare, Vol. 1, No. 1, pp. 22-40.

McLaughlin, H. (2005) 'Young Service Users as Co-researchers: Methodological Problems and Possibilities', Qualitative Social Work, Vol. 4, No. 2, pp. 211-28.

McLaughlin, H. (2007) 'Ethical Issues in the Involvement of Young Service Users in Research', Ethics and Social Welfare, Vol. 1, No. 2, pp. 176-93.

McNeill, P.M. (1989) 'Research Ethics Review in Australia, Europe and North America', IRB: Ethics and Human Research, Vol. 11, No. 3, pp. 4-7.

McNeill, P.M. (2001) 'A Critical Analysis of Australian Clinical Ethics Committees and the functions they serve', Bioethics, Vol. 15, No. 5-6, pp. 443-60.

McWilliams, R., Hebden, C.W. and Gilpin, A.M.K. (2006) 'Concept Paper: A Virtual Centralized IRB System', Accountability in Research, Vol. 13, No. 1, pp. 25-45.

McWilliams, R., Hoover-Fong, J., Hamosh, A., Beck, S., Beaty, T. and Cutting, G. (2003) 'Problematic Variation in Local Institutional Review of a Multicenter Genetic Epidemiology Study', Journal of the American Medical Association (JAMA), Vol. 290, No. 3, pp. 360-66.

Meade, C.D. and Howser, D.M. (1992) 'Consent Forms: How to determine and improve their readability', Oncology Nursing Forum, Vol. 19, No. 10, pp. 1523-28.

Menasche, A. (1995) 'Pending legislation threatens adolescent research', IRB: Ethics and Human Research, Vol. 17, No. 4, pp. $9-10$.

Merlo, D.F., Knudsen, L.E., Matusiewicz, K., Niebroj, L. and Vahakangas, K.H. (2007) 'Ethics in studies on children and environmental health', Journal of Medical Ethics, Vol. 33, No. 7, pp. 408-13.

Meslin, E.M. (2006) 'Shifting Paradigms in Health Services Research Ethics. Consent, Privacy and the Challenges for IRBs', Journal of General Internal Medicine, Vol. 21, No. 3, pp. 279-80.

Miller, F.G. and Brody, H. (2003) 'A Critique of Clinical Equipoise: Therapeutic Misconception in the Ethics of Clinical Trials', The Hastings Center Report, Vol. 33, No. 3, pp. 19-28.

Miller, F.G. and Joffe, S. (2006) 'Evaluating the Therapeutic Misconception', Kennedy Institute of Ethics Journal, Vol. 16, No. 4, pp. 353-66.

Millstein, S.G. and Halpern-Felsher, B.L. (2002) 'Perceptions of risk and vulnerability', Journal of Adolescent Health, Vol. 31, No. 1 (Supplement 1), pp. 10-27.

Minkler, M., Fadem, P., Perry, M., Blum, K., Moore, L. and Rogers, J. (2002) 'Ethical Dilemmas in Participatory Action Research: A Case Study from the Disability Community', Health Education and Behavior, Vol. 29, No. 1, pp. 14-29.

Mishna, F., Antle, B.J. and Regehr, C. (2004) 'Tapping the Perspectives of Children: Emerging ethical issues in qualitative research', Qualitative Social Work, Vol. 3, No. 4, pp. 449-68.

Moerman, C.J., Haafkens, J.A., Söderström, M., Rásky, É., Maguire, P., Maschewsky-Schneider, U., Norstedt, M., Hahn, D., Reinerth, H. and McKevitt, N. (2007) 'Gender equality in the work of local research ethics committees in Europe: A study of practice in five countries', Journal of Medical Ethics, Vol. 33, No. 2, pp. 107-12.

Mold, J.W. and Peterson, K.A. (2005) 'Primary Care Practice-based Research Networks: Working at the interface between research and quality improvement', Annals of Family Medicine, Vol. 3, Supplement 1, pp. S12-20.

Moolchan, E.T. and Mermelstein, R. (2002) ,Research on tobacco use among teenagers: Ethical challenges', Journal of Adolescent Health, Vol. 30, No. 6, pp. 409-17.

Moreno, J. (1995) Deciding Together: Bioethics and Moral Consensus. New York: Oxford University Press.

Moreno, J.D. (2001) 'Goodbye to All That', The Hastings Center Report, Vol. 31, No. 3, pp. 9-17.

Morrow, V. (2005) 'Ethical issues in collaborative research with children'. In: A. Farrell (ed.), Ethical Research with Children. New York: Open University Press, pp. 150-65.

Morrow, V. and Richards, M. (1996) 'The Ethics of Social Research with Children: An Overview', Children \& Society, Vol. 10, No. 2, pp. 90-105.

Murphy, T.F. (2003) 'Assent and Dissent in 407 Research with Children', The American Journal of Bioethics, Vol. 3, No. 4, pp. $18-19$.

Myser, C. (2004) 'Community-based Participatory Research in United States Bioethics: Steps toward more democratic theory and policy', The American Journal of Bioethics, Vol. 4, No. 2, pp. 67-68.

National Advisory Committee on Health and Disability Support Services Ethics (2003) Review of the Current Processes for Ethical Review of Health and Disability Research in New Zealand: Report to the Minister of Health. Wellington, New Zealand: National Advisory Committee on Health and Disability Support Services Ethics, Ministry of Health.

National Institute of Health, Office for Human Research Protections, Association of American Colleges and American Society of Clinical Oncology (2005) Alternative Models of IRB Review: Workshop Summary Report. Washington, DC: US Department of Health and Human Services.

NEAC (2003) Ethical Review of Observational Research, Audit and Related Activities: Discussion Document, National Advisory Committee on Health and Disability Support Services Ethics. Wellington, New Zealand: National Ethics Advisory Committee, Ministry of Health. 
Neill, S.J. (2005) 'Research with children: A critical review of the guidelines', Journal of Child Health Care, Vol. 9, No. 1, pp. 46-58.

Nelson, C. (2004) 'The Brave New World of Research Surveillance', Qualitative Inquiry, Vol. 10, No. 2, pp. $207-18$.

Nelson, R.M. and Reynolds, W.W. (2003) 'We should reject passive resignation in favor of requiring the assent of younger children for participation in non-beneficial research', The American Journal of Bioethics, Vol. 3, No. 4, pp. 11-13.

Ness, R.B. (2007) 'Influence of the HIPAA Privacy Rule on Health Research' [for the Joint Policy Committee, Societies of Epidemiology], Journal of the American Medical Association (JAMA), Vol. 298, No. 18, pp. 2164-70.

NHMRC (2003a) Values and Ethics: Guidelines for Ethical Conduct in Aboriginal and Torres Strait Islander Health Research. Canberra, Australia: National Health and Medical Research Council.

NHMRC (2003b) When does quality assurance in health care require independent ethical review? Advice to Institutions, Human Research Ethics Committees and Health Care Professionals. Canberra, Australia: National Health and Medical Research Council.

NHMRC (2007) National statement on ethical conduct in research involving humans. Canberra, Australia: National Health and Medical Research Council.

Nilsen, R.D. and Rogers, B. (2005) “"That's not a good idea, mom": Negotiating children's subjectivity while constructing "home" as a research site', Children's Geographies, Vol. 3, No. 3, pp. 345-62.

NPSA (2007) Information Sheets and Consent Forms. Guidance for Researchers and Reviewers (Version 3.2). London: National Patient Safety Agency and National Research Ethics Service.

NRES (2007) Standard Operating Procedures for Research Ethics Committees in the United Kingdom ('SOPs') (Version 3.4). London: National Research Ethics Service.

NZHDEC (2006) Operational Standard for Ethics Committees, New Zealand Health and Disability Ethics Committees. Wellington, New Zealand: Ministry of Health.

O'Beirne, M., Stingl, M. and Hayward, S. (2007) 'Who reviews the projects of unaffiliated researchers for ethics? A Case Study from Alberta', Cambridge Quarterly of Healthcare Ethics, Vol. 16, No. 3, pp. 346-55.

O’Donnell, L.N., Duran, R.H., San Doval, A., Breslin, M.J., Juhn, G.M. and Stueve, A. (1997) 'Obtaining written parent permission for school-based health surveys of urban young adolescents', Journal of Adolescent Health, Vol. 21, No. 6, pp. 376-83.

Office of Inspector General (1998) Final Report on Low-Volume Institutional Research Boards, Memo Report No. 0EI-01-9700194. Washington, DC: US Department of Health and Human Services.

OHRP, NIH, AAMC and ASCO (2006) National Conference on Alternative IRB Models: Optimizing Human Subjects Protection, 19-21 November 2006, Washington, DC.

OMCYA (1999/2009) Children First: National Guidelines for the Protection and Welfare of Children, Office of the Minister for Children and Youth Affairs, Department of Health and Children (revised edition 2009). Dublin: The Stationery Office.

Ondrusek, N., Abramovitch, R., Pencharz, P. and Koren, G. (1998) 'Empirical examination of the ability of children to consent to clinical research', Journal of Medical Ethics, Vol. 24, No. 3, pp. 158-65.

OSTP (1991) 'Part II: Federal Policy for the Protection of Human Subjects: Notices and Rules (Office of Science and Technology Policy)', Federal Register, Vol. 56, No. 117, pp. 28002-12.

Owen, M. (2006) 'Conflict and Convergence: The Ethics Review of Action Research', Journal of Academic Ethics, Vol. 4, pp. 61-75.

Owen, S. (2001) 'The practical, methodological and ethical dilemmas of conducting focus groups with vulnerable clients', Journal of Advanced Nursing, Vol. 36, No. 5, pp. 652-58.

Paasche-Orlow, M.K., Taylor, H.A. and Brancati, F.L. (2003) 'Readability Standards for Informed Consent Forms as compared with Actual Readability', The New England Journal of Medicine, Vol. 348, No. 8, pp. 721-26.

Palca, J. (1996) 'Institutional Review Boards: A net too thin', The Hastings Center Report, Vol. 26, No. 3, p. 4.

Pasternak, R.H., Cheng, T.L., Geller, G. and Parrish, C. (2005) 'Parent and adolescent views of risk behavior research and consent', Journal of Adolescent Health, Vol. 36, No. 2, pp. 104-05.

Patterson, D. (2008) 'Research Ethics Boards as Spaces of Marginalization: A Canadian Story', Qualitative Inquiry, Vol. 14, No. 1 , pp. 18-27.

Paulson, J.A. (2006) 'An Exploration of Ethical Issues in Research in Children's Health and the Environment', Environmental Health Perspectives, Vol. 114, No. 10, pp. 1603-08.

Pentz, R.D. and Khayat, A.F. (2004) 'The Poster Child for the Need for Central Review of Research Protocols: The Children's Oncology Group', Cambridge Quarterly of Healthcare Ethics, Vol. 13, No. 4, pp. 359-65.

Perneger, T.V. (2004) 'Why we need ethical oversight of quality improvement projects', International Journal for Quality in Health Care, Vol. 16, No. 5, pp. 343-44.

Piercy, H. and Hargate, M. (2004) 'Social research on the under-16s: A consideration of the issues from a UK perspective', Journal of Child Health Care, Vol. 8, No. 4, pp. 253-63.

Pinkerton, C.R., Ablett, S., Boos, J. and Philip, T. (2002) 'Ethical approval for multicentre clinical trials in children: Contrasting systems in three European countries', European Journal of Cancer, Vol. 38, No. 8, pp. 1051-58.

Powell, M.A. and Smith, A.B. (2006) 'Ethical guidelines for research with children: A review of current research ethics documentation in New Zealand', Kōtuitui: New Zealand Journal of Social Sciences Online, Vol. 1, No. 2, pp. $125-38$.

PRE (2004) Giving Voice to the Spectrum: Report of the Social Sciences and Humanities Research Ethics Special Working Committee to the Interagency Advisory Panel on Research Ethics. Ottawa, Canada: Interagency Advisory Panel on Research Ethics.

PRE (2007) REB Community Member Educational Needs: A PRE Pilot Project (by Interagency Advisory Panel Standing Committee on Education). Ottawa, Canada: Interagency Advisory Panel and Secretariat on Research Ethics.

Prentice, K.J., Appelbaum, P.S., Conley, R.R. and Carpenter, W.T. (2007) 'Maintaining informed consent validity during lengthy research protocols', IRB: Ethics \& Human Research, Vol. 29, No. 6, pp. 1-6. 
Prideaux, D. and Rogers, W. (2006) 'Audit or research: The ethics of publication', Medical Education, Vol. 40, No. 6, pp. 497-99.

Prilleltensky, I., Rossiter, A. and Walsh-Bowers, R. (1996) 'Toward a participatory framework for applied ethics. Preventing harm and promoting ethical discourse in the helping professions: Conceptual, research, analytical and action frameworks', Ethics \& Behavior, Vol. 6, No. 4, pp. 287-306.

Punch, S. (2002) 'Research with Children: The same or different from research with adults?', Childhood, Vol. 9, No. 3, pp. 321-41.

Putney, S.B. and Gruskin, S. (2002) 'Time, Place and Consciousness: Three dimensions of meaning for US Institutional Review Boards', American Journal of Public Health, Vol. 92, No. 7, pp. 1067-70.

Quadrel, M.J., Fischhoff, B. and Davis, W. (1993) 'Adolescent (In)vulnerability', American Psychologist, Vol. 48, No. 2, pp. 102-16.

Rambo, C. (2007) 'Handing IRB an Unloaded Gun', Qualitative Inquiry, Vol. 13, No. 3, pp. 353-67.

Ramcharan, P. and Cutcliffe, J.R. (2001) 'Judging the ethics of qualitative research: Considering the "ethics as process" model', Health \& Social Care in the Community, Vol. 9, No. 6, pp. 358-66.

Range, L.M. and Cotton, C.R. (1995a) 'Assent and Permission Rejoinder', Ethics \& Behavior, Vol. 5, No. 4, pp. 345-47.

Range, L.M. and Cotton, C.R. (1995b) 'Reports of Assent and Permission in Research With Children: Illustrations and Suggestions', Ethics \& Behavior, Vol. 5, No. 1, pp. 49-66.

Resnik, D.B. (2000) 'Financial Interests and Research Bias', Perspectives on Science, Vol. 8, No. 3, pp. 255-85.

Resnik, D.B. (2004) 'Liability for Institutional Review Boards', Journal of Legal Medicine, Vol. 25, No. 2, pp. 131-84.

Resnik, D.B. (2007) 'The New EPA Regulations for Protecting Human Subjects: Haste makes Waste', The Hastings Center Report, Vol. 37, No. 1, pp. 17-21.

Resnick, M.D., Bearinger, L.H., Sieving, R.E. and Eisenberg, M. (2003) 'Parental perspectives on restricting adolescents' reproductive health options: A population-based survey of parents of teens', Journal of Adolescent Health, Vol. 32, No. 2, p. 133.

Reynolds, W.W. and Nelson, R.M. (2007) 'Risk perception and decision processes underlying informed consent to research participation', Social Science \& Medicine, Vol. 65, No. 10, pp. 2105-15.

Reynolds, W.W. and Nelson, R.M. (2008) 'Empirical Data and the Acceptability of Research Risk: A Commentary on the Charitable Participation Standard', Archives of Pediatrics \& Adolescent Medicine, Vol. 162, No. 1, pp. 88-90.

Rhodes, R. (2002) 'The Pressing Need for Postdoctoral Research Ethics Education', The American Journal of Bioethics, Vol. 2, No. 4.

Richardson, S. and McMullan, M. (2007) 'Research Ethics in the UK: What can sociology learn from health?', Sociology, Vol. 41, No. 6, pp. 1115-32.

Ripley, E.B.D., Macrina, F.L. and Markowitz, M. (2006) 'Paying Clinical Research Participants: One Institution's Research Ethics Committee's Perspective', Journal of Empirical Research on Human Research Ethics, Vol. 1, No. 4, pp. 37-44.

Risjord, M. and Greenberg, J. (2002) 'When IRBs Disagree: Waiving Parental Consent for Sexual Health Research on Adolescents', IRB: Ethics and Human Research, Vol. 24, No. 2, pp. 8-14.

Rivera, R., Borasky, D., Carayon, F., Rice, R., Kirkendale, S., Wilson, W. L. and Woodsong, C. (2004) Research Ethics Training Curriculum for Community Representatives. Research Triangle Park, NC: Family Health International.

Roberts, L.M., Bowyer, L., Homer, C.S. and Brown, M.A. (2004) 'Multicentre research: Negotiating the ethics approval obstacle course', Medical Journal of Australia, Vol. 180, No. 3, p. 139.

Roberts, L.W. and McAuliffe, T.L. (2006) 'Investigators' affirmation of ethical, safeguard and scientific commitments in human research', Ethics \& Behavior, Vol. 16, No. 2, pp. 135-50.

Roberts, L.W., Warner, T.D. and Hammond, K.A.G. (2005) 'Coexisting Commitments to Ethics and Human Research: A preliminary study of the perspectives of 83 medical students', The American Journal of Bioethics, Vol. 5, No. 6, pp. 1-7.

Roberts, M.C. and Buckloh, L.M. (1995) 'Five Points and a Lament about Range and Cotton's Reports of Assent and Permission in Research With Children: Illustrations and Suggestions', Ethics \& Behavior, Vol. 5, No. 4, pp. 333-44.

Robinson, W.M. (2003) 'What's altruism got to do with it?', The American Journal of Bioethics, Vol. 3, No. 4, pp. $23-24$.

Robson, C. (2002) Real World Research (2nd edition). Malden, MA: Blackwell Publishing.

Rogers, A.S., D’Angelo, L. and Futterman, D. (1994) 'Guidelines for Adolescent Participation in Research: Current Realities and Possible Resolutions', IRB: Ethics and Human Research, Vol. 16, No. 4, pp. 1-6.

Rogers, A.S., Schwartz, D.F., Weissman, G. and English, A. (1999) 'A Case Study in Adolescent Participation in Clinical Research: Eleven Clinical Sites, One Common Protocol and Eleven IRBs', IRB: Ethics and Human Research, Vol. 21, No. 1, pp. 6-10.

Rojas, N.L., Sherrit, L., Harris, S. and Knight, J.R. (2008) 'The Role of Parental Consent in Adolescent Substance Use Research', Journal of Adolescent Health, Vol. 42, No. 2, pp. 192-97.

Rosato, J. (2000) 'The ethics of clinical trials: A child's view', Journal of Law, Medicine \& Ethics, Vol. 28, No. 4, pp. 362-78.

Roseman, K.D. (1987) 'Man-Child in a Promised Land: A Layman serves on the Human Subjects Committee', IRB: Ethics and Human Research, Vol. 9, No. 2, pp. 8-10.

Ross, L.F. (2004a) 'Children in Medical Research. Balancing protection and access: Has the pendulum swung too far?', Perspectives in Biology and Medicine, Vol. 47, No. 4, pp. 519-36.

Ross, L.F. (2004b) 'Convening a 407 Panel for research not otherwise approvable: “Precursors to Diabetes in Japanese American Youth" as a Case Study', Kennedy Institute of Ethics Journal, Vol. 14, No. 2, pp. 165-86.

Russell, M.L., Moralejo, D.G. and Burgess, E.D. (2000) 'Paying research subjects: Participants' perspectives', Journal of Medical Ethics, Vol. 26, No. 2, pp. 126-30.

Ryan, M.A. (2006) 'The Politics of Risk: A Human Rights Paradigm for Children's Environmental Health Research', Environmental Health Perspectives, Vol. 114, No. 10, pp. 1613-16. 
Sanci, L.A., Sawyer, S.M., Weller, P.J., Bond, L.M. and Patton, G.C. (2004) 'Youth health research ethics: Time for a matureminor clause?', Medical Journal of Australia, Vol. 180, pp. 336-38.

Santelli, J., Geller, G., Chen, D., Speers, M., Botkin, J. and Laswell, S. (2005) 'Recruitment of Pregnant, Minor Adolescents and Minor Adolescents at Risk of Pregnancy into Longitudinal, Observational Research: The Case of the National Children's Study'. In: E. Kodish (ed.), Ethics and Research with Children: A Case-based Approach. New York: 0xford University Press, pp. 100-19.

Santelli, J. and Smith Rogers, A. (2002) 'Parental permission, passive consent, and "children" in research', Journal of Adolescent Health, Vol. 31, No. 4, pp. 303-04.

Santelli, J.S., Rosenfeld, W.D., DuRant, R.H., Dubler, N., Morreale, M., English, A. and Rogers, A.S. (1995) 'Guidelines for Adolescent Health Research: A Position Paper of the Society for Adolescent Medicine', Journal of Adolescent Health, Vol. 17, No. 5, pp. 270-76.

Santelli, J.S., Smith Rogers, A., Rosenfeld, W.D., DuRant, R.H., Dubler, N., Morreale, M., English, A., Lyss, S., Wimberly, Y. and Schissel, A. (2003) 'Guidelines for Adolescent Health Research: A Position Paper of the Society for Adolescent Medicine', Journal of Adolescent Health, Vol. 33, No. 5, pp. 396-409.

Saver, R.S. (2005) 'What IRBs could learn from Corporate Boards', IRB: Ethics \& Human Research, Vol. 27, No. 5, pp. 1-6.

Scarr, S. (1994) 'Ethical Problems in Research on Risky Behaviors and Risky Populations', Ethics \& Behavior, Vol. 4, No. 2, pp. 147-55.

Scherer, D.G. (1991) 'The capacities of minors to exercise voluntariness in medical treatment decisions', Law and Human Behavior, Vol. 15, No. 4, pp. 431-49.

Scherer, D.G., Brody, J.L., Annett, R.D., Hetter, J., Roberts, L.W. and Cofrin, K.M.W. (2005) 'Financial compensation to adolescents for participation in biomedical research: Adolescent and parent perspectives in seven studies', The Journal of Pediatrics, Vol. 146, No. 4, pp. 552-58.

Schlam, L. and Wood, J.P. (2000) 'Informed consent to the medical treatment of minors: Law and practice', Health Matrix, Vol. 10, No. 2, pp. 141-74.

Schuppli, C.A. and Fraser, D. (2007) 'Factors influencing the effectiveness of research ethics committees', Journal of Medical Ethics, Vol. 33, No. 5, pp. 294-301.

Scott-Jones, D. (1994) 'Ethical issues in reporting and referring in research with low-income minority children', Ethics \& Behavior, Vol. 4, No. 2, pp. 97-108.

Scott, D.A., Valery, P.C., Boyle, F.M. and Bain, C.J. (2002) 'Does research into sensitive areas do harm? Experiences of research participation after a child's diagnosis with Ewing's sarcoma', Medical Journal of Australia, Vol. 177, No. 9, pp. 507-10.

Scott, J.K., Wishart, J.G. and Bowyer, D.J. (2006) 'Do current consent and confidentiality requirements impede or enhance research with children with learning disabilities?', Disability \& Society, Vol. 21, No. 3, pp. 273-87.

Seiler, C.M., Kellmeyer, P., Kienle, P., Büchler, M.W., Knaebel, H.-P. and the INSECT Study Group (2007) 'Assessment of the ethical review process for non-pharmacological multicentre studies in Germany on the basis of a randomised surgical trial', Journal of Medical Ethics, Vol. 33, No. 2, pp. 113-18.

Sengupta, S. and Lo, B. (2003) 'The Roles and Experiences of Non-affiliated and Non-scientist Members of Institutional Review Boards', Academic Medicine, Vol. 78, No. 2, pp. 212-18.

Shah, S., Whittle, A., Wilfond, B., Gensler, G. and Wendler, D. (2004) 'How do Institutional Review Boards apply the federal risk and benefit standards for pediatric research?', Journal of the American Medical Association (JAMA), Vol. 291, No. 4, pp. 476-82.

Shamoo, A.E. (2002) 'Ethically Questionable Research with Children: The Kennedy Krieger Lead Abatement Study', Accountability in Research, Vol. 9, No. 3, pp. 167-75.

Shamoo, A.E. and Moreno, J.D. (2004) 'Ethics of research involving mandatory drug testing of high school athletes in Oregon', The American Journal of Bioethics, Vol. 4, No. 1, pp. 25-31.

Shamoo, A.E. and Woeckner, E. (2006) 'Research Ethics Boards: No data on quality of For-Profit or Non-Profit IRBs', PLoS Medicine, Vol. 3, No. 10, e459.

Sharp, R.R. (2002) 'Teaching old dogs new tricks: Continuing education in research ethics', The American Journal of Bioethics, Vol. 2, No. 4, pp. 55-56.

Sharp, R.R. and Quigley, R.B. (2003) 'Knowing who you want to be when you grow up: Implications for Pediatric Assent', The American Journal of Bioethics, Vol. 3, No. 4, pp. 14-15.

Sheard, L., Tompkins, C.N.E., Wright, N.M.J. and Adams, C.E. (2006) 'Non-commercial clinical trials of a medicinal product: Can they survive the current process of research approvals in the UK?', Journal of Medical Ethics, Vol. 32, No. 7, pp. 430-34.

Shore, N. (2007) 'Community-based Participatory Research and the Ethics Review Process', Journal of Empirical Research on Human Research Ethics, Vol. 2, No. 1, pp. 31-41.

Sieber, J.E. (1991) 'Openness in the Social Sciences: Sharing Data', Ethics \& Behavior, Vol. 1, No. 2, pp. 69-86.

Sieber, J.E. (1994) 'Issues presented by Mandatory Reporting Requirements to Researchers of Child Abuse and Neglect', Ethics \& Behavior, Vol. 4, No. 1, pp. 1-22.

Silberner, J. (1998) 'Remodeling IRBs', The Hastings Center Report, Vol. 28, No. 4, p. 5.

Silverman, D. (1993) Interpreting Qualitative Data: Methods for analyzing talk, text and interaction. London: Sage Publications.

Simon, C., Eder, M., Kodish, E. and Siminoff, L. (2006) 'Altruistic Discourse in the Informed Consent Process for Childhood Cancer Clinical Trials', The American Journal of Bioethics, Vol. 6, No. 5, pp. 40-47.

Simon, C.M. and Kodish, E. (2005) "Step into My Zapatos, Doc": Understanding and reducing communication disparities in the multicultural informed consent setting', Perspectives in Biology and Medicine, Vol. 48, Supplement 1, pp. s123-38.

Slaven, M.J. (2007) 'First Impressions: The Experiences of a Community Member on a Research Ethics Committee', IRB: Ethics \& Human Research, Vol. 29, No. 3, pp. 17-19. 
Smith, R., Monagham, M. and Broad, B. (2002) 'Involving Young People as Co-Researchers: Facing up to the Methodological Issues', Qualitative Social Work, Vol. 1, No. 2, pp. 191-207.

Sobel, R. (2007) 'The HIPAA Paradox: The Privacy Rule That's Not', The Hastings Center Report, Vol. 37, No. 4, pp. 40-50.

Solbakk, J.H. (1991) 'Ethics Review Committees [in biomedical research] in the Nordic countries: History, organization and assignments', HEC Forum, Vol. 3, No. 4, pp. 215-20.

Solyom, A.E. and Moreno, J.D. (2005) 'Protection of Children and Adolescents in Psychiatric Research: An Unfinished Business', HEC Forum, Vol. 17, No. 3, pp. 210-26.

Specker, B.L. and Saenger, E.L. (1994) 'Does radiation research in healthy children pose greater than minimal risk?', IRB: Ethics and Human Research, Vol. 16, No. 5, pp. 5-10.

Speckman, J.L., Byrne, M.M., Gerson, J., Getz, K., Wangsmo, G., Muse, C.T., Sugarman, J. and Consortium to Examine Clinical Research Ethics (2007) 'Determining the Costs of Institutional Review Boards', IRB: Ethics \& Human Research, Vol. 29, No. 2, pp. 7-13.

Spielman, B. (2003) 'Should Consensus be "The Commission Method" in the US? The Perspective of the Federal Advisory Committee Act, Regulations and Case Law', Bioethics, Vol. 17, No. 4, pp. 341-56.

Spriggs, M. (2004) 'Canaries in the mines: Children, risk, non-therapeutic research and justice', Journal of Medical Ethics, Vol. 30, No. 2, pp. 176-81.

Spriggs, M. (2007) 'When "Risk" and "Benefit" are open to interpretation - as is generally the case', American Journal of Bioethics, Vol. 7, No. 3, pp. 17-19.

SSHWC (2006) Qualitative Research in the Context of the TCPS: A Follow-up to the Giving Voice to the Spectrum Report and a Discussion Paper, from the Social Sciences and Humanities Research Ethics Special Working Committee to the Interagency Advisory Panel on Research Ethics. Ottawa, Canada: Interagency Advisory Panel and Secretariat on Research Ethics.

SSHWC (2007a) Continuing the Dialogue on Privacy and Confidentiality: Feedback and Recommendations arising from SSHWC's Recent Consultation, from the Social Sciences and Humanities Research Ethics Special Working Committee to the Interagency Advisory Panel on Research Ethics. Ottawa, Canada: Interagency Advisory Panel and Secretariat on Research Ethics.

SSHWC (2007b) Extending the Spectrum: The TCPS and Research involving Creative Practice: A Report with Questions for Furthering the Dialogue, from the Social Sciences and Humanities Research Ethics Special Working Committee to the Interagency Advisory Panel on Research Ethics. Ottawa, Canada: Interagency Advisory Panel and Secretariat on Research Ethics.

Stead, M., Eadie, D., Gordon, D. and Angus, K. (2005) ' “Hello, hello - it's English I speak!": A qualitative exploration of patients' understanding of the science of clinical trials', Journal of Medical Ethics, Vol. 31, No. 11, pp. 664-69.

Steven, G.G. (2006) 'Supplementing the Traditional Institutional Review Board with an Environmental Health and Community Review Board', Environmental Health Perspectives, Vol. 114, No. 10, pp. 1626-29.

Stevenson, C. and Beech, I. (1998) 'Playing the power game for qualitative researchers: The possibility of a post-modern approach', Journal of Advanced Nursing, Vol. 27, No. 4, pp. 790-97.

Stopp, G.H., Jr. (1985) 'The Internal IRB Structure: Models in Academic Settings', IRB: Ethics and Human Research, Vol. 7, No. 6, p. 9.

Strike, K.A., Anderson, M.S., Curren, R., van Geel, T., Pritchard, I. and Robertson, E. (2002) Ethical Standards of the American Educational Research Association: Cases and Commentary. Washington, DC: American Educational Research Association.

Sugarman, J., McCrory, D.C., Powell, D., Krasny, A., Adams, B., Ball, E. and Cassell, C. (1999) 'Empirical Research on Informed Consent', The Hastings Center Report, Vol. 29, No. 1, pp. S1-S42.

Sultana, F. (2007) 'Reflexivity, Positionality and Participatory Ethics: Negotiating fieldwork dilemmas in international research', ACME: An International E-Journal for Critical Geographies, Vol. 6, No. 3, pp. 374-85.

Susman, E.J., Dorn, L.D. and Fletcher, J.C. (1992) 'Participation in biomedical research: The consent process as viewed by children, adolescents, young adults and physicians', The Journal of Pediatrics, Vol. 121, No. 4, pp. 547-52.

Sweet, D.G. and Halliday, H.L. (2004) 'Applications to a research ethics committee', Current Paediatrics, Vol. 14, No. 6, pp. 495-500.

Tait, A., Voepel-Lewis, T. and Malviya, S. (2003a) ‘Do They Understand? Part II: Assent of children participating in clinical anesthesia and surgery research', Anesthesiology, Vol. 98, No. 3, pp. 609-14.

Tait, A., Voepel-Lewis, T. and Malviya, S. (2003b) 'Do They Understand? Part I: Parental consent for children participating in clinical anesthesia and surgery research', Anesthesiology, Vol. 98, No. 3, pp. 603-08.

Tait, A., Voepel-Lewis, T. and Malviya, S. (2007) 'Presenting Research Information to Children: A Tale of Two Methods', Anesthesia \& Analgesia, Vol. 105, No. 2, pp. 358-64.

Tait, A.R., Voepel-Lewis, T., Malviya, S. and Philipson, S.J. (2005) 'Improving the Readability and Processability of a Pediatric Informed Consent Document: Effects on parents' understanding', Archives of Pediatrics \& Adolescent Medicine, Vol. 159, No. 4, pp. 347-52.

Tandy, M.K. (1996) 'Independent Commercial IRBs', IRB: Ethics and Human Research, Vol. 18, No. 3, p. 10.

Taube, D.0. and Burkhardt, S. (1997) 'Ethical and Legal Risks associated with Archival Research', Ethics \& Behavior, Vol. 7, No. 1 , pp. 59-67.

Tauer, C.A. (2002) 'Central Ethical Dilemmas in Research involving Children', Accountability in Research, Vol. 9, No. 3, pp. 127-42.

Tayler, C., Farrell, A., Tennent, L. and Patterson, C. (2005) 'Researching communities: Towards beneficence'. In: A. Farrell (ed.), Ethical Research with Children. New York: Open University Press, pp. 138-49.

Taylor, H.A. (2003) 'Children under age 14 deserve more', The American Journal of Bioethics, Vol. 3, No. 4, pp. 33-34.

Taylor, H.A. (2007a) 'Instead of revising half the story, why not rewrite the whole thing?', American Journal of Bioethics, Vol. 7, No. 3, pp. 19-21. 
Taylor, H.A. (2007b) 'Moving Beyond Compliance: Measuring ethical quality to enhance the oversight of human subjects research', IRB: Ethics \& Human Research, Vol. 29, No. 5, pp. 9-14.

Thomas, N. and 0'Kane, C. (1998) 'The ethics of participatory research with children', Children \& Society, Vol. 12, No. 5, pp. 336-48.

Thompson, R.A. (1990) 'Behavioral Research involving Children: A Developmental Perspective on Risk', IRB: Ethics and Human Research, Vol. 12, №. 2, pp. 1-6.

Thompson, S. (1996) 'Paying respondents and informants', Social Research Update, Vol. 14 (Autumn).

Tickle, L. (2001) 'Opening Windows, Closing Doors: Ethical Dilemmas in Educational Action Research', Journal of Philosophy of Education, Vol. 35, No. 3, pp. 345-59.

Tilley, S. and Gormley, L. (2007) 'Canadian University Ethics Review: Cultural complications translating principles into practice', Qualitative Inquiry, Vol. 13, No. 3, pp. 368-87.

Tod, A.M., Nicolson, P. and Allmark, P. (2002) 'Ethical review of health service research in the UK: Implications for nursing', Journal of Advanced Nursing, Vol. 40, No. 4, pp. 379-86.

Tolich, M. and Fitzgerald, M.H. (2006) 'If ethics committees were designed for ethnography', Journal of Empirical Research on Human Research Ethics, Vol. 1, No. 2, pp. 71-78.

Toner, K. and Schwartz, R. (2003) 'Why a teenager over age 14 should be able to consent, rather than merely assent, to participation as a human subject of research', The American Journal of Bioethics, Vol. 3, No. 4, pp. 38-40.

Tu, J.V., Willison, D.J., Silver, F.L., Fang, J., Richards, J.A., Laupacis, A. and Kapral, M.K. (2004) 'Impracticability of Informed Consent in the Registry of the Canadian Stroke Network', The New England Journal of Medicine, Vol. 350, No. 14 , pp. 1414-21.

Underwood, M.K. (2005) ‘Observing Anger and Aggression among Preadolescent Girls and Boys: Ethical Dilemmas and Practical Solutions', Ethics \& Behavior, Vol. 15, No. 3, pp. 235-45.

Valentine, G. (1999) 'Being Seen and Heard? The Ethical Complexities of Working with Children and Young People at Home and at School', Ethics, Place \& Environment, Vol. 2, No. 2, pp. 141-55.

Valentine, G., Butler, R. and Skelton, T. (2001) 'The Ethical and Methodological Complexities of doing research with "Vulnerable" Young People, 'Ethics, Place \& Environment, Vol. 4, No. 2, pp. 119-25.

Van den Hoonaard, W.C (2006a) 'New Angles and Tangles in the Ethics Review of Research', Journal of Academic Ethics, Vol. 4, No. 1-4, pp. 261-74.

Van den Hoonaard, W.C. (2006b) 'Trends in Canadian Sociology. Master's Thesis in relation to Research Ethics Review, 1995-2004', Journal of Empirical Research on Human Research Ethics, Vol. 1, No. 4, pp. 77-88.

Van den Hoonaard, W.C. (2003) 'Is Anonymity an Artifact in Ethnographic Research?', Journal of Academic Ethics, Vol. 1, No. 2, pp. 141-51.

VandenBosch, T.M., Ward, B.G. and Mattison, D. (1999) 'A Reappraisal of Female Adolescent Participation in Drug Clinical Trials', IRB: Ethics and Human Research, Vol. 21, No. 1, pp. 1-5.

Wagener, D.K., Sporer, A.K., Simmerling, M., Flome, J.L., An, C. and Curry, S.J. (2004) 'Human Participants Challenges in Youth-Focused Research: Perspectives and Practices of IRB Administrators', Ethics \& Behavior, Vol. 14, No. 4, pp. 335-49.

Wald, D.S. (2004) 'Bureaucracy of ethics applications', British Medical Journal, Vol. 329, No. 7460, pp. 282-84.

Walsh, K. (2005) 'Researching sensitive issues'. In: A. Farrell (ed.), Ethical Research with Children. New York: Open University Press, pp. 68-80.

Ward, H.J.T., Cousens, S.N., Smith-Bathgate, B., Leitch, M., Everington, D., Will, R.G. and Smith, P.G. (2004) '0bstacles to conducting epidemiological research in the UK general population', British Medical Journal, Vol. 329, No. 7460, pp. 277-79.

Weed, M. (2004) 'Ethics, regulation, and biomedical research', Kennedy Institute of Ethics Journal, Vol. 14, No. 4, pp. 361-68. Weir, R.F. and Peter, C. (1997) 'Affirming the decisions adolescents make about life and death', The Hastings Center Report, Vol. 27, No. 6, pp. 29-40.

Weise, K.L., Smith, M.L., Maschke, K.J. and Copeland, H.L. (2002) 'National practices regarding payment to research subjects for participating in pediatric research', Pediatrics, Vol. 110, No. 3, pp. 577-82.

Weithorn, L.A. (1983) 'Children's capacities to decide about participation in research', IRB: Ethics and Human Research, Vol. 5, No. 2, pp. 1-5.

Weithorn, L.A. and Campbell, S.B. (1982) 'The competency of children and adolescents to make informed treatment decisions', Child Development, Vol. 53, No. 6, pp. 1589-98.

Wells, R.J., Gartside, P.S. and McHenry, C.L. (2000) 'Ethical issues arising when interim data in clinical trials is restricted to independent data monitoring committees', IRB: Ethics and Human Research, Vol. 22, No. 1, pp. 7-11.

Wendler, D. (2004) 'Risk Standards for Pediatric Research: Rethinking the Grimes Ruling', Kennedy Institute of Ethics Journal, Vol. 14, No. 2, pp. 187-98.

Wendler, D., Belsky, L., Thompson, K.M. and Emanuel, E.J. (2005) 'Quantifying the Federal Minimal Risk Standard: Implications for Pediatric Research without a prospect of direct benefit', Journal of the American Medical Association (JAMA), Vol. 294, No. 7, pp. 826-32.

Wendler, D. and Jenkins, T. (2008) 'Children's and their parents' views on facing research risks for the benefit of others', Archives of Pediatrics \& Adolescent Medicine, Vol. 162, No. 1, pp. 9-14.

Wendler, D. and Shah, S. (2003) 'Should children decide whether they are enrolled in non-beneficial research?', The American Journal of Bioethics, Vol. 3, No. 4, pp. 1-7.

Wendler, D., Shah, S., Whittle, A. and Wilfond, B.S. (2003) 'Non-beneficial research with individuals who cannot consent: Is it ethically better to enroll healthy or affected individuals?', IRB: Ethics and Human Research, Vol. 25, No. 4, pp. 1-4.

Whiteman, D.C., Webb, P.M., Purdie, D.M. and Green, A.C. (2003) 'National ethics committee urgently needed', Medical Journal of Australia, Vol. 178, No. 4, p. 187. 
WHO (2000) Operational Guidelines for Ethics Committees that review Biomedical Research. Geneva: World Health Organization.

Wiles, R., Crow, G., Heath, S. and Charles, V. (2006) Anonymity and Confidentiality, NCRM Working Paper Series 2/06. Southhampton: National Centre for Research Methods, Economic and Social Research Council. Available at: www.ncrm.ac.uk/ research/outputs/publications/WorkingPapers/2006/0206_anonymity_and_confidentiality.pdf (accessed February 2010)

Wiles, R., Heath, S., Crow, G. and Charles, V. (2005) Informed Consent in Social Research: A Literature Review. Southhampton: National Centre for Research Methods, Economic and Social Research Council.

Williamson, E., Goodenough, T., Kent, J. and Ashcroft, R. (2005) 'Conducting research with children: The limits of confidentiality and child protection protocols', Children \& Society, Vol. 19, No. 5, pp. 397-409.

Williamson, G.R. and Prosser, S. (2002) 'Action Research: Politics, ethics and participation', Journal of Advanced Nursing, Vol. 40, pp. 587-93.

Wilson, A., Grimshaw, G., Baker, R. and Thompson, J. (1999) ‘Differentiating between audit and research: Postal survey of health authorities' views', British Medical Journal, Vol. 319, No. 7219, p. 1235.

Wilson, M. (2005) 'Vulnerable Subjects and Canadian Research Governance', IRB: Ethics \& Human Research, Vol. 27, No. 6, pp. 9-11.

Wolf, L.E. and Lo, B. (2004) 'Untapped Potential: IRB Guidance for the Ethical Research Use of Stored Biological Materials', IRB: Ethics \& Human Research, Vol. 26, No. 4, pp. 1-8.

Wolf, L.E. and Zandecki, J. (2007) 'Conflicts of Interest in Research: How IRBs address their own conflicts', IRB: Ethics \& Human Research, Vol. 29, No. 1, pp. 6-12.

Wolthers, 0.D. (2006) 'A questionnaire on factors influencing children's assent and dissent to non-therapeutic research', Journal of Medical Ethics, Vol. 32, No. 5, pp. 292-97.

Woods, K. and McNamara, J.R. (1980) 'Confidentiality: Its effect on interviewee behavior', Professional Psychology, Vol. 11, No. 5, pp. 714-21.

Yan, E.G. and Munir, K.M. (2004) 'Regulatory and ethical principles in research involving children and individuals with developmental disabilities', Ethics \& Behavior, Vol. 14, No. 1, pp. 31-49.

Yee, W.C. and Andrews, J. (2006) 'Professional researcher or a "good guest"? Ethical dilemmas involved in researching children and families in the home setting', Educational Review, Vol. 58, No. 4, pp. 397-413.

Zywicki, T.J. (2007) 'Institutional Review Boards as Academic Bureaucracies: An Economic and Experiential Analysis', Northwestern University Law Review, Vol. 101, No. 2, pp. 861-96. 


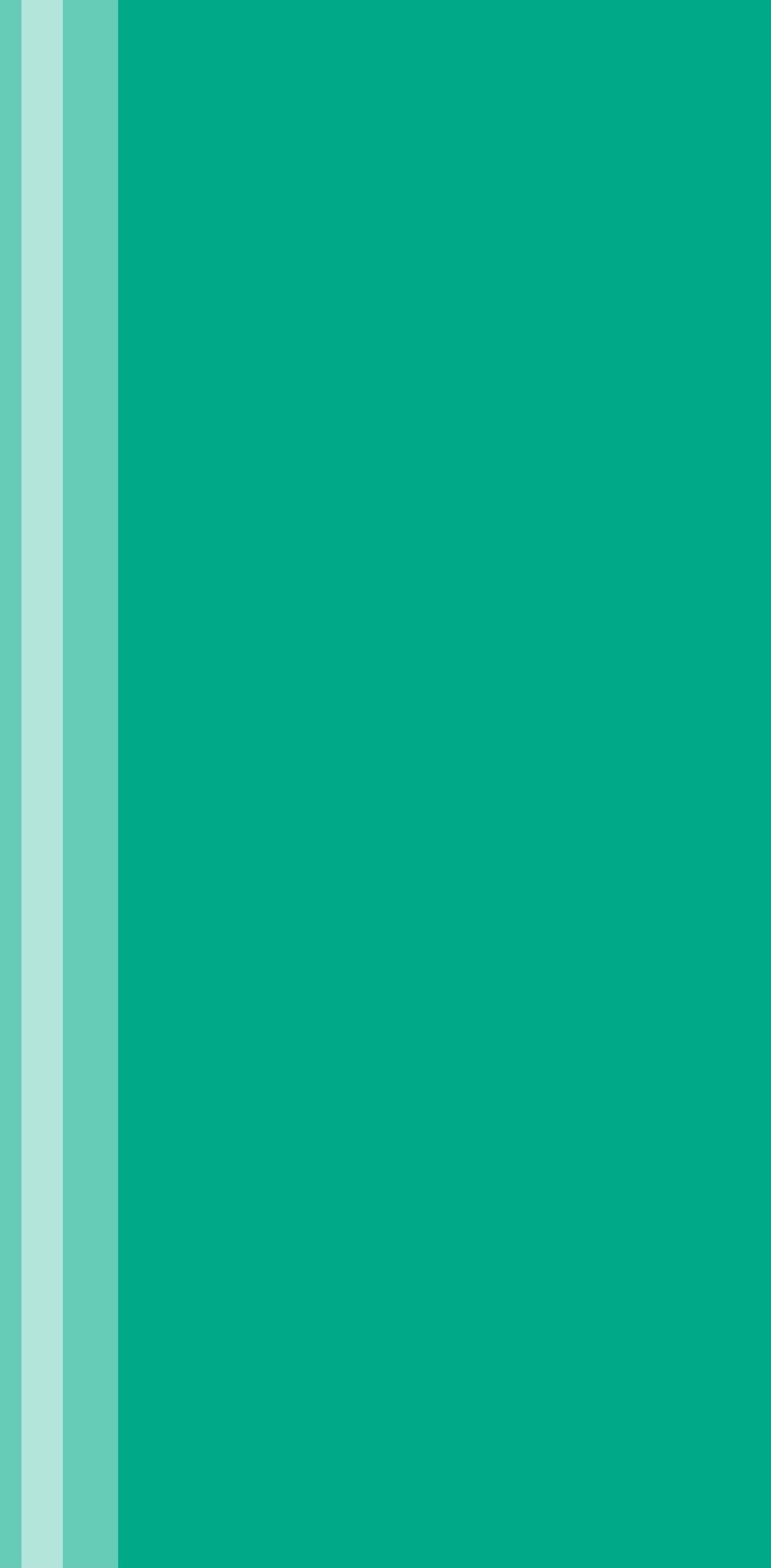


APPENDICIFS 


\section{Appendix 1: Survey Questionnaire Instrument}

\section{Research Ethics Committees Questionnaire}

\section{Review of REC Structures and Processes}

\section{GENERAL INFORMATION}

1. What is the official name and address of the Research Ethics Committee?

2. Contact details for secretary completing the questionnaire:

Name

Address

Telephone

Fax

3. Do you have a website? Yes $\square$ No $\square$

If Yes, please state website address

4. How long has this Research Ethics Committee been in operation?

years months

5. What resources are available to the REC? Please tick all that apply

a. Administrative personnel Yes $\square$ No $\square$

If Yes, on average, how many hours per week are dedicated to REC work by the administrative personnel?

b. $\quad$ A dedicated budget Yes $\square$ No $\square$

If Yes, please specify the amount

c. Database of REC activity Yes $\square$ No $\square$

d. Other (please specify)

\section{MEMBERSHIP OF REC}

6. How many members are on your committee?

7. Do you have a quorum to make decisions? Yes $\square$ No $\square$

8. If Yes, what number is your quorum?

9. How many times a year did the REC meet in 2006?

How long did the average meeting last? 
10. Please state the number of each of the following members on the committee whose primary function is:

Medical Doctor
Psychologist
Lay person
Statistician
Allied Health Professional

Scientist/Researcher

Ethicist

Nurse

Legal Professional

Religious Representative

Please state the role of any other members not included above

11. How long is the Chair's term of office?

12. How long is the Members' term of office?

\section{TRAINING}

13. Is training made available for REC members? Yes $\square$ No $\square$

If Yes, please give outline of specific training:

14. Is there a budget for training? Yes $\square$ № $\square$

\section{ACTIVITY LEVELS}

15. How many applications did the REC review in 2006?

If exact numbers are not available, please attempt an estimate (indicate estimate by *)

16. How many applications has the REC reviewed since its inception?

If exact numbers are not available, please attempt an estimate (indicate estimate by *)

17. How many applications did the REC approve in 2006?

18a. If you are part of the HSE, which disciplines/professions submitted proposals during 2006?

18b. If you are from an academic institution, please indicate what were the areas of research covered by these submissions? Tick all that are appropriate

Medicine and Health Sciences

Natural Sciences and Engineering

Psychology and Social Sciences

Education

Social Work

19. Approximately what percentage of submitted applications during 2006 involved children as research participants? 


\section{REC REMIT}

20. Please specify the types of research reviewed by the REC. Please tick all that apply

Quantitative Research

Qualitative Research

Clinical Audit/Evaluation Studies

Patient Satisfaction Surveys

21. Does your REC review clinical trials applications? Yes $\square$ No

If Yes, how many clinical trials did the REC review in 2006?

22. What percentage of the applications are for research being conducted to complete an MSc/PhD or other qualification?

23. Does your REC review applications for research that will be conducted on sites outside the location of the REC?

Yes $\square \quad$ No $\square \quad$ Unknown $\square$

24. Does the REC review applications from researchers outside of the organisation?

Yes $\square \quad$ No $\square$ Unknown $\square$

25. To your knowledge, do some researchers submit proposals to more than one REC?

Yes $\square \quad$ No $\square \quad$ Unknown $\square$

\section{APPLICATION PROCESS AND REVIEW PROCEDURES}

26. Does your REC have a standard application form? Yes $\square$ № $\square$

If Yes, can you attach a copy or include a weblink.

Can the application be submitted:

$\begin{array}{ll}\text { Online (through a website)? } & \text { Yes } \square \text { No } \square \\ \text { Via e-mail? } & \text { Yes } \square \text { No } \square \\ \text { Hard copy? } & \text { Yes } \square \text { No } \square\end{array}$

27. Does your REC have Standard Operating Procedures for approving applications?

Yes $\square \quad$ No $\square$

If Yes, can you attach a copy or include a weblink.

28. How is information on the REC review procedures made available to researchers? Please tick all that apply

Website

Intranet

Distribution of leaflets or brochures within the institution

Upon request by the researcher

Other (please specify)

Please attach any leaflets/documentation relating to REC information or relevant weblinks. 
29. Please indicate the average time in days between the submission of a proposal and the first feedback to the applicant? days

If exact numbers are not available, please attempt an estimate (indicate estimate by *)

30. Please indicate the average time in days between the submission of a proposal and a final decision? days

If exact numbers are not available, please attempt an estimate (indicate estimate by *)

31. How many times can the REC ask the applicant for supplemental information?

32. Are there alternative or expedited review procedures in place within your REC other than review by the full research ethics committee (e.g. Chairperson's approval, screening of applications, subcommittees)?

Yes $\square \quad$ No $\square \quad$ Unknown $\square$

If Yes, please give details

33. If an application is refused, does the REC provide an opportunity to appeal?

Yes $\square$ No $\square$

34. Does the REC monitor the research it has approved?

Yes $\square \quad$ No $\square \quad$ Unknown

Please specify

35. Does the ethics committee produce an annual report?

Yes $\square \quad$ No $\square$

If Yes, can you attach a copy or provide a weblink.

36. Do you, as secretary to the committee, have any further comments? 


\section{Appendix 2: List of Irish Research Ethics Committees for Human Subject Research}

* indicates that REC is authorised to review clinical trials according to the European Clinical Trials Directive.

** indicates that REC does not review research proposals, but is in charge of research ethics policy and governance for the institution.

\section{HOSPITALS AND HSE RESEARCH ETHICS COMMITTEES}

1. Beaumont Hospital Ethics (Medical Research) Committee* (www.beaumontethics.ie)

2. Bon Secours Research Ethics Sub-Committee

3. Cappagh National Orthopaedic Hospital Research Ethics Committee

4. Children's University Hospital, Temple Street Ethics Committee

5. Clinical Research Ethics Committee (CREC) of the Cork Teaching Hospitals (University College Cork)*

6. Coombe Women's Hospital Research Ethics Committee

7. Ethics Research Committee HSE Mid-Western Area*

8. Galway University Hospital Research Ethics Committee*

9. HSE Linn Dara CAMHS and Beechpark Autism Services Research Ethics Committee

10. HSE Midland Area Research Ethics Committee

11. HSE North Eastern Area Research Ethics Committee*

12. HSE South Eastern Area Research Ethics Committee*

13. James Connolly Memorial Hospital Research Ethics Committees

14. Letterkenny General Hospital Research Ethics Committee

15. Mater Misericordiae University Hospital and Mater Private Hospital Research Ethics Committee

16. Mayo General Hospital Research Ethics Committee

17. Naas General Hospital Research Ethics Committee

18. National Maternity Hospital Research Ethics Committee*

19. National Rehabilitation Hospital

20. Our Lady's Children's Hospital Research Ethics Committee*

21. The Rotunda Hospital Research Ethics Committee

22. Royal Victoria Eye \& Ear Hospital Research Ethics Committee

23. St. James's Hospital/AMNCH Research Ethics Committee*

24. St. Luke's Hospital Research Ethics Committee

25. St. Patrick's Hospital Research Ethics Committee

26. St. Vincent's Healthcare Group Ethics and Medical Research Committee*

27. St. Vincent's Hospital, Fairview Research Ethics Committee

28. Sligo General Hospital Research Ethics Committee*

29. Stewarts Hospital

\section{OTHER HEALTH AND DISABILITY CARE ORGANISATIONS}

30. Cheeverstown Research Ethics Committee

31. Children's Sunshine Home Research Ethics Committee

32. Cope Foundation

33. Daughters of Charity Research Ethics Committee

34. Enable Ireland Research Ethics Committee

35. KARE Research Ethics Committee

36. National Disability Authority (www.nda.ie/cntmgmtnew.nsf/researchhomepage?0penPage)

37. St. Francis Hospice Ethics Committee

38. St. John of God Hospitaller Services Research Ethics Committee

39. St. Michael's House Research Ethics Committee

40. Sisters of Charity Research Ethics Committee 


\section{ACADEMIC AND RESEARCH ORGANISATIONS}

41. Carlow Institute of Technology Research Ethics Committee (www.itcarlow.ie/downloads/Appendices/Appendix\%20E/Policy\%20\&\%20Procedures\%20on\%20 Ethics\%20in\%20Research.doc)

42. Dublin City University (DCU) Research Ethics Committee (www.dcu.ie/rss/research_ethics.shtml)

43. Dublin Institute of Technology (DIT) Research Ethics Committee (www.dit.ie/researchandenterprise/ethics)

44. Mater Dei Institute of Education Research Ethics Committee (independent of DCU)

45. NUI Galway Research Ethics Committee (www.nuigalway.ie/research/vp_research/ethics.html)

46. NUI Maynooth Research Ethics Committee (www.nuim.ie/researchsupport/research_ethics)

47. Royal College of Surgeons in Ireland (RCSI) Research Ethics Committee (www.rcsi-mub.com/index.jsp? $p=226 \& n=228$ )

48. St. Patrick's College Research Ethics Committee (independent of DCU) (www.spd.dcu.ie/main/research/InformationonEthicalResearch.shtml)

\section{TRINITY COLLEGE DUBLIN (TCD), Autonomous subcommittees}

49. Trinity College Dublin, Medical Faculty Research Ethics Committee (www.healthsciences.tcd.ie/committees/research_ethics_committee)

50. Trinity College Dublin, School of Nursing and Midwifery Research Ethics Advisory Committee (reports to TCD Medical Faculty Research Ethics Committee) (www.tcd.ie/Nursing_Midwifery/research/ethical_approval.php)

51. Trinity College Dublin, School of Psychology Research Ethics Committee (SPREC)

52. Trinity College Dublin, School of Social Work and Social Policy Research Ethics Committee (www.socialwork-socialpolicy.tcd.ie/research/ethics.php)

\section{UNIVERSITY COLLEGE CORK (UCC), Central governance committee and reporting subcommittees}

53. University College Cork, University Research Ethics Board (UREB)** (e-mail: ureb@ucc.ie) See also No. 5 above (Clinical Research Ethics Committee (CREC) of the Cork Teaching Hospitals)

54. University College Cork, Social Science Research Ethics Committee (SREC) (e-mail: srec@ucc.ie)

55. University College Cork, Applied Psychology Postgraduate Research Ethics Committee (www.ucc.ie/academic/apsych/ethics)

\section{UNIVERSITY COLLEGE DUBLIN (UCD), Central governance committee and reporting subcommittees}

56. University College Dublin, Research Ethics Committee (REC)** (www.ucd.ie/researchethics)

57. University College Dublin, Human Sciences Research Ethics Committee - Sciences (HREC-LS) (www.ucd.ie/researchethics/hrec_sciences.html)

58. University College Dublin, Human Sciences Research Ethics Committee - Humanities (HREC-HS) (www.ucd.ie/researchethics/hrec_humanities.html)

59. University College Dublin, School of Psychology Undergraduate Research Ethics Subcommittee (www.ucd.ie/researchethics/urec_spsy.html) 


\section{UNIVERSITY OF LIMERICK (UL), Central governance cum review committee and reporting subcommittees}

60. University of Limerick Research Ethics Committee (ULREC) (www2.ul.ie/web/WwW/Services/Research_Ethics)

61. University of Limerick, College of Education Research Ethics Committee (www2.ul.ie/web/WwW/Services/Research_Ethics)

62. University of Limerick, College of Humanities Research Ethics Committee (www2.ul.ie/web/WwW/Services/Research_Ethics)

63. University of Limerick, College of Informatics and Electronics Ethics Committee (CIEEC) (www2.ul.ie/web/WWW/Services/Research_Ethics)

64. University of Limerick, Kemmy Business School Ethics Committee (KBSREC) (www2.ul.ie/web/WWW/Services/Research_Ethics)

65. University of Limerick, Physical Education and Sports Sciences Research Ethics Committee (PESSREC)

(www2.ul.ie/web/WWW/Faculties/Education_\&_Health_Sciences/Departments/Physical_ Education_and_Sport_Sciences/Research/PESS_Research_Ethics?did=362398407)

66. Waterford Institute of Technology (WIT) Research Ethics Committee (www.wit.ie/Research/Support/Step-by-Step-Guide/Documents/4EthicalApproval)

\section{PROFESSIONAL ORGANISATIONS}

67. Faculty of Public Health Research Ethics Committee of the Royal College of Physicians of Ireland (www.rcpi.ie/Faculties/Pages/FacultyofPublicHealth.aspx)

68. Irish College of General Practitioners Research Ethics Committee* (www.icgp.ie/go/research/research_ethics)

\section{OTHER ORGANISATIONS}

69. Health Research Board (HRB) Research Ethics Committee (for HRB-funded research proposals only)

70. Irish Prison Service Prisoner Based Research Ethics Committee

71. Travellers' Research and Ethics Working Group 


\section{Appendix 3: Semi-structured Interview Schedule for Chairperson/Discussion topics for Administrators}

\section{SEMI-STRUCTURED INTERVIEW SCHEDULE FOR CHAIRPERSONS \\ REC experience}

In your opinion, what are essential requirements for the successful work of a REC?

1. What is working particularly well in your own committee?

2. Are there aspects that have been working less well or that have been particularly challenging for your REC?

What are your thoughts on how these might be resolved?

Prompt: Has ... been a problem in your REC:

- Workload?

- Expertise/training of REC members?

- REC recruitment?

- Specific troubling ethical issues?

3. How do you understand the role of the REC in your organisation?

Prompt:

- Vis-à-vis researchers (and student researchers)

Prompt: How could researchers be supported with regard to the ethics review process?

- Vis-à-vis organisation

- Vis-à-vis research participants and others

4. Have you received requests regarding ethical issues other than the review of research proposals?

In your opinion, should the REC be involved in any way in addressing such ethical issues?

\section{Children's research}

5. What significance does the review of children's research have in the work of your committee?

- comparative difficulty

- range of reviewed proposals

6. What are the most common problems that the REC identifies in submitted applications of research involving children?

7. What are the most significant challenges in decision-making for the REC in the review of children's research?

8. How could the work of your committee be further supported with regard to the review of children's research (on a local or national level)? 


\section{REC system}

9. What changes would you suggest to the existing REC system in Ireland to support the work of RECs in this country?

Prompt:

- Would you like to see changes regarding the review of multi-centre studies?

- What are your views on the possibility of centralising ethics review and reducing the number of ethics committees?

- Would you think more extensive national guidelines on ethical issues would be helpful for the work of RECs?

- What are your views on introducing an external panel of experts that can be consulted in difficult cases?

10. What about the existing system, should it be left as it is?

\section{DISCUSSION TOPICS FOR ADMINISTRATORS OF RECs}

\section{REC experience}

- Range of administrator's duties/activities.

- What has been working well from an administrative perspective?

- Perceived challenges to REC work and potential improvements/solutions.

- Specific issues:

- workload;

- training;

- recruitment of REC members.

\section{REC systemic issues}

- Relation of your REC to other REC bodies/sub-groups in your organisation. 


\section{Appendix 4: Focus Group Schedule for REC Members}

How long have you been a member of the REC and what profession/discipline do you represent in the committee?

\section{REC experience}

1. In your opinion, what conditions need to be in place to facilitate fruitful REC work for committee members?

2. What challenges do REC members face in their work for the committee? What do you see as potential solutions?

\section{Children's research}

3. What has been your experience with the review of children's research? Any difficult issues that you have encountered in the review process?

4. What issues have you encountered with regard to the following issues? Were they difficult to decide on?

- Recruitment of children for research.

- Design of informed consent and assent procedures.

- Deciding on the right balance between risk and benefit (what level of risk/harm is $0 K$ ?).

\section{Needs and supports}

5. What would you see as the main needs of parents and children in the research process?

6. Any suggestions on what might be useful supports for researchers to facilitate ethical conduct of children's research?

7. Any suggestions on what might be useful supports for REC members to facilitate the ethical review of children's research? 


\section{Appendix 5: Focus Group Schedule for Researchers}

What is your area of research and how long have you been working in this organisation?

\section{REC experience}

1. What has been your experience with ethical review of research so far, both within and outside of this organisation?

2. What challenges have you faced in the review process?

What do you see as potential solutions to these kinds of challenges?

\section{Children's research}

3. What ethical issues have you encountered in the conduct of children's research? Were they difficult to find a solution to?

What were the most difficult issues that you have encountered?

Prompt:

- Recruitment of children for research.

- Design of informed consent and assent procedures.

- Deciding on the right balance between risk and benefit (what level of risk/harm is $0 K$ ?).

- Other issues?

\section{Needs and supports}

4. What would you see as the main needs of parents and children in the research process?

5. Any suggestions on what might be useful supports for researchers to facilitate ethical conduct of children's research?

6. Any suggestions on potential changes to the REC review process in Ireland to facilitate children's research? 


\section{Appendix 6: Focus Group Schedule for Parents}

\section{Motivation and experience}

1. Has your child ever been involved in research?

If 'Yes':

2. What were the reasons you allowed your child to participate in research?

3. What was your and your child's experience with participating in research so far?

If 'No':

4. How do you think that parents decide whether or not to allow their children to participate in research?

\section{Awareness of the ethical requirements and review process}

5. What do you think are important obligations that the researcher has towards parents and children that they wish to collect information from?

6. Are you aware of any procedures that researchers have to go through before being allowed to conduct research with children?

Can you think of anything that should be in place to make sure researchers conduct research with children responsibly?

\section{Informed consent}

7. What role do you feel the school has to play in allowing researchers to work with children?

Should it be via notes from school or some other method?

8. If you have been involved in the process, how did it work for you as a way of allowing researchers to ask children their questions/gain information from children?

\section{Risk and benefit}

9. Once children are allowed to participate in research, do you feel it is important that they will benefit directly from the results of the research?

10. Do you think that there are circumstances when it might be $0 \mathrm{~K}$ for researchers to ask about issues that might make children uncomfortable?

\section{Other concerns}

11. Are there other worries/concerns that you might have about the research process? Anything you feel researchers should do in regard to parents?

Would it be important for you as a parent to know the results of the research? 
Appendix 7: Consent form used as part of the process for engaging with children

\section{STUDENT CONSENT}

I agree to take part in a workshop on my ideas about research with young people.

The reason for this research has been explained to me and I know I can withdraw at any time.

Signed:

Class name:

Date: 


\section{Appendix 8: Conclusions of HSE Report}

The HSE report Review of Research Ethics Committees and Processes in Republic of Ireland (2008, pp. 67-69) stated in its conclusion the following 6 observations regarding current needs and capacities in the REC sector in Ireland and also provided 4 recommendations as a potential way forward for Irish Research Ethics Committees, especially those in the HSE (available at: www.hse.ie/ eng/services/Publications/corporate/etr/Review_of_Research_Ethics.pdf).

\section{OBSERVATIONS}

The need for increased support capacity for RECs. Such support includes a national resource unit nationally. At REC level there is a need for dedicated administrative support, access to training, and access to specialist knowledge, IT support systems, dedicated time for participation on RECs and dedicated budgets for RECs to support their training, administrative support and IT requirements.

Participants expressed a resounding desire for standardised processes across research ethics committees. Standardisation was requested in relation to having standard operating procedures for applications to RECs, turnaround times and guidelines on a range of topics such as consent with vulnerable populations, membership and competencies of committees.

Communication was raised as an issue of concern at all levels of the research review process. This includes the need for improved systems at local and national levels between all stakeholders.

The openness and enthusiasm of all stakeholders to be involved in this area and the desire to formalise a network to support the development of knowledge and expertise.

There is a need for high level leadership on issues such as governance, quality and accountability in a way that supports local REC structures and processes.

There is a commitment to optimising the research experience to improve the health of the population while minimising the burden on RECs, researchers and, in particular, the population.

\section{[RECOMMENDATIONS]}

The REC Review Group proposes that to promote best practice in research in the HSE and to ensure quality and safety for the public, these issues are progressed through the following recommendations:

\section{Responsibility for HSE RECS}

That the unified Education, Training and Research structure in the HSE take the lead in relation to Research Ethics Committees. This could be done in one of two ways:

(a) Establish a national resource unit with responsibility for supporting Research Ethics Committees

or

(b) Contract out the task of supporting Research Ethics Committees to an experienced organisation.

There is a need for the development of an operational plan outlining the need for appropriate resources. 
The key functions and roles that need to be attended to by HSE centrally are:

- The establishment of a governance structure and a quality improvement process for RECS.

- The development of guidelines and SOPs that would cover issues such as consent, relevant legislation, standard competencies required by committee members, membership of committees, turnaround times for research, continuous quality assurance and the types of research the committees should approve.

- The development of a communication plan.

- The development of standardised operating procedures and application forms for use by all RECs.

- The development of national guidelines on feedback, review and annual reports.

- The identification of training needs for REC members and the research community, and the development and co-ordination of training.

- The provision of a standard database to REC activity.

- The provision of a system of arbitration.

- The establishment of a thoroughly integrated strategy between academic and all other RECs.

- To establish a national website.

- The setting up of a Specialist Panel/database of experts for RECs, the functions of which are outlined in Recommendation 2 below.

\section{Specialist Panel/database of experts for RECs}

Building on the expertise of stakeholders who have contributed to the Review, it is proposed that a specialist panel/database of experts be established. Their role would include:

- Advise on the implementation of this review.

- Be available to existing RECs for expert advice.

- Review existing REC structures and advise on issues of number of committees, location, membership, workload, etc.

- Advise on the best way to manage ethical approval for multi-centre research.

- Advise on the balance between central and local management of REC applications so that the overall process is more streamlined and efficient.

\section{Resources}

To ensure quality and safety in relation to research ethics:

- RECs need to be adequately resourced to perform their function, in particular to ensure dedicated administrative support and a database to record activity.

- Resources also need to be made available for training and competence building for REC members.

- Resources are needed to implement the recommendations of this report.

\section{Communication Systems for RECs}

A Communication Plan needs to be developed to ensure necessary communications systems are in place to enable:

- Communication to and from the HSE centrally.

- Ease of access to RECs for researchers.

- Communication between local RECs and researchers, including information on what requires REC review, how to access RECs and their procedures, and feedback mechanisms on their research proposals.

- Communication between researchers and committees, to include update reports and progress reports on completion of the research to the REC and to participants.

- Networking and communication on good practice.

- Improved discussion and communication between Irish RECs in order to allow for broader reflection on and dissemination of good practice standards and shared learning. 



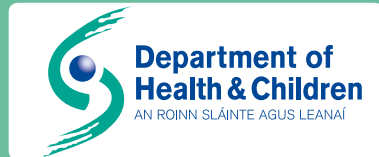

PUBLISHED BY THE STATIONERY OFFICE DUBLIN

To be purchased from the GOVERNMENT PUBLICATIONS SALES OFFICE, SUN ALLIANCE HOUSE, MOLESWORTH STREET, DUBLIN 2

or by mail order from

GOVERNMENT PUBLICATIONS,

POSTAL TRADE SECTION, 51 ST. STEPHEN'S GREEN, DUBLIN 2

Office of the Minister for Children and Youth Affairs Department of Health and Children

Hawkins House

Hawkins Street

Dublin 2

Tel: $+353(0) 16354000$

Fax: $+353(0) 16743223$

E-mail: omc@health.gov.ie

Web: www.omc.gov.ie

[Tel: 01647 6834; Fax: 01647 6843] Jor through any bookseller

Price: $€ 5.00$

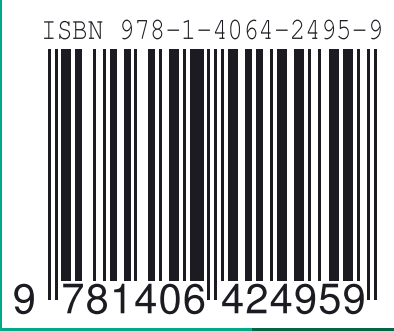

